José Leonel Arévalo García

\title{
Interference Mitigation Schemes for the Uplink of Massive MIMO in 5G Heterogeneous \\ Cellular Networks
}

Thesis presented to the Programa de Pós-Graduação em Engenharia Elétrica of the Departamento de Engenharia Elétrica do Centro Técnico Científico da PUC-Rio, as partial fulfillment of the requirements for the degree of Doutor em Engenharia Elétrica.

Advisor: Prof. Raimundo Sampaio Neto 


\section{José Leonel Arévalo García}

\section{Interference Mitigation Schemes for the Uplink of Massive MIMO in 5G Heterogeneous \\ Cellular Networks}

Thesis presented to the Programa de Pós-Graduação em Engenharia Elétrica of the Departamento de Engenharia Elétrica do Centro Técnico Científico da PUC-Rio, as partial fulfillment of the requirements for the degree of Doutor.

Prof. Raimundo Sampaio Neto

Advisor

Centro de Estudos em Telecomunicações - PUC-Rio

Prof. José Mauro Pedro Fortes

Centro de Estudos em Telecomunicações - PUC-Rio

Prof. Marcello Luis Rodrigues de Campos

UFRJ

Prof. Moisés Vidal Ribeiro

UFJF

Prof. Ernesto Leite Pinto

IME

Dr. César Augusto Medina Sotomayor

Centro de Estudos em Telecomunicações - PUC-Rio

Prof. Márcio da Silveira Carvalho

Coordinator of the Centro Técnico

Cientifico da PUC-Rio

Rio de Janeiro, March 28 ${ }^{\text {th }}, 2016$ 
All rights reserved.

\section{José Leonel Arévalo García}

The author received the Electrical Engineer degree from the National Autonomous University of Honduras - UNAH in 2008 (Tegucigalpa, Honduras), where he has also studied mathematics. After graduation he worked at the company NAVEGA (Tigo Business) dealing with telecommunications systems based on SDH, DWDM and Ethernet over SDH technologies. He received his Master degree in Telecommunications Systems from PUC-Rio in 2012 (Rio de Janeiro, Brazil). In the spring of 2015, during the course of his Ph.D. program, he went to Germany as a visiting researcher for the Communications Research Laboratory of the Ilmenau University of Technology.

Bibliographic data

Arévalo García, José Leonel

Interference mitigation schemes for the uplink of massive MIMO in 5G heterogeneous cellular networks / José Leonel Arévalo García ; advisor: Raimundo Sampaio Neto - 2016. 111 f. : il.(color.) ; $30 \mathrm{~cm}$

Tese (doutorado)-Pontifícia Universidade Católica do Rio de Janeiro, Departamento de Engenharia Elétrica, 2016.

Inclui bibliografia

1. Engenharia elétrica - Teses. 2. Sistemas MIMO multiusuário. 3. Detecção em múltiplos ramos com redução de reticulado e cancelamento sucessivo de interferências. 4 . Detecção por lista de tamanho variável. 5. Detecção desacoplada de sinais. 6. Redes celulares heterogêneas de quinta geração. I. Sampaio Neto, Raimundo. II. Pontifícia Universidade Católica do Rio de Janeiro. Departamento de Engenharia Elétrica. III. Título. 


\section{Acknowledgments}

I would like to express my sincere gratitude to my parents, Ercilia and Leoneth for having offered me unconditional support during the preparation of this thesis and for having taught me and cultivated the academic spirit throughout my life.

I would also like to express my gratitude to my love Sonia for her help and understanding in times of difficulty and to have brought to the world the greatest motivation of my life, the person who fills with happiness my heart and makes me smile even in difficult situations, my daughter María José.

Thanks to my sisters, Carolina and Mary and my brother Jorge, who have always been unconditionally supporting me.

I am very grateful to my colleagues of the Center for Studies in Telecommunications (CETUC), who have helped to love this place.

I wish also to thank CNPq and PUC-Rio, for the financial support, without which this work would not have been realized.

I am very grateful to Prof. Martin Haardt, who hosted me at Communication Research Laboratory of Ilmenau University of Technology for some months. The opportunity that he gave me to work with him and his team had a big impact on this thesis.

I would like to thank Prof. Rodrigo de Lamare, for supporting with important ideas and pragmatic suggestions the construction of this thesis and for helping me in learning how to make high level research. I appreciate the time that he dedicated to support this work.

I am extremely grateful to my adviser and friend, Prof. Raimundo Sampaio Neto for unconditional support and for following closely the evolution of this thesis. He gave me the freedom to choose the direction of my research which was very important to me. Finally, I would like to express my great admiration for Raimundo for his ethics and honesty as a professional and as a person. 


\section{Abstract}

Arévalo García, José Leonel; Sampaio Neto, Raimundo. Interference Mitigation Schemes for the Uplink of Massive MIMO in 5G Heterogeneous Cellular Networks. Rio de Janeiro, 2016. 111p. PhD Thesis - Departamento de Engenharia Elétrica, Pontifícia Universidade Católica do Rio de Janeiro.

In the first part of this thesis, we introduce two list detection schemes for the uplink scenario of multiuser multiple-input multiple-output (MUMIMO) systems. The proposed techniques employ a single lattice reduction (LR) transformation to modify the channel matrix between the users and the base station (BS). After the LR transformation, a reliable candidate for the transmitted signal vector, provided by successive interference cancellation (SIC) detection is obtained. In the proposed multi-branch lattice reduction SIC (MB-LR-SIC) detector, a fixed number of different orderings, generates a list of SIC detection candidates. The best candidate is chosen according to the maximum likelihood (ML) selection criterion. For the proposed variable list detection (VLD) scheme, an algorithm to decide if the current candidate has good quality or if it is necessary to further explore different orderings to improve the detection performance is employed. Simulation results indicate that the proposed schemes have a near-optimal performance while keeping its computational complexity well below that of the ML detector. An iterative detection and decoding (IDD) scheme based on the VLD algorithm is also developed, producing an excellent performance that approaches the single user (SU) scenario. In the second part of this thesis, a decoupled signal detection (DSD) technique which allows the separation of uplink signals, for each user class, at the base station (BS) for massive MIMO systems is proposed. The proposed DSD allows to implement the detection procedures proposed in the first part of this thesis in massive MIMO scenarios. A mathematical signal model for massive MIMO systems with centralized and distributed antennas in the future fifth generation $(5 \mathrm{G})$ heterogeneous cellular networks is also developed. A sum-rate analysis and a study of computational cost for DSD are also presented. Simulation results show excellent performance of the proposed DSD algorithm when combined with linear and SIC-based detectors.

\section{Keywords}

Multiuser multiple-input multiple-output systems; Multi-branch lattice reduction successive interference cancellation; Variable list detection; Decoupled signal detection; Fifth generation heterogeneous cellular networks. 


\section{Resumo}

Arévalo García, José Leonel; Sampaio Neto, Raimundo. Mitigação de Interferências em Sistemas MIMO Massivo Operando em Redes Heterogêneas de Quinta Geração (5G). Rio de Janeiro, 2016. 111p. Tese de Doutorado - Departamento de Engenharia Elétrica, Pontifícia Universidade Católica do Rio de Janeiro.

Na primeira parte desta tese, são desenvolvidos dois esquemas de detecção por listas para sistemas MIMO multiusuário. As técnicas propostas usam uma única transformação de redução de reticulado (LR) para modificar a matriz de canal entre os usuários e a estação base (BS). Após a transformação LR, um candidato confiável do sinal transmitido é obtido usando um detector de cancelamento sucessivo de interferências (SIC). No detector em múltiplos ramos com redução de reticulado e cancelamento sucessivo de interferências (MB-LR-SIC) proposto, um número fixo de diferentes ordenamentos para o detector SIC gera uma lista de possíveis candidatos para a informação transmitida. O melhor candidato é escolhido usando o critério maximum likelihood (ML). No detector por listas de tamanho variável (VLD) proposto, um algoritmo que decide se o candidato atual tem uma boa qualidade ou se é necessário continuar procurando por um candidato melhor nos ordenamentos restantes é utilizado. Os resultados numéricos mostram que os esquemas propostos têm um desempenho quase ótimo com uma complexidade computacional bem abaixo do detector ML. Um esquema de detecção e decodificação iterativa (IDD) baseado no algoritmo VLD é também desenvolvido, produzindo um desempenho próximo a um sistema mono usuário (SU) livre de interferências. Na segunda parte desta tese, uma técnica de detecção desacoplada de sinais (DSD) para sistemas MIMO massivo é proposta. Esta técnica permite que o sinal composto recebido na $\mathrm{BS}$ seja separado em sinais independentes, correspondentes a diferentes classes de usuários, viabilizando assim o uso dos procedimentos de detecção propostos na primeira parte desta tese em sistemas MIMO massivos. Um modelo de sinais para sistemas MIMO massivo com antenas centralizadas e/ou antenas distribuídas operando em redes heterogêneas de quinta geração é proposto. Uma análise baseada na soma das taxas e um estudo de custo computacional para DSD são apresentados. Os resultados numéricos ilustram o excelente compromisso desempenho versus complexidade obtido com a técnica DSD quando comparada com o esquema de detecção conjunta tradicional.

\section{Palavras-chave}

Sistemas MIMO multiusuário; Detecção em múltiplos ramos com redução de reticulado e cancelamento sucessivo de interferências; Detecção por lista de tamanho variável; Detecção desacoplada de sinais; Redes celulares heterogêneas de quinta geração. 


\section{Contents}

1 Introduction $\quad 12$

$\begin{array}{lll}1.1 & \text { Overview } & 12\end{array}$

$\begin{array}{lll}1.2 & \text { Motivation } & 13\end{array}$

$\begin{array}{lll}1.3 & \text { Contributions } & 15\end{array}$

$\begin{array}{ll}1.4 & \text { Thesis Outline } \\ 1.5 & \text { Notation }\end{array}$

$\begin{array}{ll}1.5 \text { Notation } & 18\end{array}$

$\begin{array}{lll}1.6 & \text { Acronyms } & 19\end{array}$

$\begin{array}{lll}1.7 & \text { Publication List } & 20\end{array}$

2 Fundamentals of MIMO Systems $\quad 22$

2.1 Overview 22

2.2 Multiuser MIMO System Model 22

2.3 MIMO Channel Capacity 23

2.4 Least Squares MIMO Channel Estimation 29

$\begin{array}{ll}2.5 & \text { Detection Techniques } \\ 2.6 & 29\end{array}$

2.6 MIMO Detection by List Generation 37

2.7 Numerical Results $\quad 41$

3 Lattice Reduction Detection for MIMO Systems 45

$\begin{array}{lll}3.1 & \text { Overview } & 45\end{array}$

3.2 Lattice Reduction Concept 46

3.3 Lattice Reduction Detection 49

3.4 Multi-Branch Lattice Reduction Successive Interference Cancellation Detection $\quad 52$

3.5 Variable List Detection $\quad 54$

3.6 Numerical Results $\quad 58$

4 Iterative Detection and Decoding for the Uplink of Multiuser MIMO $\begin{array}{ll}\text { Systems } & \mathbf{6 7}\end{array}$

$\begin{array}{lll}4.1 & \text { Overview } & 67\end{array}$

4.2 Iterative Detection and Decoding for Point-to-Point MIMO Systems 67

4.3 Iterative Variable List detection and Decoding $\quad 70$

$\begin{array}{lll}4.4 & \text { Numerical Results } & 73\end{array}$

5 Decoupled Signal Detection for the Uplink of Massive MIMO in Heterogeneous Cellular Networks $\quad 75$

$\begin{array}{lll}5.1 & \text { Overview } & 75\end{array}$

5.2 Proposed Massive MIMO Signal Model 75

5.3 Decoupled Signal Detection 78

5.4 Sum-Rate Analysis 83

5.5 Numerical Results $\quad 86$

6 Conclusions and Future Works $\quad 96$

$\begin{array}{lll}6.1 \text { Conclusions } & 96\end{array}$ 
$\begin{array}{lll}6.2 & \text { Future Work } & 98\end{array}$

$\begin{array}{lll}\text { A Appendix A } & 109\end{array}$

$\begin{array}{ll}\text { B Appendix B } & 110\end{array}$

$\begin{array}{lr}\text { C Appendix C } & 111\end{array}$

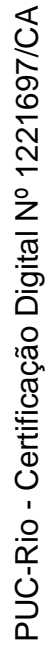




\section{List of Figures}

2.1 Multiuser MIMO system. 23

2.2 Decomposition of $\mathbf{H}$ when CSI is available at the transmitter side. 26

2.3 Tree diagram for Sphere Decoding 34

2.4 Successive Interference Cancellation Detector 35

2.5 Block diagram of the MB-SIC detector. 37

2.6 Block diagram of the MF-SIC detector. 39

2.7 The shaded area is the unreliable region for QPSK constellation 40

2.8 MIMO channel capacity when CSI is not available at the transmitter side.

2.9 BER vs SNR of SIC detectors with different detection ordering, QPSK modulation, $K=2$ users and $N_{t_{i}}=4$ transmit antennas per user.

2.10 BER vs SNR of existing MU-MIMO detectors with QPSK modulation, $K=3$ users and $N_{t_{i}}=2$ transmit antennas per user. All SIC detectors use column-norm-based ordering.

2.11 BER vs SNR of existing MU-MIMO detectors with QPSK modulation, $K=2$ users and $N_{t_{i}}=2$ transmit antennas per user. All SIC detectors use column-norm-based ordering. We also compared the performance with LS channel estimation (- -) and perfect channel estimation (-); $550 N_{t}$ symbols are transmitted, with 50 symbols used for training in the LS scheme.

2.12 BER vs SNR of existing MU-MIMO detectors with 16-QAM modulation, $K=4$ users and $N_{t_{i}}=1$ transmit antennas per user. All SIC detectors use column-norm-based ordering.

3.1 Proposed multi-branch lattice reduction successive interference cancellation detection block diagram.

3.2 Computational complexity of detection algorithms.

3.3 BER vs SNR performance for the proposed MB-LR-SIC and existing algorithms in a MU-MIMO scenario. All SIC detectors use a columnnorm-based ordering and QPSK modulation.

3.4 BER vs SNR performance for the proposed MB-LR- SIC and existing algorithms in a MU-MIMO scenario. All SIC detectors use a column-norm-based ordering and 16-QAM modulation.

40

3.5 BER vs SNR performance for the proposed VLD $\left(L_{\text {min }}=2, c_{1}=2.5\right)$ and existing algorithms with QPSK modulation in scenario $A$.

3.6 BER vs SNR performance for the proposed VLD $\left(L_{\text {min }}=2, c_{1}=2.5\right)$ and existing algorithms with QPSK modulation in scenario B $\left(L_{p}=0.7, \mu=3 \mathrm{~dB}, \rho_{t x}=\rho_{r x}=0.75\right)$.

3.7 BER vs SNR performance for the proposed $\operatorname{VLD}\left(L_{\text {min }}=2, c_{1}=5\right)$ and existing algorithms with QPSK modulation in scenario $A, L S$ channel estimation (- -) and perfect channel estimation (-). 
3.8 (a) BER vs SNR performance for the proposed VLD $\left(L_{\text {min }}=3, c_{1}=\right.$ 5) and existing algorithms with 16-QAM modulation in scenario A. (b) Average number of candidates tested by VLD vs SNR $\left(N_{t}=N_{r}=12\right)$.

3.9 (a) BER vs SNR performance for the proposed VLD $\left(L_{\min }=\right.$ $\left.3, c_{1}=40\right)$ and existing algorithms with 16-QAM modulation in scenario A. (b) Average number of candidates tested by VLD vs $\operatorname{SNR}\left(N_{t}=N_{r}=128\right)$.

4.1 Block diagram of a IDD transmission system over a Point-to-Point MIMO channel.

4.2 IDD receiver for MU-MIMO system.

4.3 BER vs SNR performance for MU-MIMO with IDD receiver, QPSK modulation and convolutional codes with $R_{c}=0.5$ in scenario $\mathrm{A}$.

4.4 BER vs SNR performance for MU-MIMO with IDD receiver, QPSK modulation and convolutional codes with $R_{c}=0.5$ in scenario $\mathrm{B}$ $\left(L_{p}=0.7, \mu=3 \mathrm{~dB}, \rho_{t_{x}}=\rho_{r_{x}}=0.7\right)$.

5.1 Heterogeneous Wireless Network.

5.2 (a) Sum Rate vs Number of Receive antennas. $N=4,\left|C_{n}\right|=8$ users per class, $N_{t_{k, n}}=1$ antennas per user. (b) Sum Rate vs Number of Receive antennas. $N=16,\left|C_{n}\right|=1$ users per class, $N_{t_{k, n}}=2$ antennas per user.

5.3 (a) Sum Rate vs Number of Receive antennas. $N=3,\left|C_{n}\right|=10$ users per class, $N_{t_{k, n}}=1$ antennas per user. (b) Sum Rate vs Number of Receive antennas. $N=2,\left|C_{n}\right|=16$ users per class, $N_{t_{k, n}}=1$ antennas per user.

Sum Rate vs SNR. N=8, $\left|C_{n}\right|=1$ users per class, $N_{t_{k, n}}=8$ antennas per user. We consider DAS configuration with $N_{B}=96$, $D=4$ and $Q=8$.

5.5 Computational complexity versus number of user class, $K=100$ active users, $N_{t_{k, n}}=2$ antennas per user, $N_{r}=200$ receive antennas. 90

5.6 Computational complexity versus number of active user, $N=5$ classes of user, $N_{t_{k, n}}=2$ antennas per user, $N_{t}=K N_{t_{k, n}}$ transmit antennas and $N_{r}=3 N_{t}$ receive antennas.

5.7 Computational complexity versus number of antennas per user, $K=10$ active user, $N=10$ classes of users, $N_{t}=K N_{t_{k, n}}$ transmit antennas and $N_{r}=2 N_{t}$ receive antennas.

5.8 BER versus SNR with $K=12$ active users, $N=3$ classes of users, $N_{t_{k, n}}=3$ transmit antennas per user and $N_{r}=36$ receive antennas.

5.9 BER versus SNR with $K=8$ active users, $N_{t_{k, n}}=8$ transmit antennas per user, $N=8$ classes of users and $N_{r}=128$ receive antennas.

5.10 BER versus SNR with $K=64$ active users, $N_{t_{k, n}}=1$ transmit antennas per user, $N=4$ classes of users, $N_{B}=34, D$ remote antennas arrays with $Q=7$ receive antennas per array. 


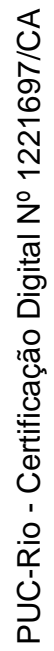

To María José. 


\section{Introduction}

\section{1}

\section{Overview}

Telecommunications systems play a fundamental role in the current globalized world in areas such as business, education, economics, science, healthcare, transportation and in our social and cultural life. Some technologies have been developed to help us to communicate with each other and to access information more easily at any time and anywhere. Practical electrical telecommunications systems started to appear with the telegraph in 1830s. The electrical telephone was presented in 1870s by Alexander Graham bell. In the late 1880s, Heinrich Hertz proved the prediction made in 1861 by James Clerk Maxwell, in the four laws about electromagnetism, by transmitting the first electromagnetic signal over a short distance, which represent the first antenna system. However, it was Nikola Tesla in 1892 who had the idea of using that technology to transmit signals from one place to another. In 1894 Guglielmo Marconi built the first complete and commercially successful wireless telegraph system based on airborne electromagnetic waves (radio transmission). The principle of the modern computer was first described by Alan Turing in 1936 . In 1979 the first automatic analog cellular system was deployed. Practical implementations of computer networks began in the late 1980s and over 1990s. In the 1990s, the World Wide Web was introduced. These inventions, among others, which began less than two hundred years ago, represent the basis of the technological revolution that we live in nowadays with an explosion of smart devices invading a big part of our lives and which have changed the way that we interact with other humans and machines. In that way, wireless communications systems represent an important part of the communication universe which is in constant evolution caused by the increasing requirements in term of data rates, latency and energy efficiency.

Mobile data traffic grew 65 percent between 2014 and 2015. The growth in data traffic is being driven both by increased mobile subscriptions and a continued increase in average data volume per subscription. Predictions published in [1]- [2] shows that the number of mobile broadband subscriptions is growing globally by around 25 percent per year. Smartphones make up the majority of mobile broadband devices today and subscriptions are expected to almost double by 2021. As the number of smart terminals and their emerging 
applications are growing, some research challenges need to be addressed for the future evolution of communications systems. Under this consideration, higher peak and user data rates, reduced latency, low energy consumption and low computational cost requirements for the signal processing will be necessary for the fifth generation $(5 \mathrm{G})$ of wireless communications systems, which are expected to be deployed commercially around 2020 [3]- [8]. In 5G networks, enhancements in mobile broadband services will be provided. For $5 \mathrm{G}$ peak data rates of 10 Gbps (Giga bit per second) for low mobility and 1 Gbps for high mobility are expected that represents an increase of 10 times in the data rates with respect to $4 \mathrm{G}[9]-[13]$.

\section{2}

\section{Motivation}

In recent years, the use of multiple transmit and receive antennas, a technique know as multiple-input multiple-output (MIMO) systems, are being considered as one of the most promising technologies. The use of MIMO is key to combating the fading and increasing the spectral efficiency, and to providing receive diversity gain and spatial multiplexing [14]- [15]. The channel capacity of MIMO systems increases with the minimum of transmit and receive antennas, which makes high data rates transmission possible [16]- [17]. Despite its advantages, MIMO suffers from interference between multiple antennas and, in multiuser scenarios, is affected by the multiuser interference.

Large-scale MIMO systems, also known as massive MIMO, are strong candidates for $5 \mathrm{G}$ cellular networks which use a large number of antennas to serve a high number of user terminals at the same time without requiring extra bandwidth resources [18]- [26]. This new greater scale version of traditional MIMO systems, where a restricted number of antenna elements is used, is designed to exploit the benefits of extra degrees of freedom obtained by the use of more antennas [27]. Massive MIMO can increase the spectral efficiency 10 times or more when compared with its predecessor [28]. In the Long Term Evolution (LTE) standard [9], which operates in the frequency-division duplex (FDD) mode, the users estimate the channel response and feed it back to the base station. For massive MIMO this might not be feasible, due to the large number of channel coefficients that each user needs to estimate being proportional to the number of antenna elements at the BS. In this thesis we focus on the uplink, the reason is that the most natural transmission mode to operate in massive MIMO is the time-division duplexing (TDD) mode, where a reciprocity between the uplink and downlink channels can be obtained, if we use appropriate calibration techniques to combat the distortions induced 
by hardware imperfections, since the base station can offer more processing resources aimed at estimating the channels between users' terminals and the BS. On the downlink it is possible to use different precoding schemes to mitigate the interference for the received signals at the mobile users. Such precoding schemes rely on the channels estimated on the uplink.

One of the main research challenges of massive MIMO is to develop computationally simple ways to process the large number of signals received at the BS. The interference between antennas and users, propagation effects such as correlation, path loss and shadowing, thermal noise and signal degradation due to the hardware imperfections need to be suppressed. In MIMO systems the maximum likelihood (ML) detector is able to provide optimal performance and full receive diversity gain. However, the ML detector may not be used in high dimensional systems due to its exponential computational complexity. Some near optimal ML schemes have been proposed as the generalized sphere decoding (GSD) [29]- [32], however the computational complexity at low signal-to-noise ratio (SNR) and large number of antennas is still high. Linear detection techniques such as maximum ratio combining (MRC), zero forcing (ZF) and minimum mean square error (MMSE) are good options in terms of computational complexity [33], however, due to the impact of interference and noise, linear detectors offer a limited performance, which is not compatible with the growing demand for high data rates. The performance of linear detectors can be improved without significant burden in the computational cost, using some nonlinear sub-optimal detector based on successive interference cancellation (SIC) [34]- [35], e.g., multibranch SIC (MB-SIC) [36]- [37] and multifeedback SIC (MF-SIC) [38]. However SIC-based detectors, despite having a better performance than linear detectors, still suffer from error propagation and performance degradation [39]- [40]. Recently, lattice reduction (LR) based detectors have been proposed to improve the performance while their computational cost does not increase much [41]- [51]. The LR technique introduces a preprocessing in the channel matrix, generating a nearly orthogonal basis in the same lattice, i.e., transform the channel matrix into a better conditioned channel matrix that allows one to mitigate the effects of interference and channel correlation. To compute the new equivalent channel matrix, the complex Lenstra, Lenstra and Lovàsz (CLLL) algorithm, which has a low computational complexity has recently been adopted [52]. In [53]- [54] LR has been shown to improve the performance of linear detectors. The LR-SIC detector has been developed in [55] offering a good performance with full receive diversity. In general, LR-based detectors have a full diversity gain with low computational complexity when compared with the ML detector, however a key problem with 
these detectors, is that the performance gap to the ML detector increases with the number of antennas, number of users and the modulation order. Hence, there is need to develop detection approaches that can obtain a more attractive trade-off between performance and complexity for high-dimensional systems.

The iterative detection and decoding (IDD) schemes can be used to improve the performance of coded MIMO systems where the maximum a posteriori probability (MAP) detection is desirable [56]. The implementation of the MAP detector is not viable due to its high computational cost. For this reason detection alternatives for coded systems should be investigated.

In the next generation of wireless communication systems [3], it is expected that a large number of users with different configurations and requirements is connected to the network. Therefore, it is necessary to design heterogeneous networks capable of interconnecting the different user types with each other [57]. The received signals from this large number of connected devices such as, metering equipment, sensors, environmental monitoring devices, health care gadgets, security management products, smart grid components, smart phones and tablets need to be separated in order to detect the transmitted information according to their different data requirements. In this context, distributed antenna systems (DAS) with massive MIMO are a promising alternative for the $5 \mathrm{G}$ cellular architectures [58]- [63], where the BS will be equipped with a large number of antennas and some remote antenna arrays or radio heads will be distributed around the cell and connected to the BS via optical fiber. The signals associated with different remote antenna arrays are processed at the BS. DAS have low path loss effects, improve the coverage and the spectral efficiency [64] [65]. The energy consumption of users is reduced and the transmission quality is improved due to the shorter distances between users and some remote antenna arrays. For this vision of $5 \mathrm{G}$ wireless networks, which includes a combination of massive MIMO, heterogeneous networks, and distributed antenna systems, efficient signal processing techniques at the BS are necessary.

\section{3}

\section{Contributions}

- In the first part of this thesis, we will focus in to propose two advanced interference mitigation schemes, for the uplink of MU-MIMO for cellular systems that allows a designer to reduce significantly the performance gap to the optimal detector.

- The first detector proposed in this work combines LR, SIC and MB schemes to devise an MB-LR-SIC detector. The main idea 
is to employ an LR transformation on the channel matrix, then generate a fixed number of branches, where each branch has a different ordering pattern and produces different symbol estimate vectors using the LR-SIC structure. An efficient implementation for the LR-SIC detector is proposed and evaluated. The branch with the best performance among the list of candidates is selected using the ML criterion. Despite the good performance presented by MBLR-SIC, the high computational complexity caused by the fixed number of candidates that need to be tested have motivated the second detection technique proposed in this thesis.

- The variable list detection (VLD) scheme proposed here computes a variable number of solution candidates for the transmitted signal vector in the LR domain updating the decision if a better candidate is found. The VLD scheme employs an LR transformation on the channel matrix, then generates a reliable candidate for the transmitted information vector in the LR domain. The ordering of LR-SIC symbol detections is implemented according to the MSE values of the estimated symbols in the LR domain. Based on the quality of the current candidate vector, a number of new candidates to be tested is determined. VLD adopts a stopping criterion that considers the ML cost of the best result so far as well as its first and second order moments for an error free decision. The numerical results show that the proposed VLD scheme has excellent performance and approaches the optimal ML detector. A computational complexity study and the benefits of the MSE ordering for the LR-SIC in VLD scheme are also examined in this thesis.

- An IDD algorithm for the VLD scheme is also developed which computes reliable soft estimates of the transmitted symbols for a convolutional coded system.

- The performance of the proposed detectors, with practical channel estimation algorithms is compared with several existing detectors in long term evolution advanced (LTE-A) and large-scale MIMO scenarios. We consider a realistic MU-MIMO scenario with path loss, log-normal shadowing and multiple antennas per user. An analysis of complexity for the proposed detection algorithms is also presented.

- The detectors proposed in the first part of this thesis still have a considerable computational cost for high-dimensional systems. If all 
signals from all active users are coupled in the detection process, the BS could spend unnecessary processing resources since some types of users might not require a very high performance. In the second part of this thesis, we propose an algorithm for the uplink of massive MIMO systems to separate the combined received signal of all users at the BS, into independent signals for each user class.

- The proposed decoupled signal detection (DSD) performs a decomposition into multiple independent single user class signals, where all users in a class have the same data requirements and a common complex modulation. Assuming that the channel state information (CSI) was previously estimated, DSD employs a common channel inversion and a $\mathrm{QR}$ decomposition to decouple the received signal. Applying the proposed DSD algorithm, the computational cost of the signal processing is reduced and it is possible to have flexibility on the detection procedure at the BS.

- A signal model for heterogeneous networks with different classes of users and an arbitrary configuration of centralized antenna systems (CAS) and distributed antenna systems (DAS) is also introduced.

- A sum rate analysis and a computational complexity study for the proposed DSD are presented. The performance of the proposed scheme is evaluated in a realistic scenario with propagation effects and compared with existing approaches.

\section{4 \\ Thesis Outline}

The remainder of this thesis is organized as follows:

- In Chapter 2, the basic concepts for MU-MIMO systems are studied, starting with the mathematical representation of MU-MIMO systems. Then, channel capacity aspects and a channel estimation technique for MIMO channels is described. A number of existing optimal and suboptimal MU-MIMO detection techniques are also presented.

- Chapter 3 introduces the lattice reduction concept and some detection schemes based on the LR transformation. The proposed MB-LR-SIC detector and the proposed VLD scheme, which obtains a near optimal performance with low computational complexity, are also introduced.

- In Chapter 4 , we propose an IDD receiver scheme which uses the proposed VLD algorithm to compute a reliable soft estimation of the transmitted information. 
- In Chapter 5, we propose a signal model for 5G heterogeneous cellular networks. We also propose the DSD algorithm that allows one to separate the users per classes and to make the detection procedures independently of other classes of users at the BS.

- Chapter 6 concludes this thesis by summarizing the benefits of the proposed detection schemes followed by a discussion of future work in detection for heterogeneous cellular networks within the $5 \mathrm{G}$ context.

\section{5}

\section{Notation}

$a$

a

A

$A_{i, j}$

$\mathbf{a}_{i}$

$\mathbf{A}^{-1}$

$\mathbf{A}^{T}$

$\mathbf{A}^{\mathcal{H}}$

$\mathbb{T}[\mathrm{A}]$

$\operatorname{det}(\mathbf{A})$

$\mathbb{E}[\cdot]$

$\operatorname{Re}(\cdot)$

$\operatorname{Im}(\cdot)$

$\langle\mathbf{a}, \mathbf{b}\rangle$

$\|\mathrm{a}\|$

$\log (\cdot)$

$\lceil a\rfloor$

$|a|$

$\mathbf{I}_{N}$

$\mathbb{Q}$

$\mathbb{Z}$
Scalar value

Complex-valued vector

Complex-valued matrix

Entry in $i$-th row and $j$-th column of $\mathbf{A}$

$i$-th column of $\mathbf{A}$

Inverse of $\mathbf{A}$

Transpose of A

Hermitian of $\mathbf{A}$

The trace operation of a square matrix $\mathbf{A}$

Determinant of matrix A

Expected value operator

Real part of a complex number

Imaginary part of a complex number

Inner product of $\mathbf{a}$ and $\mathbf{b}$

Norm of a

Natural logarithm

The nearest integer to $a$

Absolute value of $a$

Am $N \times N$ identity matrix

Set of complex numbers

Set of integer numbers 
1.6

Acronyms

$4 \mathrm{G}$

$5 \mathrm{G}$

APP

AWGN

BER

BPSK

BS

CAS

CLLL

CSI

DAS

$\mathrm{dB}$

DSD

FDD

FLOPs

Gbps

GSD

IDD

ISI

LLL

LLR

LR

LS

LTE

LTE-A

MAP

$\mathrm{MB}$

Mbps

$\mathrm{MF}$

MIMO

ML

MMSE

MRC

MS

pdf

PIC

QAM
Fourth Generation

Fifth Generation

A posteriori probability

Additive white Gaussian noise

Bit error rate

Binary phase shift keying

Base station

Centralized antennas systems

Complex-valued LLL

Channel state information

Distributed Antenna System

Decibel

Decoupled signal detection

Frequency-division duplex

Floating point operation

Giga bits per second

Generalized sphere decoding

Iterative detection and decoding

Intersymbol interference

Lenstra-Lenstra-LovA $\tilde{A}_{j z}$

Log-likelihood ratio

Lattice reduction

Least square

Long Term evolution

LTE advanced

Maximum a posteriori probability

Multiple branch

Mega bits per second

Multiple feedback

Multiple-input multiple-output

Maximum likelihood

Minimum square error

Maximum radio combining

Mobile station

Probability density function

Parallel interference cancellation

Quadrature amplitude modulation 


$\begin{array}{ll}\text { QPSK } & \text { Quadrature phase shift keying } \\ \text { SAC } & \text { Shadow area constraints } \\ \text { SC } & \text { Soft Cancellation } \\ \text { SD } & \text { Sphere decoding } \\ \text { SIC } & \text { Successive interference cancellation } \\ \text { SINR } & \text { Signal to interference plus noise ratio } \\ \text { SISO } & \text { Soft-input soft-output } \\ \text { SNR } & \text { Signal-to-noise ratio } \\ \text { TDD } & \text { Time-division duplex } \\ \text { VLD } & \text { Variable list detection } \\ \text { ZF } & \text { Zero Forcing }\end{array}$

\section{7 \\ Publication List}

\section{Journal Papers}

1 L. Arévalo, R. de Lamare, M. Haardt, and R. Sampaio-Neto, "Decoupled Signal Detection for the Uplink of Massive MIMO in Heterogeneous Networks," IEEE Transactions on Communications, under revision, 2016.

2 L. Arévalo, R. de Lamare, and R. Sampaio-Neto, "Variable List Detection for Multiuser MIMO Systems," IEEE Transactions on Vehicular Technology, under the second revision, 2016.

3 L. Arévalo, and R. Sampaio-Neto, "Decoupled Lattice Reduction Detection for Massive MIMO Sytems," IET Communications, under preparation, 2016.

4 D. M. Pereira, L. Arévalo, R. P. David, and R. Sampaio-Neto, "ChipSpread CDMA Transmission in Presence of Imperfections: A Comparative Analysis," IET Communications, under preparation, 2016.

\section{Conference Papers [66]- [70]}

1 L. Arévalo, R. de Lamare, M. Haardt, and R. Sampaio-Neto, "Uplink Block Diagonalization for Massive MIMO-OFDM Systems with Distributed Antennas," IEEE International Workshop on Computational Ad- 
vances in Multi-Sensor Adaptive Processing (CAMSAP), Cancun, Mexico, December 2015, pp. 389 - 392.

2 L. Arévalo, C. A. Medina, and R. Sampaio-Neto, "Playing with Blocks: A New Combination of Block Transmission and the CDMA Multiple Access Technique," 2015 IEEE 81st Vehicular Technology Conference (VTC Spring), Glasgow, Scotland, May 2015, pp. 1 - 4.

3 L. Arévalo, R. de Lamare, and R. Sampaio-Neto, "Iterative MultiBranch Lattice Reduction-Aided Successive Interference Cancellation for Multiuser MIMO Systems," 19th International ITG Workshop on Smart Antennas (WSA), Ilmenau, Germany, March 2015, pp. 1 - 5.

4 L. Arévalo, R. de Lamare, K. Zu, and R. Sampaio-Neto, "Multibranch lattice reduction successive interference cancellation detection for multiuser MIMO systems," IEEE International Symposium on Wireless Communications Systems (ISWCS), Barcelona, Spain, August 2014, pp. 219 -223 .

5 L. Arévalo, J. A. Apolinario, M. L. R. de Campos, and R. Sampaio-Neto, "Convex Combination of Three Affine Projections Adaptive Filters," IEEE International Symposium on Wireless Communications Systems (ISWCS), Ilmenau, Germany, August 2013, pp. 1 - 5. 


\section{2}

\section{Fundamentals of MIMO Systems}

\section{1}

\section{Overview}

MIMO systems offer a spatial multiplexing gain, i.e., data streams can be transmitted in parallel over the same channel [16]. This leads to substantially higher channel capacity and transmission rates. The use of multiple antennas offers diversity gain on systems that operate in fading channels [17]. The diversity order is equal to the number of independently received fading paths, which corresponds to the slope of the bit-error-rate (BER) curve.

In this chapter, the basic concepts of MIMO systems are studied.

This chapter is organized as follows. It is starting with the mathematical representation of MU-MIMO systems in Section 2.2. Then, the MIMO channel capacity and the least squares channel estimation technique for MIMO channels are described in Section 2.3 and Section 2.4, respectively. In Section 2.5, we also review some important existing detection techniques in the literature. Two detection techniques by list generation are then described in Section 2.6. To conclude, numerical results comparing the performance of the different detection techniques are presented in Section 2.7.

\section{2}

\section{Multiuser MIMO System Model}

To explore the multiuser diversity gain [71] of MU-MIMO systems [72], we consider the uplink channel scenario of a MU-MIMO system with $K$ active users, as depicted in Figure 2.1, the $k$-th mobile station (MS) transmits a data stream divided into $N_{t_{k}}$ sub-streams through $N_{t_{k}}$ antennas. The users send signals to one base station (BS) equipped with $N_{r}$ receive antennas, where $N_{r} \geqslant N_{t}=\sum_{k=1}^{K} N_{t_{k}}$ and $N_{t}$ is the total number of transmit antennas. The received signal vector at the $\mathrm{BS}$ is given by

$$
\begin{aligned}
\mathbf{y} & =\mathbf{H}_{1} \mathbf{s}_{1}+\mathbf{H}_{2} \mathbf{s}_{2}+\ldots+\mathbf{H}_{K} \mathbf{s}_{K}+\mathbf{n} \\
& =\sum_{k=1}^{K} \mathbf{H}_{k} \mathbf{s}_{k}+\mathbf{n},
\end{aligned}
$$

where $\mathbf{s}_{k}$ is the $N_{t_{k}} \times 1$ transmitted signal vector by the $k$-th user at one time slot taken from a common modulation constellation, denoted by $\mathcal{A}=$ $\left\{a_{1}, a_{2}, \ldots, a_{O}\right\}$, each symbol is carrying $M$ bits and $O=2^{M} \cdot \mathbf{H}_{k}$ is the 


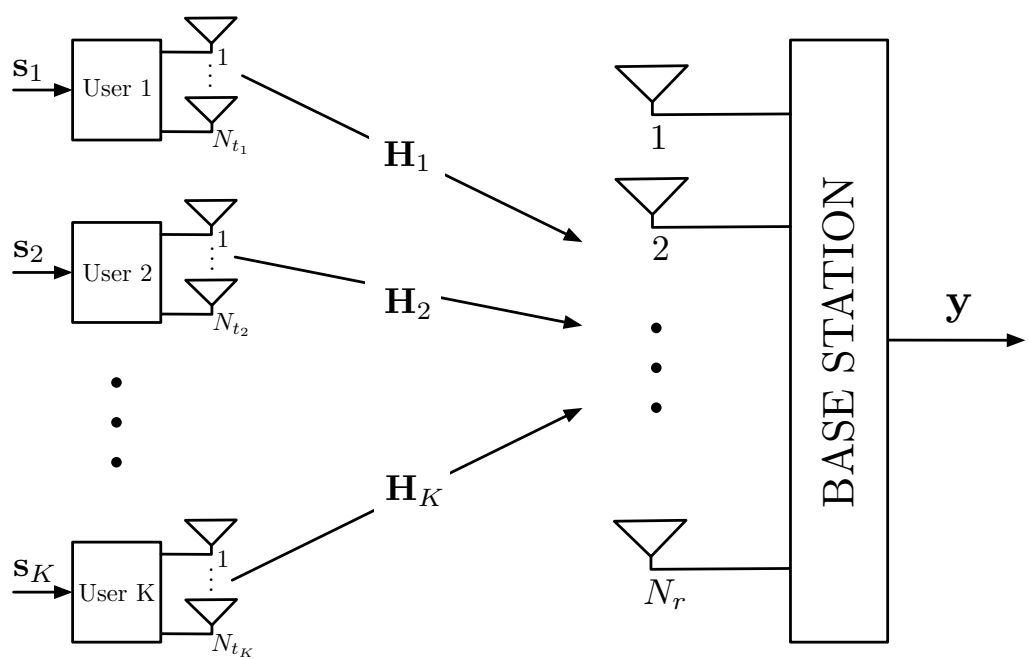

Figure 2.1: Multiuser MIMO system.

$N_{r} \times N_{t_{k}}$ channel matrix of the $k$-th user with elements $h_{i, j}^{(k)}$ corresponding to the complex channel gain from the $j$-th transmit antenna of user $k$ to the $i$-th receive antenna. The vector $\mathbf{n}$ is an $N_{r} \times 1$ zero mean complex circular symmetric Gaussian noise vector with covariance matrix $\mathbf{K}_{\mathbf{n}}=\mathbb{E}\left[\mathbf{n n}^{\mathcal{H}}\right]=\sigma_{n}^{2} \mathbf{I}$. The expression in (2-1) can be written more conveniently as

$$
\mathbf{y}=\mathbf{H s}+\mathbf{n} .
$$

where $\mathbf{H}=\left[\begin{array}{llll}\mathbf{H}_{1} & \mathbf{H}_{2} \ldots \mathbf{H}_{K}\end{array}\right]$ and $\mathbf{s}=\left[\begin{array}{lll}\mathbf{s}_{1}^{T} & \mathbf{s}_{2}^{T} \ldots \mathbf{s}_{K}^{T}\end{array}\right]^{T}$. The symbol vector $\mathbf{s}$ of all $K$ users has zero mean and a covariance matrix $\mathbf{K}_{\mathbf{s}}=\mathbb{E}\left[\mathbf{s s}^{\mathcal{H}}\right]=\sigma_{s}^{2} \mathbf{I}$, where $\sigma_{s}^{2}$ is the signal power of each transmit antenna. We assume that the channel matrix $\mathbf{H}$ was previously estimated at the BS.

\section{3}

\section{MIMO Channel Capacity}

Channel capacity is the upper bound on the rate of information that can be reliably transmitted over a communications channel. The channel capacity of MIMO systems can be increased by the factor $\min \left(N_{t}, N_{r}\right)$ when compared to a conventional single-antenna system, for the same transmit power and spectral bandwidth $[16,17]$. In this section, the MIMO channel capacities are derived for both deterministic and random channels. The MIMO channel capacity is defined as

$$
C=\max _{p_{\mathbf{s}}(\mathbf{s})} \mathbf{I}(\mathbf{s} ; \mathbf{y})
$$

where $p_{\mathbf{s}}(\mathbf{s})$ is the probability density function of the transmitted vector $\mathbf{s}$, and $\mathbf{I}(\mathbf{s} ; \mathbf{y})$ is the mutual information of the random vectors $\mathbf{s}$ and $\mathbf{y}$, given by 


$$
\mathbf{I}(\mathbf{s} ; \mathbf{y})=H(\mathbf{y})-H(\mathbf{y} \mid \mathbf{s})=H(\mathbf{y})-H(\mathbf{n})
$$

where $H(\mathbf{y})$ represents the differential entropy of $\mathbf{y}$ and $H(\mathbf{y} \mid \mathbf{s})$ is the conditional differential entropy of $\mathbf{y}$ when $\mathbf{s}$ is given. As the vectors $\mathbf{s}$ and $\mathbf{n}$ are statistically independent, $H(\mathbf{y} \mid \mathbf{s})=H(\mathbf{n})$. The differential entropy of the vector $\mathbf{y}$ is defined by

$$
H(\mathbf{y})=-\int_{\mathbf{R}^{N}} f(\mathbf{y}) p_{\mathbf{y}}(\mathbf{y}) d \mathbf{y}=-\mathbb{E}[f(\mathbf{y})],
$$

where $p_{\mathbf{y}}(\mathbf{y})$ is the probability density function of the vector $\mathbf{y}$ and $f(\mathbf{y})=$ $\log _{2}\left(p_{\mathbf{y}}(\mathbf{y})\right)$. The differential entropy $H(\mathbf{y})$ is maximized when $\mathbf{y}$ is a complex circularly-symmetric Gaussian random vector with mean $\mathbf{m}_{\mathbf{y}}=0$, which consequently requires $\mathbf{s}$ in (2-2) to be zero-mean circularly-symmetric complex Gaussian. Under this condition, the probability density function of $\mathbf{y}$ is given by

$$
p_{\mathbf{y}}(\mathbf{y})=\frac{1}{\pi^{N_{r}} \operatorname{det}\left(\mathbf{K}_{\mathbf{y}}\right)} e^{-\mathbf{y}^{\mathcal{H}} \mathbf{K}_{\mathbf{y}}^{-1} \mathbf{y}}
$$

where $\mathbf{K}_{\mathbf{y}}$ is the covariance matrix of $\mathbf{y}$ and $N_{r}$ is the number of elements in $\mathbf{y}$. Thus, taking the logarithm of (2-6), using $\mathbf{K}_{\mathbf{y}}=\mathbf{R}_{\mathbf{y}}$, where $\mathbf{R}_{\mathbf{y}}$ is the autocorrelation matrix of the vector $\mathbf{y}$, and $\mathbb{E}\left[\mathbf{y}^{\mathcal{H}} \mathbf{K}_{\mathbf{y}}^{-1} \mathbf{y}\right]=\mathbb{E}\left[\mathbf{y}^{\mathcal{H}} \mathbf{R}_{\mathbf{y}}^{-1} \mathbf{y}\right]=$ $\mathbb{T}\left\{\mathbb{E}\left[\mathbf{R}_{\mathbf{y}}^{-1} \mathbf{y} \mathbf{y}^{\mathcal{H}}\right]\right\}=\mathbb{T}\left\{\mathbf{R}_{\mathbf{y}}^{-1} \mathbf{R}_{\mathbf{y}}\right\}=\mathbb{T}\left[\mathbf{I}_{N_{r}}\right]=N_{r}$, where $\mathbb{T}[\mathbf{A}]$ denotes the trace of the matrix $\mathbf{A}$, we arrive at

$$
-\mathbb{E}[f(\mathbf{y})]=H(\mathbf{y})=\log _{2}\left(\pi^{N_{r}} e^{N_{r}} \operatorname{det}\left(\mathbf{R}_{\mathbf{y}}\right)\right)=\log _{2}\left(\operatorname{det}\left(\pi e \mathbf{R}_{\mathbf{y}}\right)\right) \mathrm{bps} / \mathrm{Hz},
$$

the differential entropy of the vector $\mathbf{n}$ is calculated similarly

$$
H(\mathbf{n})=\log _{2}\left(\operatorname{det}\left(\pi e \sigma_{n}^{2} \mathbf{I}_{N_{r}}\right)\right) \operatorname{bps} / \mathrm{Hz} .
$$

In order to obtain expressions for the channel capacity as a function of the energy $E_{\mathbf{s}}$ of the signal vector $\mathbf{s}$, a normalized version of (2-2) is used here

$$
\mathbf{y}=\sqrt{\frac{E_{\mathbf{s}}}{N_{t}}} \mathbf{H} \mathbf{s}+\mathbf{n},
$$

where $\frac{E_{\mathbf{s}}}{N_{t}}$ is the average energy per transmitted symbol. The autocorrelation matrix of $\mathbf{y}$ in (2-9) is given by

$$
\mathbf{R}_{\mathbf{y}}=\frac{E_{\mathbf{s}}}{N_{t}} \mathbf{H R}_{\mathbf{s}} \mathbf{H}^{\mathcal{H}}+N_{0} \mathbf{I}_{N_{r}},
$$

where the matrix $\mathbf{R}_{\mathbf{s}}$ with dimension $N_{t} \times N_{t}$ is the autocorrelation matrix of the information vector $\mathbf{s}$ normalized $\left(\mathbb{E}\left[\|\mathbf{s}\|^{2}\right]=\mathbb{T}\left[\mathbf{R}_{\mathbf{s}}\right]=N_{t}\right)$ and $N_{0}$ is the unilateral power spectral density of the noise at the receiver. Considering the expressions (2-3)-(2-10) the channel capacity of the MIMO channel is expressed as

$$
\mathbf{C}=\max _{\mathbb{T}\left[\mathbf{R}_{\mathbf{s}}\right]=N_{t}} \log _{2}\left[\operatorname{det}\left(\mathbf{I}_{N_{r}}+\frac{E_{\mathbf{s}}}{N_{t} N_{0}} \mathbf{H R}_{\mathbf{s}} \mathbf{H}^{\mathcal{H}}\right)\right] \mathrm{bps} / \mathrm{Hz}
$$




\subsection{1}

\section{Channel Capacity when Channel State Information is not Available at the Transmitter Side}

It is assumed here that the energy is distributed equally along the transmit antennas and the components of the vector $\mathbf{s}$ are statistically independent. Thus, the autocorrelation matrix of the vector information $\mathbf{s}$ is $\mathbf{R}_{\mathbf{s}}=\mathbf{I}_{N_{t}}$. In this case the expression for the channel capacity becomes

$$
\mathbf{C}=\log _{2}\left[\operatorname{det}\left(\mathbf{I}_{N_{r}}+\frac{E_{\mathbf{s}}}{N_{t} N_{0}} \mathbf{H H}^{\mathcal{H}}\right)\right] \mathrm{bps} / \mathrm{Hz}
$$

The matrix $\mathbf{H}$ has a singular value decomposition (SVD) given by $\mathbf{H}=\mathbf{U} \mathbf{\Sigma} \mathbf{V}^{\mathcal{H}}$, where $\mathbf{U}$, matrix with the left singular vectors, and $\mathbf{V}$, matrix with the right singular vectors, are square unitary matrices, $\mathbf{U} \mathbf{U}^{\mathcal{H}}=\mathbf{I}_{N_{r}}$, with dimensions $N_{r}$ and $N_{t}$, respectively, $\boldsymbol{\Sigma}$ is a rectangular matrix with dimension $N_{r} \times N_{t}$ and its diagonal elements are the singular values of the matrix $\mathbf{H}$ denoted by $\sigma_{1}, \sigma_{2}, \ldots, \sigma_{N_{\min }}$, where $N_{\min }=\min \left(N_{t}, N_{r}\right)$. Thus we have

$$
\mathbf{H H}^{\mathcal{H}}=\mathbf{U} \Sigma \Sigma^{\mathcal{H}} \mathbf{U}^{\mathcal{H}}=\mathbf{Q} \Lambda \mathbf{Q}^{\mathcal{H}}
$$

where $\mathbf{Q}=\mathbf{U}$ and $\Lambda$ is a diagonal matrix whose diagonal elements are the eigenvalues $\left\{\lambda_{i}=\sigma_{i}^{2}\right\}$ of the matrix $\mathbf{H H}^{\mathcal{H}}$. Thus, equation (2-12) can be expressed as

$$
\mathbf{C}=\log _{2}\left[\operatorname{det}\left(\mathbf{I}_{N_{r}}+\frac{E_{s}}{N_{t} N_{0}} \mathbf{Q} \Lambda \mathbf{Q}^{\mathcal{H}}\right)\right] \mathrm{bps} / \mathrm{Hz}
$$

However, we have

$$
\log _{2}\left[\operatorname{det}\left(\mathbf{Q}\left(\mathbf{I}_{N_{r}}+\frac{E_{s}}{N_{t} N_{0}} \Lambda\right) \mathbf{Q}^{\mathcal{H}}\right)\right]=\log _{2}\left[\operatorname{det}\left(\mathbf{I}_{N_{r}}+\frac{E_{s}}{N_{t} N_{0}} \Lambda\right) \operatorname{det}\left(\mathbf{Q Q}^{\mathcal{H}}\right)\right],
$$

and $\mathbf{Q}$ is a unitary matrix, then $\mathbf{Q Q}^{\mathcal{H}}=\mathbf{I}_{N_{r}}$ and $\operatorname{det}\left(\mathbf{I}_{N_{r}}\right)=1$, the channel capacity reduces to

$$
\mathbf{C}=\log _{2}\left[\operatorname{det}\left(\mathbf{I}_{N_{r}}+\frac{E_{\mathbf{s}}}{N_{t} N_{0}} \boldsymbol{\Lambda}\right)\right] \mathrm{bps} / \mathrm{Hz}
$$

Equation (2-16) can be rewritten alternatively as the sum of the capacities of $N_{\text {min }}$ SISO channels with power $E_{\mathrm{s}} / N_{\text {min }}$

$$
\mathbf{C}=\sum_{i=1}^{N_{\min }} \log _{2}\left(1+\frac{E_{\mathbf{s}}}{N_{t} N_{0}} \lambda_{i}\right) \mathrm{bps} / \mathrm{Hz} .
$$




\subsection{2}

\section{Channel Capacity when Channel State Information is Available at the Transmitter Side}

If the channel state information is available at the transmitter side a pre-processing, using the singular value decomposition (SVD), can be done in the transmitter as shown in Figure 2.2. The transmitted signal $\mathbf{s}$ is the

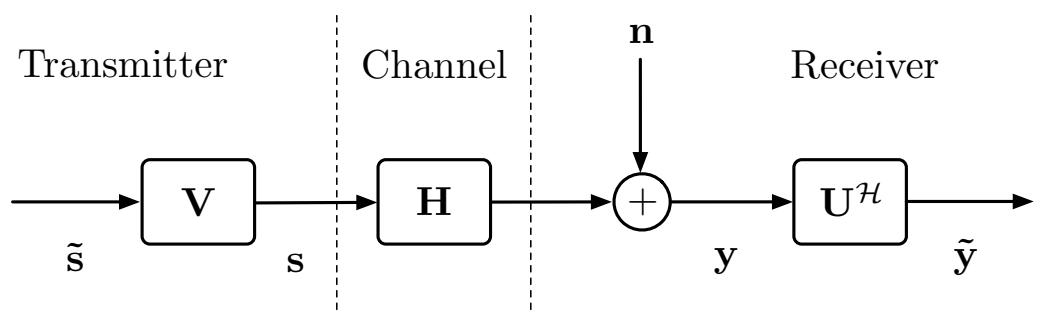

Figure 2.2: Decomposition of $\mathbf{H}$ when CSI is available at the transmitter side.

result of the pre-multiplication of the information vector $\tilde{\mathbf{s}}$ by the matrix $\mathbf{V}$, the received signal vector $\mathbf{y}$ is pre-multiplied by $\mathbf{U}^{\mathcal{H}}$, where $\mathbf{U}$ and $\mathbf{V}$ are the unitary matrices defined in the previous subsection. Thus, the received signal vector is

$$
\begin{aligned}
\tilde{\mathbf{y}} & =\sqrt{\frac{E_{\mathbf{s}}}{N_{t}}} \mathbf{U}^{\mathcal{H}} \mathbf{H V} \tilde{\mathbf{s}}+\mathbf{U}^{\mathcal{H}} \mathbf{n} \\
& =\sqrt{\frac{E_{\mathbf{s}}}{N_{t}}} \Sigma \tilde{\mathbf{s}}+\tilde{\mathbf{n}}
\end{aligned}
$$

where $\tilde{\mathbf{n}}$ is a complex circularly-symmetric Gaussian noise vector with zero mean, dimension $N_{r} \times 1$ and autocorrelation matrix $\mathbf{R}_{\mathbf{n}}=N_{0} \mathbf{I}_{N_{r}}$. Equation (218) can be expressed as $N_{r}$ SISO signals in parallel

$$
\tilde{y}_{i}=\sqrt{\frac{E_{\mathbf{s}}}{N_{t}}} \sqrt{\lambda_{i}} \tilde{s}_{i}+\tilde{n}_{i}, \quad i=1,2, \ldots, N_{r} .
$$

Therefore, the mutual entropy of the MIMO channel for this particular case can be expressed as the sum of $N_{r}$ SISO channels

$$
\mathbf{I}=\sum_{i=1}^{N_{r}} \log _{2}\left(1+\frac{E_{\mathbf{s}} \gamma_{i}}{N_{t} N_{0}} \lambda_{i}\right) \mathrm{bps} / \mathrm{Hz}
$$

where $\gamma_{i}=E\left[\left|\tilde{s}_{i}\right|^{2}\right]$ is the energy at the $i$-th SISO channel. Assuming that the eigenvalues $\lambda_{i}$ are ordered such as $\lambda_{i}>\lambda_{i-1}$, the MIMO channel capacity can be expressed as

$$
\mathbf{C}=\max _{\sum_{i}^{r} \gamma_{i}=N_{t}} \sum_{i=1}^{r} \log _{2}\left(1+\frac{E_{\mathbf{s}} \gamma_{i}}{N_{t} N_{0}} \lambda_{i}\right) \mathrm{bps} / \mathrm{Hz}
$$


where $r \leq \min \left(N_{r}, N_{t}\right)$ is the rank of $\mathbf{H H}^{\mathcal{H}}$.

\subsection{3}

\section{Deterministic SIMO Channel Capacity}

In SIMO channels, $N_{t}=1$, the channel matrix is a column matrix given by

$$
\mathbf{H}=\left(h_{1} h_{2} \ldots h_{N_{r}}\right)^{T} .
$$

Thus, $\mathbf{H}^{\mathcal{H}} \mathbf{H}$ has rank $1(r=1)$ with the only non-zero eigenvalue given by $\lambda_{1}=\mathbf{H}^{\mathcal{H}} \mathbf{H}=\sum_{i=1}^{N_{r}}\left|h_{i}\right|^{2}$.

\section{SIMO Channel Capacity When CSI Is Not Available at the Transmitter Side}

The channel capacity of equation (2-17) becomes

$$
\mathbf{C}=\log _{2}\left(1+\sum_{i=1}^{N_{r}}\left|h_{i}\right|^{2} \frac{E_{\mathbf{s}}}{N_{0}}\right) \operatorname{bps} / \mathrm{Hz}
$$

if the elements of the channel matrix are normalized as $\left|h_{1}\right|^{2}=\left|h_{2}\right|^{2}=\ldots$ $\left|h_{N_{r}}\right|^{2}=1$ the capacity in (2-23) when CSI is not available to the transmitted side is

$$
\mathbf{C}=\log _{2}\left(1+N_{r} \frac{E_{\mathbf{s}}}{N_{0}}\right) \text { bps } / \mathrm{Hz}
$$

\section{SIMO Channel Capacity when CSI is Available at the Transmitter Side}

As $\sum_{i}^{r} \gamma_{i}=\gamma_{1}=N_{t}=1$ and $\lambda_{1}=\sum_{i=1}^{N_{r}}\left|h_{i}\right|^{2}$, the channel capacity of equation (2-21) becomes

$$
\mathbf{C}=\log _{2}\left(1+\sum_{i=1}^{N_{r}}\left|h_{i}\right|^{2} \frac{E_{\mathbf{s}}}{N_{0}}\right) \text { bps/Hz. }
$$

Comparing (2-25) and (2-23), it is concluded that the knowledge of the channel state information does not influence the SIMO channel capacity.

\subsection{4}

\section{Deterministic MISO Channel Capacity}

In MISO channels, $N_{r}=1$ and $N_{t}$ transmit antennas, the channel is represented by a row matrix given by

$$
\mathbf{H}=\left(\begin{array}{llll}
h_{1} & h_{2} & \ldots & h_{N_{t}}
\end{array}\right),
$$

with $\mathbf{H H}^{\mathcal{H}}=\sum_{j=1}^{N_{t}}\left|h_{j}\right|^{2}$. 


\section{MISO Channel Capacity when CSI is not Available to the Transmitter Side}

From the channel capacity in equation (2-12) we have

$$
\mathbf{C}=\log _{2}\left(1+\sum_{j=1}^{N_{t}}\left|h_{j}\right|^{2} \frac{E_{\mathbf{s}}}{N_{t} N_{0}}\right) \operatorname{bps} / \mathrm{Hz}
$$

if the elements of the channel matrix are normalized as $\sum_{j=1}^{N_{t}}\left|h_{j}\right|^{2}=N_{t}$ the channel capacity in (2-27) becomes

$$
\mathbf{C}=\log _{2}\left(1+\frac{E_{\mathbf{s}}}{N_{0}}\right) \text { bps } / \mathrm{Hz}
$$

We note that channel capacity in (2-28) is the same as that the SISO channel capacity, that is, the capacity does not increase with the number of transmit antennas.

\section{MISO Channel Capacity when CSI is Available at the Transmitter Side}

Since matrix $\mathbf{H H}^{\mathcal{H}}$ has only one eigenvalue $(r=1)$ given by

$$
\lambda_{1}=\sum_{i=1}^{N_{t}}\left|h_{i}\right|^{2}
$$

and as $\sum_{i}^{r} \gamma_{i}=\gamma_{1}=N_{t}$, the channel capacity given by (2-21) becomes

$$
\mathbf{C}=\log _{2}\left(1+\frac{E_{\mathbf{s}}}{N_{0}} \sum_{i=1}^{N_{t}}\left|h_{i}\right|^{2}\right) \mathrm{bps} / \mathrm{Hz} .
$$

If the elements of the channel matrix are normalized as $\sum_{i=1}^{N_{t}}\left|h_{i}\right|^{2}=N_{t}$, the channel capacity in (2-30) becomes

$$
\mathbf{C}=\log _{2}\left(1+N_{t} \frac{E_{\mathbf{s}}}{N_{0}}\right) \text { bps } / \mathrm{Hz}
$$

Hence, for MISO channels there is a difference between the channel capacity when the CSI is available or not at the transmitter side.

\subsection{5}

\section{Channel Capacity of Random MIMO Channels}

Now consider the case in which the components of the channel matrix are modeled as complex random variables. Thus, if the channel is random the mutual entropy is also random. The ergodic channel capacity is defined as the expected value of the channel capacity conditioned on the channel matrix $\mathbf{H}$ :

$$
\mathbf{C}=\mathbb{E}\{\mathbf{C}(\mathbf{H})\}=\mathbb{E}\left\{\max _{\mathbb{T}\left[\mathbf{R}_{\mathbf{s}}\right]=N_{t}} \log _{2}\left[\operatorname{det}\left(\mathbf{I}_{N_{r}}+\frac{E_{\mathbf{s}}}{N_{t} N_{0}} \mathbf{H R}_{\mathbf{s}} \mathbf{H}^{\mathcal{H}}\right)\right]\right\} \mathrm{bps} / \mathrm{Hz} .
$$


for the case in which the CSI is not available at the received side, using (2-17), the MIMO channel capacity becomes

$$
\mathbf{C}=\mathbb{E}\left[\sum_{i=1}^{N_{\min }} \log _{2}\left(1+\frac{E_{\mathbf{s}}}{N_{t} N_{0}} \lambda_{i}\right)\right] \mathrm{bps} / \mathrm{Hz},
$$

where the eigenvalues $\lambda_{1}, \lambda_{2}, \ldots, \lambda_{\min }$ are random variables. When the CSI is available at the transmitter side the ergodic channel capacity is given by the expected value of the results in (2-21).

\section{4}

\section{Least Squares MIMO Channel Estimation}

In this section the least squares (LS) channel estimation algorithm for MIMO system proposed in [73] is reviewed. In the LS algorithm the cost function in the time instant $i$ must be defined based on a weighted average of error squares as:

$$
\mathcal{J}[i]=\sum_{k=1}^{i} \lambda^{i-k}\|\mathbf{y}[k]-\hat{\mathbf{H}}[i] \mathbf{s}[k]\|^{2},
$$

where $\mathbf{y}[k]$ and $\mathbf{s}[k]$ are the received and transmitted symbol vectors in the time instant $k$, respectively, $\lambda$ is the forgetting factor, $\hat{\mathbf{H}}[i]$ is the channel matrix estimate in the time instant $i$. The cost function is minimized by solving $\nabla_{\hat{\mathbf{H}}[i]} \mathcal{J}[i]=\mathbf{0}_{N_{r}, N_{t}}$, where $\mathbf{0}_{N_{r}, N_{t}}$ denotes the $N_{r} \times N_{t}$ zero matrix. The LS estimate of the channel matrix can be obtained by

$$
\hat{\mathbf{H}}[i]=\mathbf{D}[i] \mathbf{P}[i]
$$

where $\mathbf{D}[i]$ can be iteratively calculated by

$$
\mathbf{D}[i]=\lambda \mathbf{D}[i-1]+\mathbf{y}[i] \mathbf{s}[i]^{\mathcal{H}} .
$$

The matrix $\mathbf{P}[i]$ can be calculated iteratively by using the matrix inversion lemma as

$$
\mathbf{P}[i]=\lambda^{-1} \mathbf{P}[i-1]-\frac{\lambda^{-2} \mathbf{P}[i-1] \mathbf{s}[i] \mathbf{s}[i]^{\mathcal{H}} \mathbf{P}[i-1]}{1+\lambda^{-1} \mathbf{s}[i]^{\mathcal{H}} \mathbf{P}[i-1] \mathbf{s}[i]}
$$

Initially, we set the parameters $\mathbf{D}[0]=\mathbf{0}_{N_{r}, N_{t}}$ and $\mathbf{P}[0]=\varepsilon^{-1} \mathbf{I}$ where $\varepsilon$ is a small constant.

\section{5}

\section{Detection Techniques}

The main challenge in communication systems is to obtain an acceptable estimate of the transmitted information at the receiver side. In this section we examine the most important existing signal detection techniques for MU- 
MIMO systems, considering the received signal vector as in (2-2). To detect the user's data stream we assume that the channel state information (CSI) at the BS was previously estimated.

\subsection{1}

\section{Linear Detection}

In linear detectors, the received signal vector from all users, $\mathbf{y}$, is filtered by a linear filter to reduce the channel effects [33]. The linear detectors have a lower computational complexity when compared with the non-linear detectors, however, due to the impact of interference and noise, linear detectors offer a limited performance.

\section{Zero Forcing Detector}

Zero Forcing (ZF) detection assumes that the number of receive antennas, $N_{r}$, is greater or equal to the number of transmitted symbols of all users, $N_{t}$, i.e. $N_{r} \geq N_{t}$. The ZF receive filter that cancels the interference between antennas in the received signal is given by

$$
\mathbf{W}_{z f}=\left(\mathbf{H}^{\mathcal{H}} \mathbf{H}\right)^{-1} \mathbf{H}^{\mathcal{H}} .
$$

In other words, the ZF receive filter eliminates channel effects on the transmitted signal without concern about noise. The estimated symbol vector is given by

$$
\begin{aligned}
\hat{\mathbf{s}}_{z f} & =\mathbf{W}_{z f} \mathbf{y} \\
& =\left(\mathbf{H}^{\mathcal{H}} \mathbf{H}\right)^{-1} \mathbf{H}^{\mathcal{H}}(\mathbf{H} \mathbf{s}+\mathbf{n}) \\
& =\mathbf{s}+\tilde{\mathbf{n}}_{z f},
\end{aligned}
$$

where $\tilde{\mathbf{n}}_{z f}=\left(\mathbf{H}^{\mathcal{H}} \mathbf{H}\right)^{-1} \mathbf{H}^{\mathcal{H}} \mathbf{n}$. The ZF hard decision of $\mathbf{s}$ is carried out as follow

$$
\hat{\mathbf{s}}=\mathbb{C}\left(\hat{\mathbf{s}}_{z f}\right),
$$

where the function $\mathbb{C}(x)$ returns the point of the complex signal constellation closest to $x$. When $\mathbf{H}$ is nearly singular, the noise term, $\mathbf{n}_{z f}$, in (2-39) is enhanced and performance degrades.

\section{Minimum Mean Square Error Detector}

In order to maximize the signal-to-interference-plus-noise ratio, i.e. reduce the effects of the noise and the interference between antennas in the received signal, the minimum mean square error (MMSE) detector, that takes into account also the noise component, is obtained by minimizing the mean- 
square error (MSE) as

$$
\begin{aligned}
\mathbf{W}_{\text {mmse }} & =\arg \min _{\mathbf{W}} \mathbb{E}\left[\|\mathbf{s}-\mathbf{W} \mathbf{y}\|^{2}\right] \\
& =\mathbb{E}\left[\mathbf{s} \mathbf{y}^{\mathcal{H}}\right]\left(\mathbb{E}\left[\mathbf{y} \mathbf{y}^{\mathcal{H}}\right]\right)^{-1} \\
& =\mathbf{H}^{\mathcal{H}}\left(\mathbf{H} \mathbf{H}^{\mathcal{H}}+\sigma^{2} \mathbf{I}\right)^{-1} \\
& =\left(\mathbf{H}^{\mathcal{H}} \mathbf{H}+\sigma^{2} \mathbf{I}\right)^{-1} \mathbf{H}^{\mathcal{H}}
\end{aligned}
$$

where $\sigma^{2}=\sigma_{n}^{2} / \sigma_{s}^{2}$. The estimated symbol vector is given by

$$
\begin{aligned}
\hat{\mathbf{s}}_{m m s e} & =\mathbf{W}_{m m s e} \mathbf{y} \\
& =\left(\mathbf{H}^{\mathcal{H}} \mathbf{H}+\sigma^{2} \mathbf{I}\right)^{-1} \mathbf{H}^{\mathcal{H}}(\mathbf{H} \mathbf{s}+\mathbf{n}) .
\end{aligned}
$$

Finally the MMSE hard decision of $\mathbf{s}$ is obtained from $\hat{\mathbf{s}}_{m m s e}$ by

$$
\hat{\mathbf{s}}=\mathbb{C}\left(\hat{\mathbf{s}}_{m m s e}\right) .
$$

\subsection{2}

\section{Non Linear Detection}

In this section different nonlinear detection techniques are reviewed. First the optimal Maximum Likelihood (ML) detector is studied, then an approximation of the ML detector known as Sphere Decoder is presented. Then, a detection scheme that cancels the multi-antenna interference without changing the noise characteristics, called successive interference cancellation (SIC) is presented.

\section{Maximum Likelihood Detector}

For a given observation $\mathbf{y}$, the decision rule that minimizes the probability of error

$$
P(e)=P(\mathbf{s} \neq \hat{\mathbf{s}})
$$

is to choose the estimate, $\hat{\mathbf{s}}_{\mathrm{MAP}}$, that minimizes the conditional probability

$$
P(\mathbf{s}=\hat{\mathbf{s}} \mid \mathbf{y})=\frac{P(\mathbf{s}=\hat{\mathbf{s}}) p_{\mathbf{y} \mid \mathbf{s}}(\mathbf{y} \mid \mathbf{s}=\hat{\mathbf{s}})}{p_{\mathbf{y}}(\mathbf{y})}
$$

where $p_{\mathbf{y}}(\mathbf{y})$ is the probability density function of the observation $\mathbf{y}$ and $p_{\mathbf{y} \mid \mathbf{s}}(\mathbf{y} \mid \mathbf{s}=\hat{\mathbf{s}})$ is the probability density function of $\mathbf{y}$ when the transmitted signal is $\hat{\mathbf{s}}$. Since $p_{\mathbf{y}}(\mathbf{y})$ does not depend on $\hat{\mathbf{s}}$, the MAP estimate $\hat{\mathbf{s}}_{\mathrm{MAP}}$ can be 
obtained by

$$
\begin{aligned}
\hat{\mathbf{s}}_{\mathrm{MAP}} & =\arg \max _{\hat{\mathbf{s}} \in \mathcal{A}^{N_{t}}} P(\mathbf{s}=\hat{\mathbf{s}} \mid \mathbf{y}) \\
& =\arg \max _{\hat{\mathbf{s}} \in \mathcal{A}^{N_{t}}} P(\mathbf{s}=\hat{\mathbf{s}}) p_{\mathbf{y} \mid \mathbf{s}}(\mathbf{y} \mid \mathbf{s}=\hat{\mathbf{s}}),
\end{aligned}
$$

where $\mathcal{A}$ denotes the modulation constellation and $N_{t}$ is the number of transmit antennas. If the transmitted signals all have the same a priori probability, the MAP detector is equivalent to the ML detector

$$
\hat{\mathbf{s}}_{m l}=\arg \max _{\hat{\mathbf{s}} \in \mathcal{A}^{N}} p_{\mathbf{y} \mid \mathbf{s}}(\mathbf{y} \mid \mathbf{s}=\hat{\mathbf{s}}) .
$$

Considering expression (2-2), we have that

$$
p_{\mathbf{y} \mid \mathbf{s}}(\mathbf{y} \mid \mathbf{s}=\hat{\mathbf{s}})=p_{\mathbf{n}}(\mathbf{y}-\mathbf{H} \hat{\mathbf{s}})
$$

where the probability density function of the white Gaussian noise $\mathbf{n}$ is

$$
p_{\mathbf{n}}(\mathbf{n})=\frac{1}{\left(\pi \sigma_{n}^{2}\right)^{N_{r}}} e^{\frac{-1}{\sigma_{n}^{2}}\|\mathbf{n}\|^{2}} .
$$

Then results from (2-48) and (2-49) that $p_{\mathbf{y} \mid \mathbf{s}}$ is maximized with the minimization of $\|\mathbf{y}-\mathbf{H} \hat{\mathbf{s}}\|^{2}$, therefore the ML estimate of $\mathbf{s}$, i.e. $\hat{\mathbf{s}}_{m l}$ is given by

$$
\hat{\mathbf{s}}_{m l}=\arg \min _{\hat{\mathbf{s}} \in \mathcal{A}^{N_{t}}}\|\mathbf{y}-\mathbf{H} \hat{\mathbf{s}}\|^{2} .
$$

Thus, the ML detector selects the message $\hat{\mathbf{s}}$ with the smallest Euclidean distance between the received signal vector $\mathbf{y}$ and the hypothesis message $\mathbf{H} \hat{\mathbf{s}}$. The ML detector has a computational complexity that increases with the modulation order and exponentially with the number of transmitting antennas.

\section{Sphere Decoder}

The ML detector in (2-50) can be alternatively rewritten using the decomposition $\mathbf{H}=\mathbf{Q R}$, where $\mathbf{Q}$ is an orthogonal matrix, $\mathbf{Q Q}^{\mathcal{H}}=\mathbf{I}$, and $\mathbf{R}$ is an upper triangular matrix

$$
\hat{\mathbf{s}}_{m l}=\arg \min _{\hat{\mathbf{s}} \in \mathcal{A}^{N_{t}}}\|\hat{\mathbf{y}}-\mathbf{R} \hat{\mathbf{s}}\|^{2},
$$

where $\hat{\mathbf{y}}=\mathbf{Q}^{\mathcal{H}} \mathbf{y}$. The Sphere Decoder (SD), introduced originally in [29], consider that the real valued transformation [74] was previously applied in the observation vector $\mathbf{y}$. The real valued transformation converts the channel matrix, the transmitted signal vector and the noise vector to real values. Without loss of generality, we consider that the dimensions of the receiver signal vector after the real valued transformation have the same dimensions than (2-2) with all its elements are real values. The main idea of the SD [30-32] is to solve (2-51) considering all the points inside a hyper-sphere with the radius 
$d$ centered in $\hat{\mathbf{y}}$, i.e., all $\hat{s}$ that satisfy

$$
\|\hat{\mathbf{y}}-\mathbf{R} \hat{\mathbf{s}}\|^{2} \leq d^{2}
$$

SD considers only a small set of vectors within a hyper-sphere rather than all possible transmitted signal vectors. SD adjusts the hyper-sphere radius until there exists a single vector within a hyper-sphere:

- Increases the radius when there is no vector inside the hyper-sphere.

- Decreases the radius when there are multiple vectors inside the hypersphere.

Equation (2-52) can be rewritten as

$$
\sum_{i=1}^{N_{t}}\left(\sum_{j=1}^{N_{t}} \hat{y}_{i}-r_{i, j} \hat{s}_{j}\right)^{2} \leq d^{2}
$$

where $r_{i, j}$ is the $i$-th row and $j$-th column value of the upper triangular matrix R. Equation (2-53) can be expanded as follows

$$
\left(\hat{y}_{N_{t}}-r_{N_{t}, N_{t}} \hat{s}_{N_{t}}\right)^{2}+\left(\hat{y}_{N_{t}-1}-r_{N_{t}-1, N_{t}} \hat{s}_{N_{t}}-r_{N_{t^{-1}, N_{t}-1}} \hat{s}_{N_{t^{-1}}}\right)^{2}+\cdots \leq d^{2},
$$

the first term in equation (2-54) depends only on $\hat{s}_{N_{t}}$, the second term depends on $\hat{s}_{N_{t}}$ and $\hat{s}_{N_{t^{-1}}}$, and so on. We can see that a necessary condition for R $\hat{\mathbf{s}}$ to be inside the hyper-sphere is $\left(\hat{y}_{N_{t}}-r_{N_{t}, N_{t}} \hat{s}_{N_{t}}\right)^{2} \leq d^{2}$ which is equivalent to the following condition for $\hat{s}_{N_{t}}$ :

$$
\frac{\hat{y}_{N_{t}}-d}{r_{N_{t}, N_{t}}} \leq \hat{s}_{N_{t}} \leq \frac{\hat{y}_{N_{t}}+d}{r_{N_{t}, N_{t}}} .
$$

For simplicity, we define

$$
d_{N_{t}-1}^{2}=d^{2}-\left(\hat{y}_{N_{t}}-r_{N_{t}, N_{t}} \hat{s}_{N_{t}}\right)^{2}
$$

and

$$
\hat{y}_{N_{t^{-1}}}^{1}=\hat{y}_{N_{t^{-1}}}-r_{N_{t^{-1}, N_{t}}} \hat{s}_{N_{t}},
$$

thus, for $\left(\hat{y}_{N_{t}-1}^{1}-r_{N_{t^{-1}, N_{t}-1}} \hat{s}_{N_{t^{-1}}}\right)^{2} \leq d_{N_{t^{-1}}}^{2}$ the condition for $\hat{s}_{N_{t^{-1}}}$ turns

$$
\frac{\hat{y}_{N_{t}-1}^{1}-d_{N_{t}-1}}{r_{N_{t}-1, N_{t}-1}} \leq \hat{s}_{N_{t^{-1}}} \leq \frac{\hat{y}_{N_{t^{-1}}}^{1}+d_{N_{t^{-1}}}}{r_{N_{t^{-1}}, N_{t^{-1}}}} \text {. }
$$

Using the previous procedure the intervals for $\hat{s}_{N_{t}-2}, \hat{s}_{N_{t}-3}$ can be obtained, until $\hat{s}_{1}$ and thus all the points within the hyper-sphere can be determined. If a candidate does not exist in the intervals determined by the inequalities in (2-55) and (2-58), it will not be possible to keep searching for the next symbols. Instead, it is necessary to go back to the upper symbol and choose another candidate in the previous interval, then calculate the interval of the next symbol. When there are no values that satisfy all the inequalities it means 
that it is necessary to increase the initial radius of the hyper-sphere and restart the search. It turns out that there can be more than one possible solution of the transmitted signal vector inside the hyper-sphere, therefore the Euclidean distances between the candidates and $\hat{\mathbf{y}}$ must be calculated and the vector with the smallest distance is selected as the solution.

For a more simple visualization of the SD detector a tree diagram is shown in Figure 2.3. Looking for a possible solution from the symbol $s_{N_{t}}$ to the symbol $s_{1}$. It is supposed that the symbols between $s_{N_{t}}$ and $s_{k}$ have been found within the hyper-sphere, then for all $s_{k}$ all the possible $s_{k-1}$ are searched inside the hyper-sphere. For the example, in Figure 2.3, with $N_{t}=3$, at the top of the

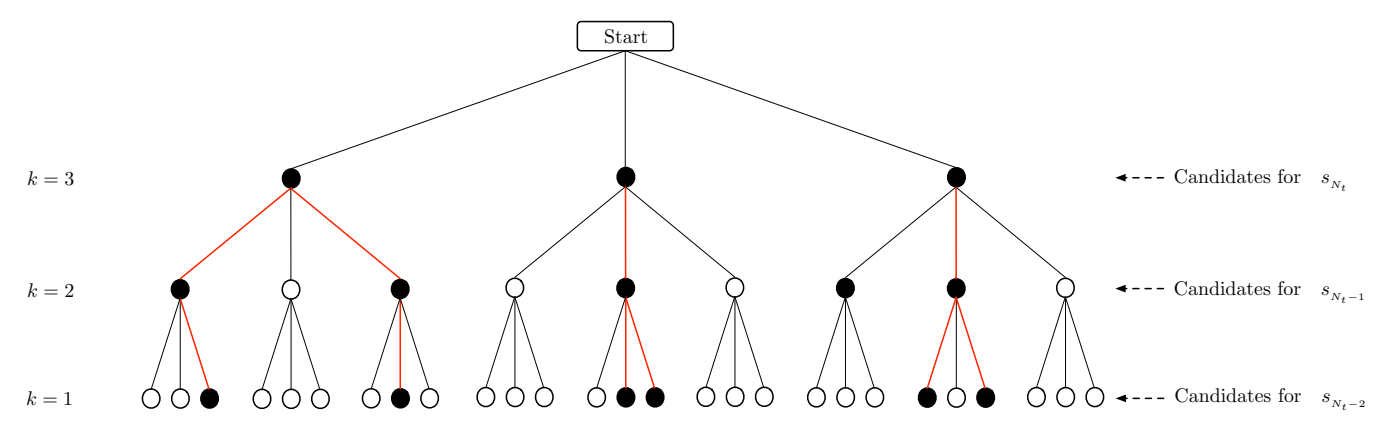

Figure 2.3: Tree diagram for Sphere Decoding

tree is necessary to find all possibles $s_{N_{t}}$, for this case there are three candidates for $s_{N_{t}}$. Then for all $s_{N_{t}}$ we find all possibles $s_{N_{t}-1}$ inside the hyper-sphere. Note that some nodes in the level $k=2$ can not lead to potential nodes on the lower level. Therefore in each node, from the top level to the bottom level, the tree will assign a value for the corresponding symbol. In Figure 2.3 there are six possible solutions for the transmitted signal vector in the hyper-sphere.

\section{Successive Interference Cancellation Detector}

The performance of MIMO systems with linear detectors can be improved without significant increase in the computational complexity by using the successive interference cancellation (SIC) technique. The SIC detector consists of a bank of linear detectors, each detects a selected component $s_{i}$ of $\mathbf{s}$. The component obtained by the first detector is used to reconstruct the corresponding signal vector which is then subtracted from the received signal to further reduce the interference in the input to the next linear receive filter. The successively canceled received data vector that follows a chosen ordering in the $i$-th stage is given by

$$
\mathbf{y}_{i}=\mathbf{y}-\sum_{j=1}^{i-1} \mathbf{h}_{j} \hat{s}_{j}
$$


where $\mathbf{h}_{j}$ denotes the $j$-th column of the channel matrix $\mathbf{H}$ and $\hat{s}_{j}$ is the estimated symbol obtained in the output of the $j$-th linear detector. Figure 2.4 shows the SIC scheme, where $\hat{s}_{i}$ is the estimated symbol obtained in the output of the $i$-th linear detector which may not correspond to the estimate of the symbol transmitted by the $i$-th antenna, the choice depends on the order that the symbols are to be detected. Following the diagram we have that

$$
\begin{aligned}
\tilde{\mathbf{y}}_{1} & =\mathbf{y}-\mathbf{h}_{1} \hat{s}_{1} \\
& =\mathbf{h}_{1}\left(s_{1}-\hat{s}_{1}\right)+\mathbf{h}_{2} s_{2}+\ldots+\mathbf{h}_{N_{t}} s_{N_{t}}+\mathbf{n} .
\end{aligned}
$$

Then, a new $\tilde{\mathbf{H}}_{1}$ is calculated as described by

$$
\tilde{\mathbf{H}}_{1}=\left[\begin{array}{llll}
\mathbf{h}_{1} & \mathbf{h}_{2} \ldots \mathbf{h}_{i-1} & \mathbf{h}_{i+1} \ldots \mathbf{h}_{N_{t}}
\end{array}\right]
$$

where $N_{t}=\sum_{k=1}^{K} N_{t_{k}}$. The second linear detector uses the matrix $\tilde{\mathbf{H}}_{1}$, and the process is repeated $N_{t}$ times until all components of the estimated vector $\hat{\mathbf{s}}$ are found. If $\hat{s}_{i}=s_{i}$ the interference is successfully eliminated, if $\hat{s}_{i} \neq s_{i}$, then error

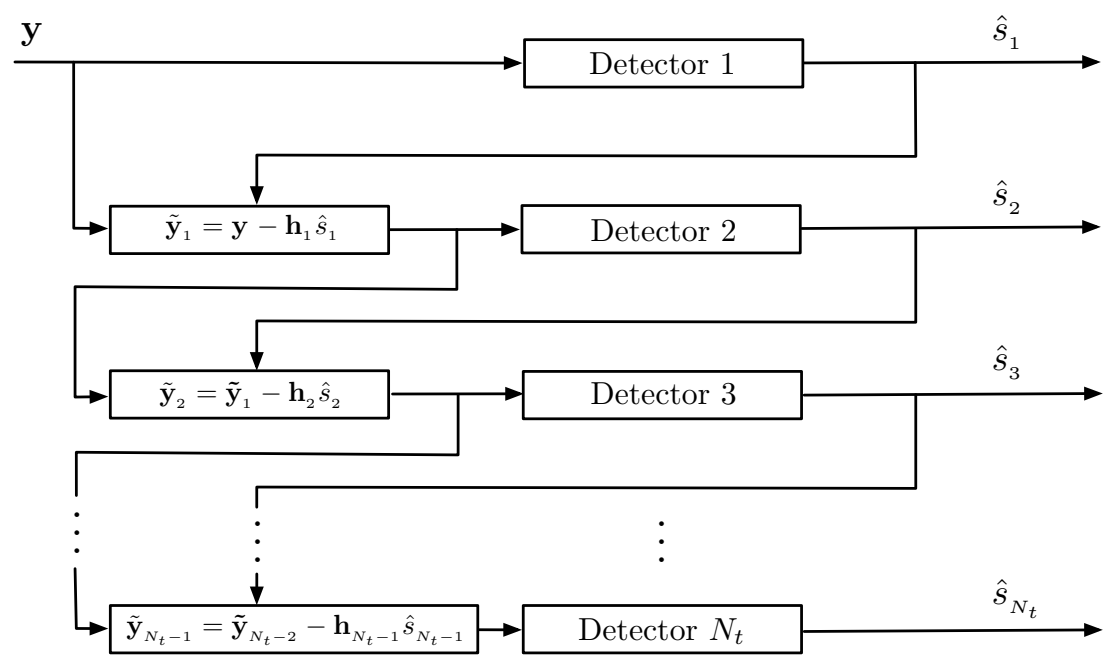

Figure 2.4: Successive Interference Cancellation Detector

propagation $[39,40]$ is incurred because the computation of the estimate of $s_{2}$ assumes that $\hat{s}_{1}=s_{1}$, i.e. that the interference was successfully canceled. The order of detection has a significant influence on the overall performance of SIC detection [34]. Different ordering methods are described here:

SINR Based Ordering Signals with higher signal-to-interference-plus-noiseratio (SINR) are detected first. If we consider linear MMSE detection, the estimation of the $i$-th symbol of the output of the first detector is obtained by 


$$
\hat{y}_{i}=\mathbf{w}_{i} \mathbf{h}_{1} s_{1}+\mathbf{w}_{i} \mathbf{h}_{i} s_{i}+\ldots+\mathbf{w}_{i} \mathbf{h}_{N_{t}} s_{N_{t}}+\mathbf{w}_{i} \mathbf{n},
$$

where $\mathbf{w}_{i}$ is the $i$-th row of the MMSE detection matrix in (2-41), thus the SINR in (2-62) is given by

$$
\mathrm{SINR}_{i}=\frac{\mathbb{E}\left[\left|\mathbf{w}_{i} \mathbf{h}_{i} s_{i}\right|^{2}\right]}{\mathbb{E}\left[\sum_{\substack{l=1 \\ l \neq i}}^{N_{t}}\left|\mathbf{w}_{i} \mathbf{h}_{l} s_{l}+\mathbf{w}_{i} \mathbf{n}\right|^{2}\right]}, i=1,2, \ldots, N_{t} .
$$

where $\mathbb{E}[$.$] is the expected value operator. As \mathbf{n}$ and $s_{l}$ are statistically independent, (2-63) becomes

$$
\operatorname{SINR}_{i}=\frac{E_{s_{i}}\left|\mathbf{w}_{i} \mathbf{h}_{i}\right|^{2}}{\sum_{\substack{l=1 \\ l \neq i}}^{N_{t}} E_{s_{l}}\left|\mathbf{w}_{i} \mathbf{h}_{l}\right|^{2}+\sigma_{\mathbf{n}}^{2}\left|\mathbf{w}_{i}\right|^{2}}, i=1,2, \ldots, N_{t} .
$$

where $E_{s_{l}}=\mathbb{E}\left[\left|s_{l}\right|^{2}\right]$ is the energy of the transmitted symbols. $N_{t}$ SINR values are calculated and the detected symbol that corresponds to the signal with the highest SINR is selected as $\hat{s}_{1}$ in Figure 2.4. Similar procedure is done to obtain $\hat{s}_{2}$ and so on.

SNR Based Ordering If we consider linear ZF detection, the interference components in (2-63) is suppressed. The estimation of the $i$-th symbol at the output of the first detector is then

$$
\hat{y}_{i}=s_{i}+\mathbf{w}_{i} \mathbf{n},
$$

where $\mathbf{w}_{i}$ is the $i$-th row of the ZF detection matrix in (2-38), thus the SINR in $(2-65)$ is reduced to

$$
S N R_{i}=\frac{\mathbb{E}\left[\left|s_{i}\right|^{2}\right]}{\mathbb{E}\left[\left|\mathbf{w}_{i} \mathbf{n}\right|^{2}\right]}=\frac{E_{s_{i}}}{\sigma_{\mathbf{n}}^{2}\left\|\mathbf{w}_{i}\right\|^{2}}, \quad i=1,2, \ldots, N_{t} .
$$

The same procedure described in the previous method is used to determine the detection ordering.

Column Norm-Based Ordering To reduce the computational complexity of the SINR and SNR based ordering schemes, a third method was proposed. The norm of the column vectors in the channel matrix is used. Consider the following representation of the received signal vector

$$
\mathbf{y}=\mathbf{H} \mathbf{s}+\mathbf{n}=\mathbf{h}_{1} s_{1}+\mathbf{h}_{2} s_{2}+\ldots+\mathbf{h}_{N_{t}} s_{N_{t}}+\mathbf{n},
$$

the signal power of the $i$-th received signal is proportional to the norm of the $i$-th column of the channel matrix. Therefore, the signals can be detected in decreasing order of the norms $\left\|\mathbf{h}_{i}\right\|$. 


\section{6}

\section{MIMO Detection by List Generation}

The linear detection schemes presented in the previous section often have a low performance due to the multiple access interference (MAI). On the other hand the non linear detectors such as SIC detector suffer from error propagation and performance degradation. In this section, we study two sub-optimal detection scheme for MU-MIMO, Multi-Branch Successive Interference Cancellation (MB-SIC) and Multiple Feedback Successive Interference Cancellation (MF-SIC). In these detectors, a list of tentative decisions of the transmitted signal is generated, then the best solution is selected using a selected criterion. In the MB-SIC detector a small set of reliable candidates for the transmitted signal vector are generated. In MF-SIC detector the selection procedure is constrained to one selected symbol in each spatial layer.

\subsection{1}

\section{Multi-Branch Successive Interference Cancellation Detection}

This subsection describes the MB-SIC detector $[36,37,75]$ for MU-MIMO system. In the multi-branch scheme, depicted in Figure 2.5, different orderings are explored for SIC, each ordering is referred to as a branch, so that a detector with $L$ branches produces a set of $L$ estimated vectors. Each branch uses a

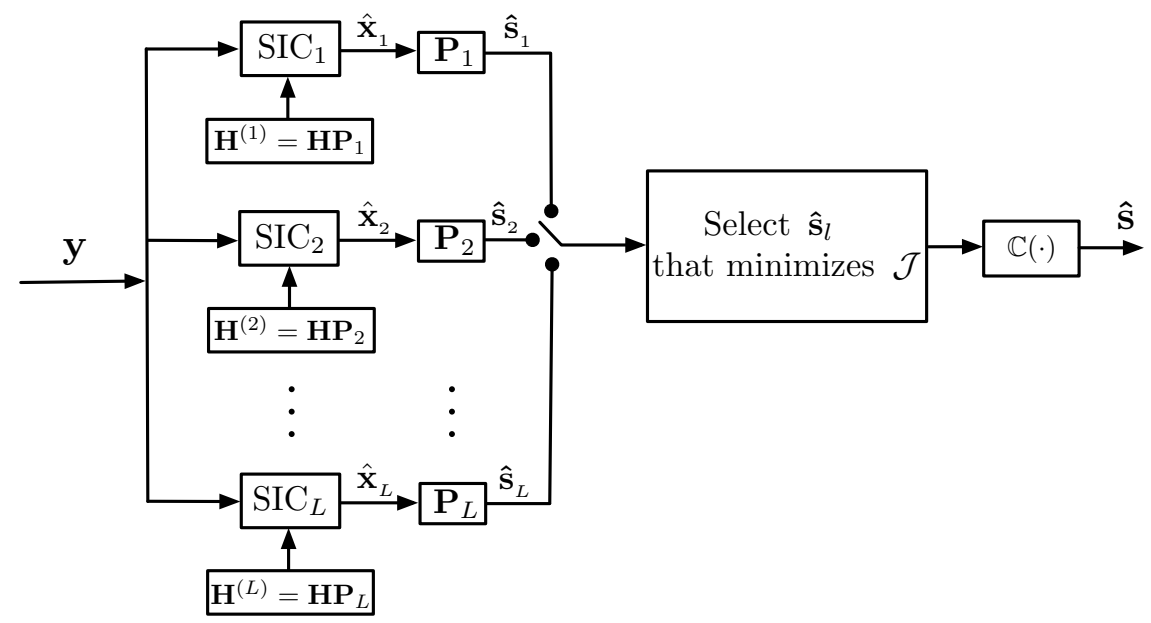

Figure 2.5: Block diagram of the MB-SIC detector.

column permutation matrix $\mathbf{P}$. The estimate of the signal vector of branch $l$, $\hat{\mathbf{x}}_{l}$, is obtained using a SIC receiver based on a new channel matrix

$$
\mathbf{H}^{(l)}=\mathbf{H P}_{l} \text {. }
$$

The order of the estimated symbols is rearranged to the original order by 


$$
\hat{\mathbf{s}}_{l}=\mathbf{P}_{l} \hat{\mathbf{x}}_{l}, \quad l=1, \ldots, L .
$$

A higher detection diversity can be obtained by selecting the most likely candidate symbol vector based on the ML selection rule.

\section{Selection Rule}

To select the branch with the best performance the Maximum Likelihood criterion is used. The cost function for the ML criterion is written as

$$
\mathcal{J}_{M L}=\left\|\mathbf{y}-\mathbf{H s}_{l}\right\|^{2} \quad l=1, \ldots, L .
$$

\section{Pre-Stored Patterns}

For the first branch the column permutation matrix $\mathbf{P}_{1}$ is chosen as the identity matrix $\mathbf{I}_{N t}$ to keep the optimal ordering. The remaining branches used the so called Pre-Stored Patterns (PSP) proposed in [75]. The PSP can be described mathematically by

$$
\mathbf{P}_{l}=\left[\begin{array}{cc}
\mathbf{I}_{s} & \mathbf{0}_{s, N_{t}-s} \\
\mathbf{0}_{N_{t}-s, s} & \phi\left[\mathbf{I}_{s}\right]
\end{array}\right], 2 \leqslant l \leqslant N_{t},
$$

where $\mathbf{0}_{m, n}$ denotes $m \times n$-dimensional matrix full of zeros, the operator $\phi[\cdot]$ rotates the elements of the argument matrix column-wise such that an identity matrix becomes a matrix with ones in the reverse diagonal. The PSP algorithm shifts the ordering of the cancellation according to shifts given by

$$
s=\left\lfloor(l-2) N_{t} / L\right\rfloor, 2 \leqslant l \leqslant N_{t},
$$

where $L$ is the number of parallel branches and $[$.$\rfloor rounds the argument to the$ lowest integer according to the $l$-th branch.

\subsection{2}

\section{Multiple Feedback Successive Interference Cancellation Detection}

The MF-SIC detector [38], depicted in Figure 2.6, uses a number of selected constellation points as the candidates when a previous decision is determined unreliable. The reliability of the previous detected symbol is determined by the Shadowing Area Constraints (SAC) algorithm, which saves the computational complexity by avoiding redundant processing with reliable decisions. The soft estimation of the $k$-th symbol is obtained by

$$
u_{k}=\mathbf{w}_{m m s e}^{k} \tilde{\mathbf{y}}_{k}
$$

where $\mathbf{w}_{m m s e}^{k}$ is the $N_{r} \times 1$ MMSE filter vector given by 


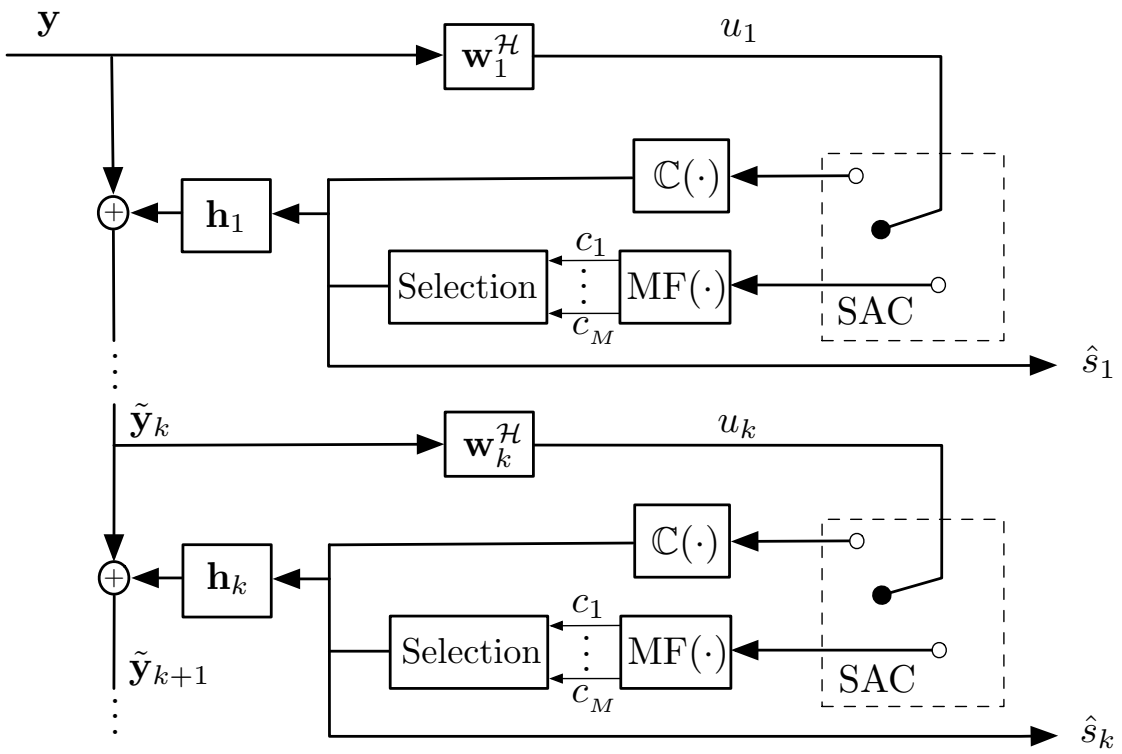

Figure 2.6: Block diagram of the MF-SIC detector.

$$
\mathbf{w}_{m m s e}^{k}=\left(\tilde{\mathbf{H}}_{k}^{\mathcal{H}} \tilde{\mathbf{H}}_{k}+\sigma_{n}^{2} \mathbf{I}\right)^{-1} \mathbf{h}_{k}^{\mathcal{H}}
$$

where $\tilde{\mathbf{H}}_{k}$ denotes the matrix obtained by taking the columns $k, k+1, \ldots, N_{t}$ of the channel matrix $\mathbf{H}$ and $\tilde{\mathbf{y}}_{k}$ is the received signal vector after the cancellation of $k-1$ previously detected symbols. Then the estimated symbol $u_{k}$ is checked by the SAC, which decides whether this decision is reliable according to the metric

$$
d_{k}=\min _{a_{f} \in \mathcal{A}}\left\{\left|u_{k}-a_{f}\right|\right\}
$$

where $a_{f}$ denotes the constellation point which is the nearest to the soft estimation symbol $u_{k}$.

For a predefined threshold $d_{t h}$, if $d_{k}>d_{t h}$ we say that $u_{k}$ is dropped into the shadowed area of the constellation map and this decision is determined unreliable, as shown in Figure 2.7. If $d_{k} \leqslant d_{t h}$ we say that $u_{k}$ is reliable.

\section{Decision Reliable}

If the soft estimation $u_{k}$ is considered reliable, the estimated symbol for each data stream $\hat{s}_{k}$ is obtained in the same way as in the conventional SIC detector by $\hat{s}_{k}=\mathbb{C}\left(u_{k}\right)$, where $\mathbb{C}($.$) is signal quantization. The hard decision symbol \hat{s}_{k}$ is considered as a reliable decision for the $k$-th symbol.

\section{Decision Unreliable}

If $u_{k}$ is unreliable, a candidate vector is generated

$$
\mathcal{L}=\left[c_{1}, c_{2}, \ldots, c_{m}, \ldots, c_{M}\right] \subseteq \mathcal{A},
$$




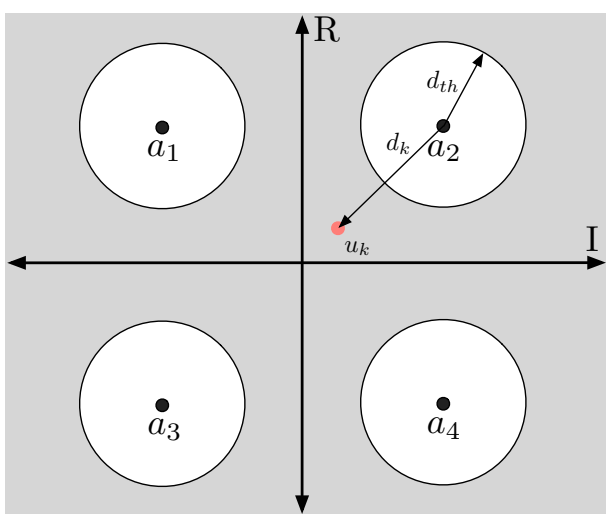

Figure 2.7: The shaded area is the unreliable region for QPSK constellation

where $\mathcal{L}$ contains the $M$ nearest to $u_{k}$ constellation points. The size of $\mathcal{L}$ can be determined by the signal-to-noise ratio (SNR). A higher SNR corresponds to a smaller $M$ which introduces a trade-off between the complexity and the performance. The unreliable decision $\mathbb{C}\left(u_{k}\right)$ is replaced by

$$
\hat{s}_{k}=c_{m_{\text {opt }}},
$$

where $c_{m_{o p t}}$ is the optimal candidate selected from the list $\mathcal{L}$. The selection algorithm of the optimal feedback candidate $c_{m_{o p t}}$ is described as follows: A set of selection vectors $\mathbf{b}^{1}, \ldots, \mathbf{b}^{m}, \ldots, \mathbf{b}^{M}$ is defined, the number of these selection vectors $M$ equals the number of constellation candidates we used for each unreliable decision. For the $k$-th layer, a $N_{t} \times 1$ vector $\mathbf{b}^{m}$ consists of the following elements,

- Previously detected symbols $\hat{s}_{1}, \ldots, \hat{s}_{k-1}$.

$-c_{m}$ a candidate symbol taken from the constellation for substituting the unreliable decision $\mathbb{C}\left(u_{k}\right)$ in the $k$-th layer.

- The detection of the following layers $k+1, \ldots, q, \ldots, N_{t}$ th is performed by the nulling and symbol cancellation which is equivalent to a traditional SIC algorithm.

Therefore, the vector is formed as follows

$$
\mathbf{b}^{m}=\left[\hat{s}_{1}, \ldots, \hat{s}_{k-1}, c_{m}, b_{k+1}^{m}, \ldots, b_{q}^{m}, \ldots, b_{N_{t}}^{m}\right]^{T},
$$

where $b_{q}^{m}$ is a potential decision that corresponds to the use of $c_{m}$ in the $k$-th layer,

$$
b_{q}^{m}=\mathbb{C}\left(\mathbf{w}_{q}^{\mathcal{H}} \hat{\mathbf{y}}_{q}^{m}\right),
$$

where $q$ indexes a certain layer from the $(k+1)$-th to the $N_{t}$-th. Finally 


$$
\hat{\mathbf{y}}_{q}^{m}=\tilde{\mathbf{y}}_{k}-\mathbf{h}_{k} c_{m}-\sum_{p=k+1}^{q-1} \mathbf{h}_{p} b_{p}^{m} .
$$

For each user, the same MMSE filter vector $\mathbf{w}_{k}$ is used for all candidates, which allows the proposed algorithm to have computational simplicity for the SIC detection. The candidate is selected according to

$$
m_{\text {opt }}=\arg \min _{1 \leqslant m \leqslant M}\left\|\mathbf{y}-\mathbf{H b}^{m}\right\|^{2} .
$$

The $c_{m_{\text {opt }}}$ is chosen to be the optimal feedback symbol for the next layer as well as more reliable decision for the current symbol.

\section{7 \\ Numerical Results}

The bit error rate (BER) performance of the different MIMO detectors, which include ZF, MMSE, SIC, MB-SIC, MF-SIC and ML are numerically compared in this section through computer simulations. We consider the uplink of a MU-MIMO system with $K$ active users. For all $K$ transmitters, the SNR per transmitted information bit is defined as

$$
\mathrm{SNR}=10 \log _{10} \frac{N_{t} \sigma_{s}^{2}}{M \sigma_{n}^{2}},
$$

where $\sigma_{s}^{2}$ is the common variance of the received symbols, $\sigma_{n}^{2}$ is the noise variance and $M$ is the number of information bits per transmitted symbol. In this chapter's simulations we consider independent and identically distributed random fading channel models whose coefficients are complex Gaussian random variables with zero mean and unit variance. The simulation curves correspond to an average of 3,000 simulation runs, with $500 N_{t}$ symbols transmitted per run.

Figure 2.8 shows the MIMO channel capacity, when CSI is not available at the transmitter side, versus SNR for different number of antennas obtained from (2-33). In Figure 2.8 we can see that the MIMO channel capacity improves when the number of transmit and receive antennas increases. In Figure 2.9 we compare the performance of the SIC detector for different ordering methods, SINR based ordering, SNR based ordering and the column norm-based ordering. It is observed that the performance with SINR based ordering have the best performance. In Figs $2.10-2.12$ we compare the performance of the existing detectors using QPSK and 16-QAM modulation. It is observed that the linear detectors, ZF and MMSE, provides the worst performance. We also note that SIC detector, despite having a better performance that the linear detectors still suffer from the error propagation. We can see that the sub- 


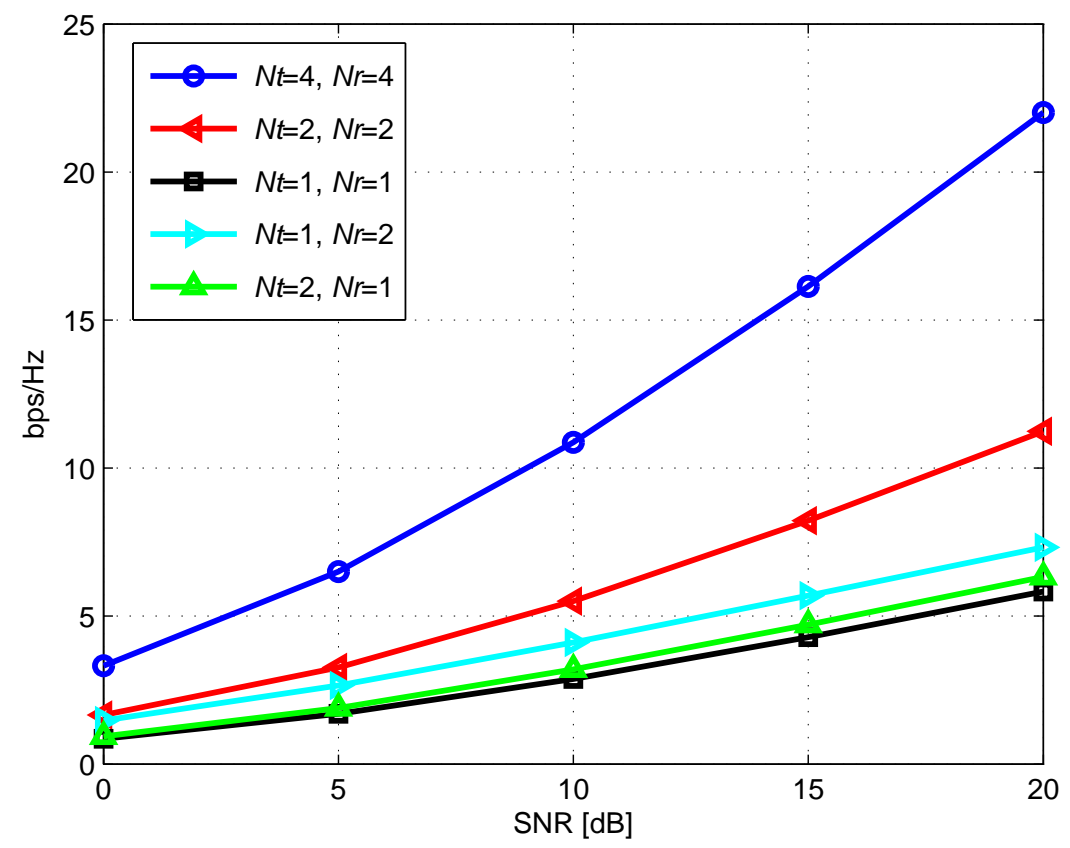

Figure 2.8: MIMO channel capacity when CSI is not available at the transmitter side.

optimal detectors by list generation, MB-SIC and MF-SIC, provide a trade-off between the performance and complexity, however, their performance is not comparable with the optimal performance, especially at high SNR values. It is necessary to improve the performance of these sub-optimal detectors. The optimal performance is obtained by the ML detector, with a full diversity gain, but the exhaustive search of the optimal solution leads to a high computational complexity which makes its implementation impractical. 


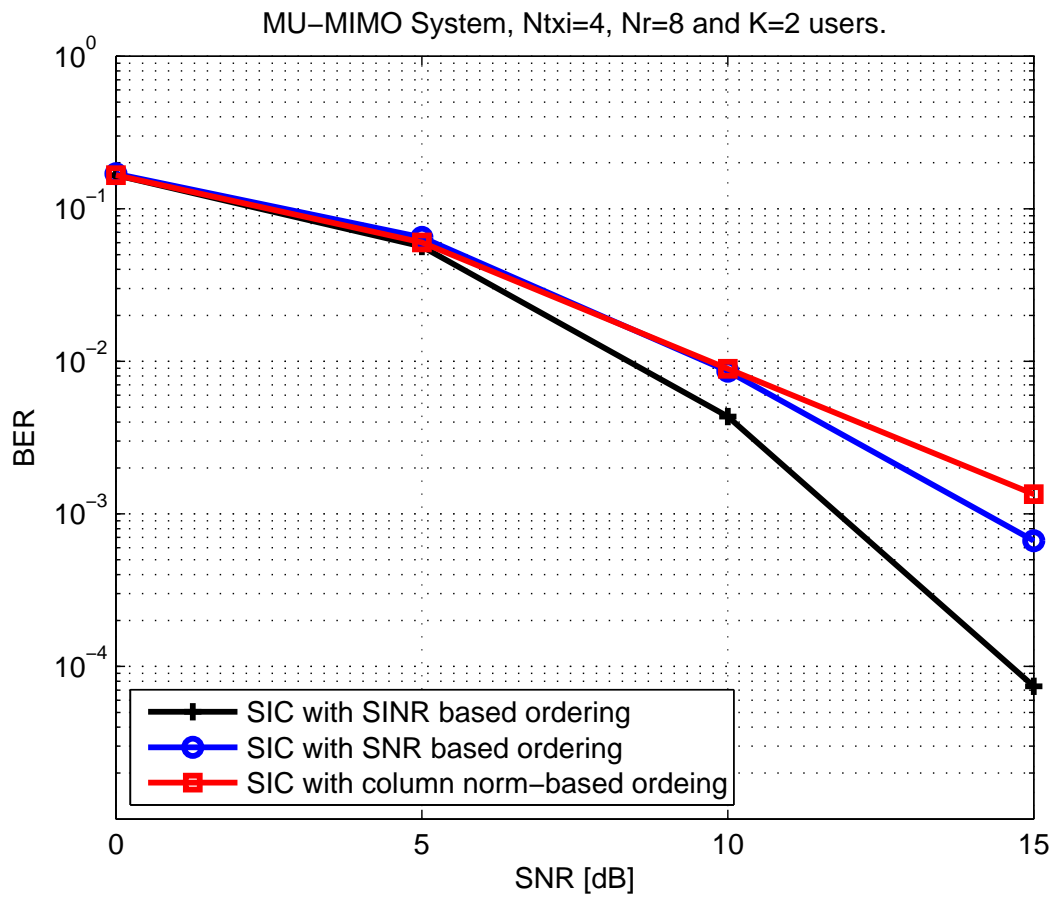

Figure 2.9: BER vs SNR of SIC detectors with different detection ordering, QPSK modulation, $K=2$ users and $N_{t_{i}}=4$ transmit antennas per user.

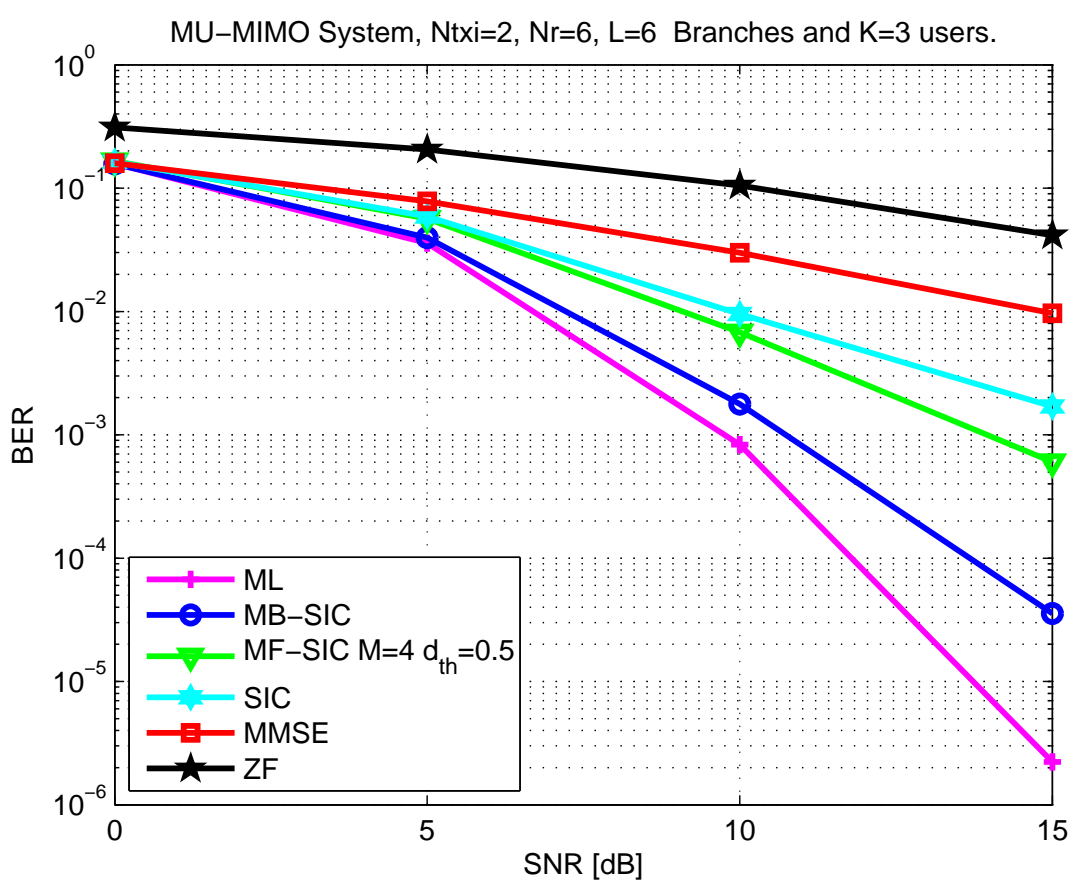

Figure 2.10: BER vs SNR of existing MU-MIMO detectors with QPSK modulation, $K=3$ users and $N_{t_{i}}=2$ transmit antennas per user. All SIC detectors use column-norm-based ordering. 


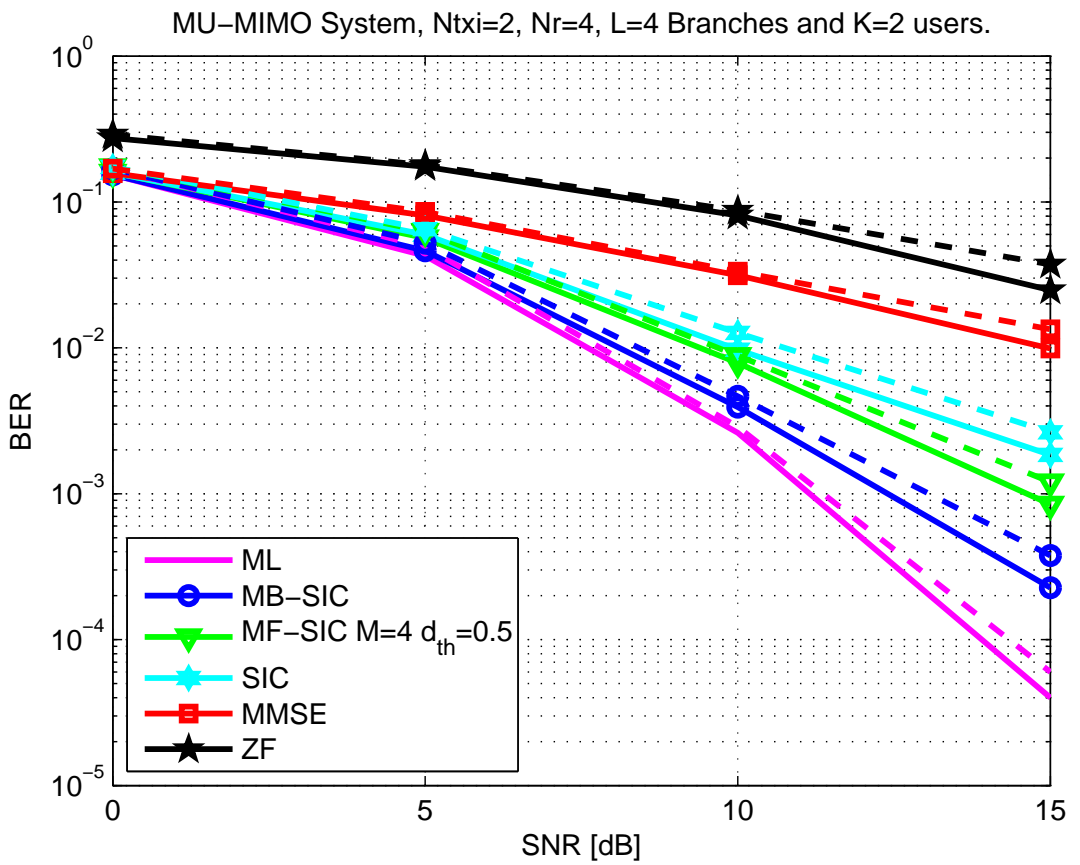

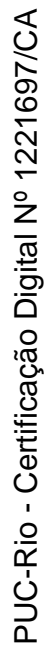

Figure 2.11: BER vs SNR of existing MU-MIMO detectors with QPSK modulation, $K=2$ users and $N_{t_{i}}=2$ transmit antennas per user. All SIC detectors use column-norm-based ordering. We also compared the performance with LS channel estimation (- -) and perfect channel estimation (-); $550 N_{t}$ symbols are transmitted, with 50 symbols used for training in the LS scheme.

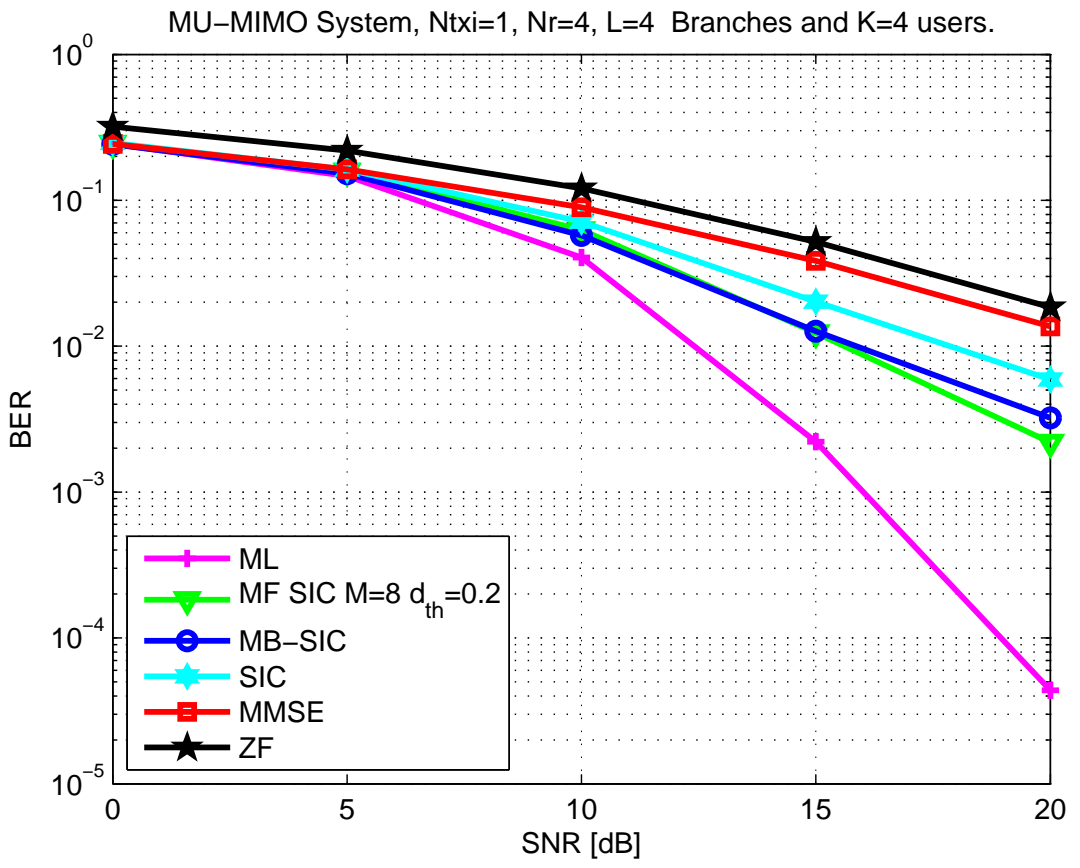

Figure 2.12: BER vs SNR of existing MU-MIMO detectors with 16-QAM modulation, $K=4$ users and $N_{t_{i}}=1$ transmit antennas per user. All SIC detectors use column-norm-based ordering. 


\section{Lattice Reduction Detection for MIMO Systems}

\section{1}

\section{Overview}

In the future generation of wireless communications systems, there will be a growing demand to reduce the weight and the power consumption, this requires reducing $\mathrm{MS}$ device sizes and BS equipment and efficient signal processing algorithms. However, the smaller spacing of antennas increases the correlation between antennas resulting in inferior detection performance [76]. In this Chapter a detection technique known as Lattice Reduction (LR) detection is presented. LR introduces a pre-processing in the estimated channel matrix at the receive side to reduce the effects of channel correlation $[41,42]$. The LR transformation finds a new basis for the channel matrix, which is nearly orthogonal allowing a more effective detection.

In this chapter an efficient implementation for the LR-SIC detector is proposed and evaluated. In this Chapter we also propose two new detection schemes for MU-MIMO systems in the uplink scenario. The first scheme proposed here combines LR, SIC and list detection schemes, that were reviewed in the previous chapter, to devise an MB-LR-SIC detector which obtains a good performance. The second proposed scheme, called VLD, tests a variable number of candidates for the transmitted symbol vector. An algorithm to decide if the current candidate has good quality or if it is necessary to further explore different orderings to improve the estimation quality is employed. The proposed VLD schemes have a near-optimal performance, while keeping under control the computational cost. We study the performance of the proposed and existing algorithms using a realistic MU-MIMO scenario with propagation effects and correlation between antennas.

This Chapter is structured as follows. Section 3.2 examines the LR concept. In Section 3.3 different detection schemes in the Lattice domain are described. The proposed MB-LR-SIC and VLD schemes are detailed in Section 3.4 and Section 3.5, respectively. Section 3.6 is dedicated to the presentation and discussion of the numerical results obtained via computer simulations. 


\section{2}

\section{Lattice Reduction Concept}

A lattice $\boldsymbol{\Lambda}$ may viewed as a regular tiling of a space. A lattice can be generated from an integer linear combination of a basis, i.e.,

$$
\Lambda=\left\{\mathbf{c} \mid \mathbf{c}=\sum_{m=1}^{M} \mathbf{b}_{m} z_{m}, z_{m} \in \mathbb{Z}\right\}
$$

where $\mathbb{Z}$ denotes the set of integer numbers and $\mathbf{b}_{m}, m=1, \ldots, M$ are realvalued and linearly independent vectors that form the basis

$$
\mathbf{B}=\left[\mathbf{b}_{1}, \mathbf{b}_{2}, \ldots, \mathbf{b}_{M}\right]
$$

An important property is that the same lattice $\boldsymbol{\Lambda}$ can be generated by different bases. If we ignore the noise vector in (2-2) we can see that it is possible to describe a MIMO system as a lattice under some considerations; The basis vectors of $\mathbf{H}$ should be real vectors and the elements of $\mathbf{s}$ need to be integers. Then, the system model in (2-2) can be described as a lattice spanned by the columns of $\mathbf{H}$.

In [52], the authors proposed a lattice where $\mathbf{H}$ and $\mathbf{s}$ have complex values. But the real and imaginary components of $\mathbf{s}$ still need to belong to a group of consecutive integers denoted by $\overline{\mathbb{Q}}=\mathbb{Z}+j \mathbb{Z}$. When the lattice basis $\mathbf{H}$ is orthogonal, the decision region of linear detectors is the same as the ML. Clearly, the orthogonality of $\mathbf{H}$ has an impact on the performance of MIMO detectors. To quantify the orthogonality of a matrix, a metric called orthogonal deficiency $(o d)$ is introduced in [77] and defined as

$$
\operatorname{od}(\mathbf{H})=1-\frac{\operatorname{det}\left(\mathbf{H}^{\mathcal{H}} \mathbf{H}\right)}{\prod_{n=1}^{N_{t}}\left\|\mathbf{h}_{n}\right\|^{2}},
$$

where $\mathbf{h}_{n}$ is the $n$-th column of $\mathbf{H}$ and $0 \leqslant \operatorname{od}(\mathbf{H}) \leqslant 1$. When $\operatorname{od}(\mathbf{H})$ is small, $\mathbf{H}$ is closer to an orthogonal matrix. As the same lattice can be generated by different bases, i.e., different channel matrices, it is possible to find a new basis $\tilde{\mathbf{H}}$ in the same lattice, which is nearly orthogonal or, at least, more orthogonal than $\mathbf{H}$. The bases $\mathbf{H}$ and $\tilde{\mathbf{H}}$ span the same lattice when the column vector of a basis $\tilde{\mathbf{H}}$ is an integer linear combination of the column vectors of the basis H. Then, we have

$$
\tilde{\mathbf{H}}=\mathbf{H T},
$$

where $\mathbf{T}$ is a uni-modular matrix $(\operatorname{det}|\mathbf{T}|=1)$. The received signal vector in $(2-2)$ can be rewritten as

$$
\mathbf{y}=\mathbf{H} \mathbf{s}+\mathbf{n}=\mathbf{H T T}^{-\mathbf{1}} \mathbf{s}+\mathbf{n}=\tilde{\mathbf{H}} \mathbf{z}+\mathbf{n},
$$


where $\mathbf{z}=\mathbf{T}^{\mathbf{- 1}} \mathbf{s}$. The estimation vector $\tilde{\mathbf{z}}$ of $\mathbf{z}$ can be computed using MIMO detectors based on the transformed matrix $\tilde{\mathbf{H}}$. To improve the performance of MIMO detection in (3-5) a nearly orthogonal basis $\tilde{\mathbf{H}}$ is desired. The matrix $\tilde{\mathbf{H}}$, generated from $\mathbf{H}$, is regarded as the lattice-reduced matrix. There are many lattice reduction algorithms to find the best basis vectors, the most important of these algorithms are presented next.

\subsection{1}

\section{Gaussian Lattice Reduction for a Two-Basis System}

In this subsection, a LR reduction for a simple case with a $2 \times 2$ basis is presented [43]. The objective is to find an LR matrix $\tilde{\mathbf{H}}=\left[\tilde{\mathbf{h}}_{1} \tilde{\mathbf{h}}_{2}\right]$ of the channel matrix $\mathbf{H}=\left[\begin{array}{ll}\mathbf{h}_{1} & \mathbf{h}_{2}\end{array}\right]$, under the assumption that

$$
\left\|\mathbf{h}_{1}\right\| \leqslant\left\|\mathbf{h}_{2}\right\|
$$

through the correlation reduction of $\mathbf{H}$. The condition in (3-6) can be guaranteed with column swapping. The columns of $\tilde{\mathbf{H}}$ are obtained by

$$
\begin{aligned}
\tilde{\mathbf{h}}_{1} & =\mathbf{h}_{1} \\
\tilde{\mathbf{h}}_{2} & =\mathbf{h}_{2}-\eta \mathbf{h}_{1},
\end{aligned}
$$

where $\eta$ is chosen such that it minimizes the inner product $\left\langle\tilde{\mathbf{h}}_{1}, \tilde{\mathbf{h}}_{2}\right\rangle$ which represents the correlation between $\tilde{\mathbf{h}}_{1}$ and $\tilde{\mathbf{h}}_{2}$

$$
\eta=\arg \min _{\eta \in \mathbb{Z}+j \mathbb{Z}}\left|\left\langle\tilde{\mathbf{h}}_{1}, \tilde{\mathbf{h}}_{2}\right\rangle\right|=\arg \min _{\eta \in \mathbb{Z}+j \mathbb{Z}}\left|\left\langle\mathbf{h}_{1}, \mathbf{h}_{2}\right\rangle-\eta\left\|\mathbf{h}_{1}\right\|^{2}\right|=\left\lfloor\frac{\left\langle\mathbf{h}_{1}, \mathbf{h}_{2}\right\rangle}{\left\|\mathbf{h}_{1}\right\|^{2}}\right\rceil,
$$

where $[\cdot]$ is the rounding operator, with the real and imaginary components being rounded separately. The vectors $\tilde{\mathbf{h}}_{1}$ and $\tilde{\mathbf{h}}_{2}$ may not be orthogonal, the next step is to find their correlation

$$
\begin{aligned}
\left\langle\tilde{\mathbf{h}}_{1}, \tilde{\mathbf{h}}_{2}\right\rangle & =\left\langle\mathbf{h}_{1},\left(\mathbf{h}_{2}-\left\lfloor\frac{\left\langle\mathbf{h}_{1}, \mathbf{h}_{2}\right\rangle}{\left\|\mathbf{h}_{1}\right\|^{2}}\right\rceil \mathbf{h}_{1}\right)\right\rangle \\
& =\left(\frac{\left\langle\mathbf{h}_{1}, \mathbf{h}_{2}\right\rangle}{\left\|\mathbf{h}_{1}\right\|^{2}}-\left\lfloor\frac{\left\langle\mathbf{h}_{1}, \mathbf{h}_{2}\right\rangle}{\left\|\mathbf{h}_{1}\right\|^{2}}\right\rceil\right)\left\|\mathbf{h}_{1}\right\|^{2} \\
& =\rho\left\|\mathbf{h}_{1}\right\|^{2},
\end{aligned}
$$

where $\rho$ represents the rounding error. Since the real and imaginary rounding errors will be no more than $\frac{1}{2}$, then we have from (3-10) that

$$
\left|\left\langle\tilde{\mathbf{h}}_{1}, \tilde{\mathbf{h}}_{2}\right\rangle\right| \leq \frac{1}{2}\left\|\tilde{\mathbf{h}}_{1}\right\|^{2} .
$$


Then, we check if the new vector $\tilde{\mathbf{h}}_{1}$ is shorter than $\tilde{\mathbf{h}}_{2}$, i.e., the condition in (36 ) is verified, if not so, swap them and repeat the procedure. The correlation between two vectors and the length of the vectors are checked in (3-11) and (3$6)$, respectively. If both conditions are fulfilled, we have the lattice-reduced matrix $\tilde{\mathbf{H}}$.

\subsection{2}

\section{Complex Lenstra - Lenstra - Lovasz Algorithm}

The Lenstra, Lenstra and Lov $\tilde{A}$ sz (LLL) lattice reduction algorithm has been the most popular LR algorithm in MIMO detection because it guarantees a bounded orthogonality defect [44,52]. LLL is a generalization of the Gaussian reduction for arbitrary bases dimensions, achieving an orthogonal basis via iterative size reduction operations, i.e., the lengths of the basis vectors are reduced, coupled with an appropriate column swapping if they are not in ascending order $[45,46]$. The complex-valued LLL (CLLL) proposed in [52] takes the $\mathrm{QR}$ decomposition of $\mathbf{H}$ and iteratively reduces the correlation between the basis vectors to produces a nearly-orthogonal basis $\tilde{\mathbf{H}}=\tilde{\mathbf{Q}} \tilde{\mathbf{R}}$ that satisfies:

$$
\begin{gathered}
\left|\operatorname{Re}\left(\tilde{R}_{l, k}\right)\right|,\left|\operatorname{Im}\left(\tilde{R}_{l, k}\right)\right| \leqslant \frac{1}{2}\left|\tilde{R}_{l, l}\right| \forall 1 \leq l \leq k \leq N_{t} \\
\delta\left|\tilde{R}_{k-1, k-1}\right|^{2} \leq\left|\tilde{R}_{k-1, k}\right|^{2}+\left|\tilde{R}_{k, k}\right|^{2} \quad \forall 2 \leq k \leq N_{t},
\end{gathered}
$$

where $\tilde{R}_{l, k}$ denotes the $(k, l)$-th entry of $\tilde{\mathbf{R}}$ and $\operatorname{Re}(x)$ and $\operatorname{Im}(x)$ represent the real and imaginary part of $x$, respectively. The factor $\delta \in\left[\frac{1}{4}, 1\right]$ controls the tradeoff between the processing time and the quality of the reduced basis. The common choice that achieves a good balance between speed and quality is $\delta=\frac{3}{4}$. The conditions in (3-12) and (3-13) are known as the size reduction and the LovA $\tilde{A}_{j}$ sz basis swapping condition, respectively. The CLLL reduction is summarized in Algorithm 1. The basis reduction operations to reduce the size of the $k$-th column of $\tilde{\mathbf{R}}$ against its previous $1: k-1$ columns are performed in lines 4-8. The rounding operator in line 5 refers to rounding to the nearest integer. After the size reduction, the Lov $\tilde{A}_{j}$ sz condition is checked for the $k$-th and $(k-1)$-th columns of $\tilde{\mathbf{R}}$, if it passes the two columns are swapped and Givens rotations are applied to maintain the upper-triangular nature of $\tilde{\mathbf{R}}$, otherwise the algorithm proceeds to the next column pair according to lines 9-14.

The orthogonality deficiency of $\tilde{\mathbf{H}}$, obtained by applying CLLL algorithm in $\mathbf{H}$ with rank $N_{t}$ for a given parameter $\delta$, satisfies the next condition

$$
\sqrt{1-\operatorname{od}(\tilde{\mathbf{H}})} \geqslant 2^{\frac{N_{t}}{2}}\left(\frac{2}{2 \delta-1}\right)^{-\frac{N_{t}\left(N_{t}+1\right)}{4}}:=c_{\delta}
$$




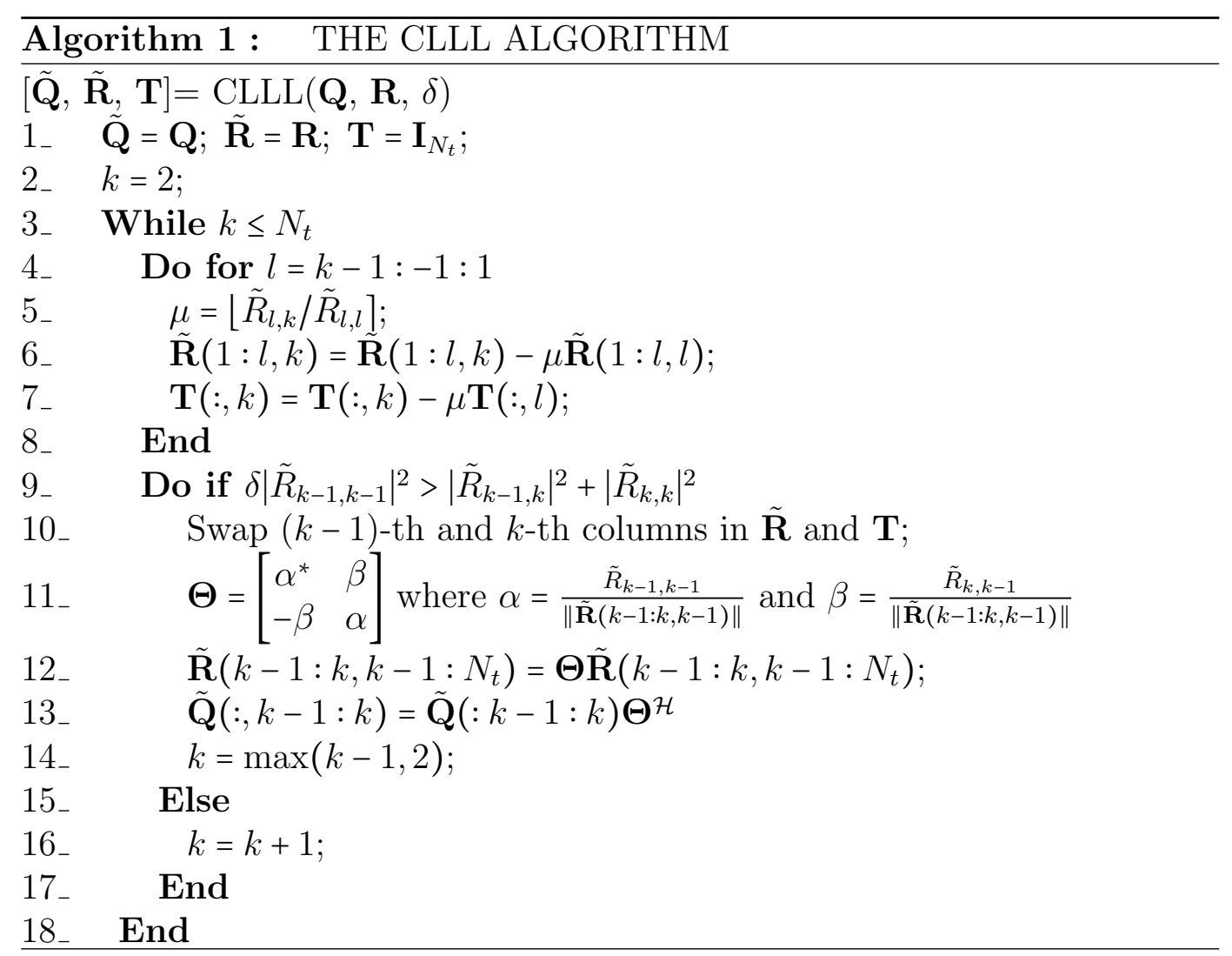

For any integer $N_{t} \geq 1, c_{\delta}$ is always less than 1 . Therefore, the orthogonality deficiency is bounded by

$$
\operatorname{od}(\tilde{\mathbf{H}})<1-c_{\delta}^{2} .
$$

From (3-14) and (3-15) we can see that although not guaranteeing to reduce the orthogonal deficiency for every realization of $\mathbf{H}$, the CLLL algorithm yields a new basis $\tilde{\mathbf{H}}$ whose orthogonal deficiency has an upper bound which is strictly less than one [54]. In previous works, the CLLL algorithm has shown an excellent trade-off between the orthogonal deficiency and the computational complexity.

\section{3}

\section{Lattice Reduction Detection}

In this section, some MIMO detection techniques in the lattice domain are studied, starting with the linear detectors such as LR Zero Forcing (LRZF) and LR Minimum Mean Square Error (LR-MMSE). We also review an important detection scheme, namely, the complex Lattice Reduction Successive Interference Cancellation (LR-SIC). An efficient implementation for LR-SIC is also proposed and evaluated in this section. 


\subsection{1}

\section{Lattice Reduction Linear Detection}

Linear detectors such as Zero Forcing (ZF) or minimum mean square error (MMSE) can be developed based on the lattice-reduced channel matrix $\tilde{\mathbf{H}}[53,54]$. The linear detectors in the lattice domain are given by

$$
\tilde{\mathbf{W}}^{\chi}= \begin{cases}\left(\tilde{\mathbf{H}}^{\mathcal{H}} \tilde{\mathbf{H}}\right)^{-1} \tilde{\mathbf{H}}^{\mathcal{H}}, & \chi=\mathrm{LR}-\mathrm{ZF} \\ \left(\tilde{\mathbf{H}}^{\mathcal{H}} \tilde{\mathbf{H}}+\sigma^{2} \mathbf{T}^{\mathcal{H}} \mathbf{T}\right)^{-1} \tilde{\mathbf{H}}^{\mathcal{H}}, & \chi=\text { LR-MMSE }\end{cases}
$$

the LR linear estimation of vector $\mathbf{z}$ is obtained by

$$
\tilde{\mathbf{z}}=\tilde{\mathbf{W}}^{\chi} \mathbf{y}
$$

Considering the MMSE case, an excellent alternative is to perform the LR transformation taking into account the noise amplification in the equalization procedure by applying the CLLL algorithm to the extended channel matrix:

$$
\overline{\mathbf{H}}=\left[\begin{array}{c}
\mathbf{H} \\
\sigma \mathbf{I}_{N_{t}}
\end{array}\right],
$$

rather than to $\mathbf{H}$. This solution improves the performance gain and reduces the computational complexity [53]. Along this thesis, we consider that the unimodular matrix $\mathbf{T}$ in (3-4) is calculated performing the LR transformation on the extended matrix $\overline{\mathbf{H}}$.

In practice, the components of the transmitted signal vector $\mathbf{s}$ are taken from a complex modulation, for that, the shifting and scaling operations should be used in the detected vector $\tilde{\mathbf{z}}$ [78]. The hard decision of $\mathbf{z}$ is found by

$$
\hat{\mathbf{z}}=\frac{1}{\alpha}\left(\left\lfloor\alpha \tilde{\mathbf{z}}+\beta \mathbf{T}^{-1} \mathbf{1}\right\rceil-\beta \mathbf{T}^{-1} \mathbf{1}\right),
$$

where $[\cdot]$ denotes the rounding operator, $\alpha$ and $\beta$ denote the scaling and shifting coefficients, respectively, and $\mathbf{1}$ is a vector of ones. Note that the shifting and scaling operations of $\hat{\mathbf{z}}$ would be unnecessary in the hypothetical case that $\mathbf{s} \in \overline{\mathbb{Q}}$. Finally, the hard decision of $\mathbf{s}$ is obtained by

$$
\hat{\mathbf{s}}=\mathbb{C}(\mathbf{T} \hat{\mathbf{z}}) .
$$

Satisfactory results have been obtained with LR linear detectors due to the reduced noise enhancement.

\subsection{2}

\section{Lattice Reduction Successive Interference Cancellation}

Even with a nearly orthogonal matrix $\tilde{\mathbf{H}}$, mutual interference between the components of the transformed signal $\mathbf{z}$ in (3-5) is still present. For this reason 
LR-SIC techniques result in additional improvements. The LR-SIC receiver consists of a bank of linear detectors based on the matrix $\tilde{\mathbf{H}}$, where each detects a selected component $z_{i}$ of $\mathbf{z}$. The component obtained by the first detector is used to reconstruct the corresponding signal vector, which is then subtracted from the received signal to further reduce the interference in the input to the next linear receive filter. The successively canceled data vector in the $i$-th stage is given by

$$
\mathbf{y}_{i}=\mathbf{y}-\sum_{j=1}^{i-1} \tilde{\mathbf{h}}_{j} \hat{z}_{j} .
$$

The estimate $\hat{z}_{i}$ of $z_{i}$ is obtained after shifting and scaling operations in the output, $\tilde{z}_{i}$, of the $i$-th linear detector:

$$
\hat{z}_{i}=\frac{1}{\alpha}\left\lfloor\alpha \tilde{z}_{i}+\beta \mathbf{t}_{i}^{-1} \mathbf{1}\right\rceil-\beta \mathbf{t}_{i}^{-1} \mathbf{1}, \quad i=1, \ldots, N_{t},
$$

where $\mathbf{t}_{i}^{-1}$ is the $i$-th row of $\mathbf{T}^{-1}$. The index of $z$ depends on the selected cancellation ordering, i.e., the order in which the interference vectors are subtracted. Then a new LR channel matrix is calculated removing the column vectors from the original $\tilde{\mathbf{H}}$ that corresponds to the symbols detected in the previous $i$ layers. The $i+1$ linear detector uses this new channel matrix. The process is repeated $N_{t}$ times until all components of the estimated vector $\hat{\mathbf{z}}$ are found. At the end of the detection procedure, the estimated transmit signal vector in the constellation domain can then be obtained by (3-20).

Since, due to the LR transformation, the components of $\mathbf{z}$ are correlated, the specific value of the detected symbols affects the variance of the symbols to be detected. The covariance of the LR symbols need to be updated at each stage. In [55], it is shown that the SIC with MMSE detection that considers the update of the symbols' covariance at each detection layer can be interpreted as applying the SIC algorithm above to an extended received signal given by

$$
\mathbf{y}_{e}=\left[\begin{array}{l}
\mathbf{y} \\
\mathbf{0}_{N_{t}}
\end{array}\right],
$$

in conjunction with the extended channel matrix

$$
\tilde{\mathbf{H}}_{e}=\left[\begin{array}{r}
\tilde{\mathbf{H}} \\
\sigma_{n} \mathbf{C}
\end{array}\right],
$$

where $\mathbf{0}_{N_{t}}$ denoted an all-zero vector of dimension $N_{t} \times 1$ and $\mathbf{C}=\sqrt{\mathbf{R}^{-1}}$, where $\mathbf{R}$ is the covariance matrix of vector $\mathbf{z}$, i.e., $\mathbf{R}=\mathbb{E}\left[\mathbf{z z}^{\mathcal{H}}\right]=\sigma_{s}^{2} \mathbf{T}^{-1} \mathbf{T}^{-\mathcal{H}}$. The linear filter at the first LR-SIC layer is given by the Moore-Penrose pseudo-inverse of $\tilde{\mathbf{H}}_{e}$ defined as $\tilde{\mathbf{H}}_{e}^{\dagger}=\left(\tilde{\mathbf{H}}_{e}^{\mathcal{H}} \tilde{\mathbf{H}}_{e}\right)^{-1} \tilde{\mathbf{H}}_{e}^{\mathcal{H}}$.

In this thesis, we employ an equivalent version of the LR-SIC detector described in (3-21)-(3-22) applied to the extended version in (3-23)-(3-24) that 
avoids the computational effort of calculating the matrix $\mathbf{C}=\sqrt{\mathbf{R}^{-1}}$. In our version, we replace the matrix $\mathbf{C}$ in $(3-24)$ with the matrix $\mathbf{T} / \sigma_{s}$. Then, we assume for simplicity of notation that the LR symbols are estimated according to the increasing order of their indexes, the estimated symbol in the $n$-th LRSIC layer is given by

$$
\tilde{z}_{n}=\left[\begin{array}{c}
\tilde{\mathbf{H}}_{[n]} \\
\sigma \mathbf{T}_{[n]}
\end{array}\right]_{n}^{\dagger}\left[\begin{array}{c}
\mathbf{y}-\tilde{\mathbf{H}}_{[n r]} \hat{\mathbf{z}}_{[n r]} \\
-\sigma \mathbf{T}_{[n r]} \hat{\mathbf{z}}_{[n r]}
\end{array}\right], n=1, \ldots, N_{t}
$$

where $\sigma=\sigma_{n} / \sigma_{s}$, [A $]_{n}^{\dagger}$ represents the $n$-th row of the Moore-Penrose pseudoinverse of $\mathbf{A}, \hat{\mathbf{z}}_{[n r]}$ is a vector formed by the already detected symbols at the $n$-th SIC stage and $\mathbf{A}_{[n]}$ and $\mathbf{A}_{[n r]}$ denote, respectively, matrices with the first $(n-1)$ and with the last $\left(N_{t}-n+1\right)$ columns of the matrix $\mathbf{A}$ removed. The equivalence between the two versions is demonstrated in Appendix A.

\section{4}

\section{Multi-Branch Lattice Reduction Successive Interference Cancellation De- tection}

This section describes the proposed Multi-Branch Lattice Reduction Successive Interference Cancellation (MB-LR-SIC) detection scheme that combines the concepts previously presented. The main idea is to employ an LR transformation on the channel matrix, then generate multiple branches, where each branch has a different ordering pattern and produce different symbol estimate vectors using the LR-SIC structure. In particular, the first branch

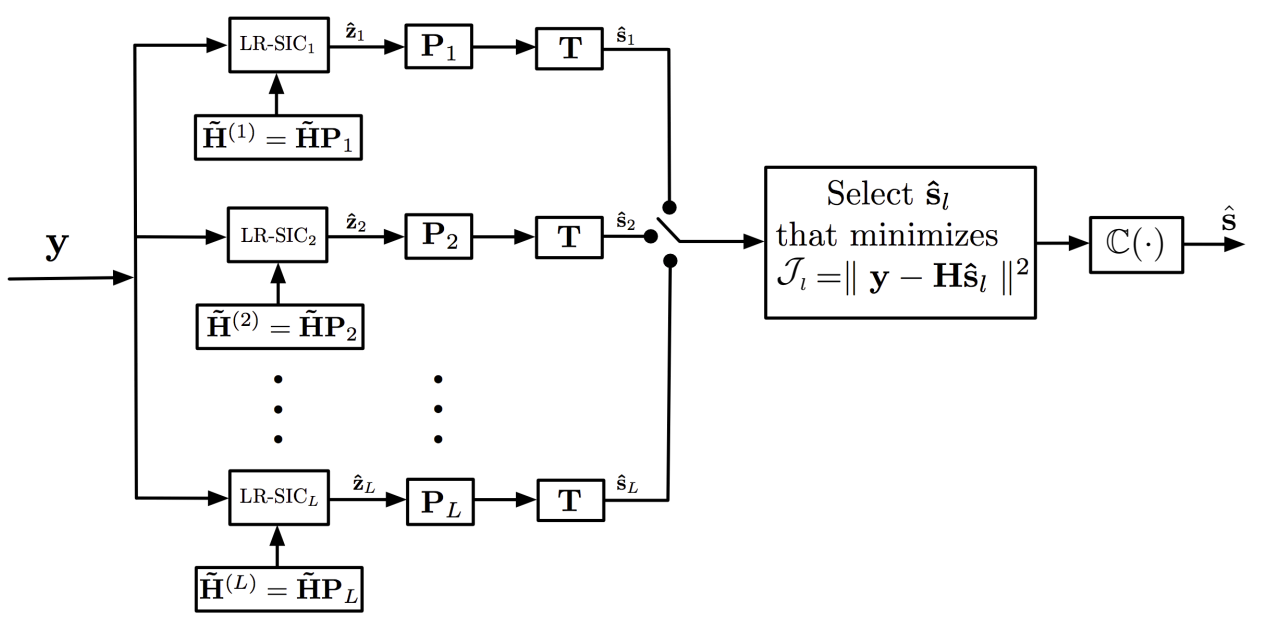

Figure 3.1: Proposed multi-branch lattice reduction successive interference cancellation detection block diagram.

employs a column-norm based ordering in the LR domain and the remaining 
branches use shifted versions of the ordered first branch. The branch with the best performance among the list of candidates is selected using the ML criterion. A schematic of the detector is shown in Figure 3.1. The MB-LR-SIC detector employs an LR transformation in the channel matrix as in (3-5). Let $\mathbf{P}_{l}$ be a column permutation matrix. Then the signal term in (3-5) can be expressed as

$$
\tilde{\mathbf{H}} \mathbf{z}=\tilde{\mathbf{H}} \mathbf{P}_{l} \mathbf{P}_{l}^{T} \mathbf{z}=\tilde{\mathbf{H}}^{(l)} \mathbf{z}_{l},
$$

where $\tilde{\mathbf{H}}^{(l)}=\tilde{\mathbf{H}} \mathbf{P}_{l}=\mathbf{H}\left(\mathbf{T P}_{l}\right)$ is a column permuted version of $\tilde{\mathbf{H}}$ and

$$
\mathbf{z}_{l}=\mathbf{P}_{l}^{T} \mathbf{z}=\left(\mathbf{P}_{l}^{T} \mathbf{T}^{-1}\right) \mathbf{s}
$$

In the proposed scheme, the $l$-th branch $(l=1, \ldots, L)$ of the receiver employs an LR-SIC detector, as described in Section 3.3, based on the permuted matrix $\tilde{\mathbf{H}}^{(l)}=\tilde{\mathbf{H}} \mathbf{P}_{l}$ to generate an estimate $\hat{\mathbf{z}}_{l}$ of $\mathbf{z}_{l}$. Then, the $l$ th branch transmitted signal candidate is obtained by

$$
\hat{\mathbf{s}}_{l}=\left(\mathbf{P}_{l}^{T} \mathbf{T}^{-1}\right)^{-1} \hat{\mathbf{z}}_{l}=\left(\mathbf{T} \mathbf{P}_{l}\right) \hat{\mathbf{z}}_{l}, \quad l=1, \ldots, L .
$$

The best candidate out of the $L$ estimated data signal vectors is selected using the ML criterion, that is

$$
\hat{\mathbf{s}}=\arg \min \left\|\mathbf{y}-\mathbf{H} \hat{\mathbf{s}}_{l}\right\|^{2}, \quad l=1, \ldots, L .
$$

In order to guarantee that a good candidate is always present in the set of

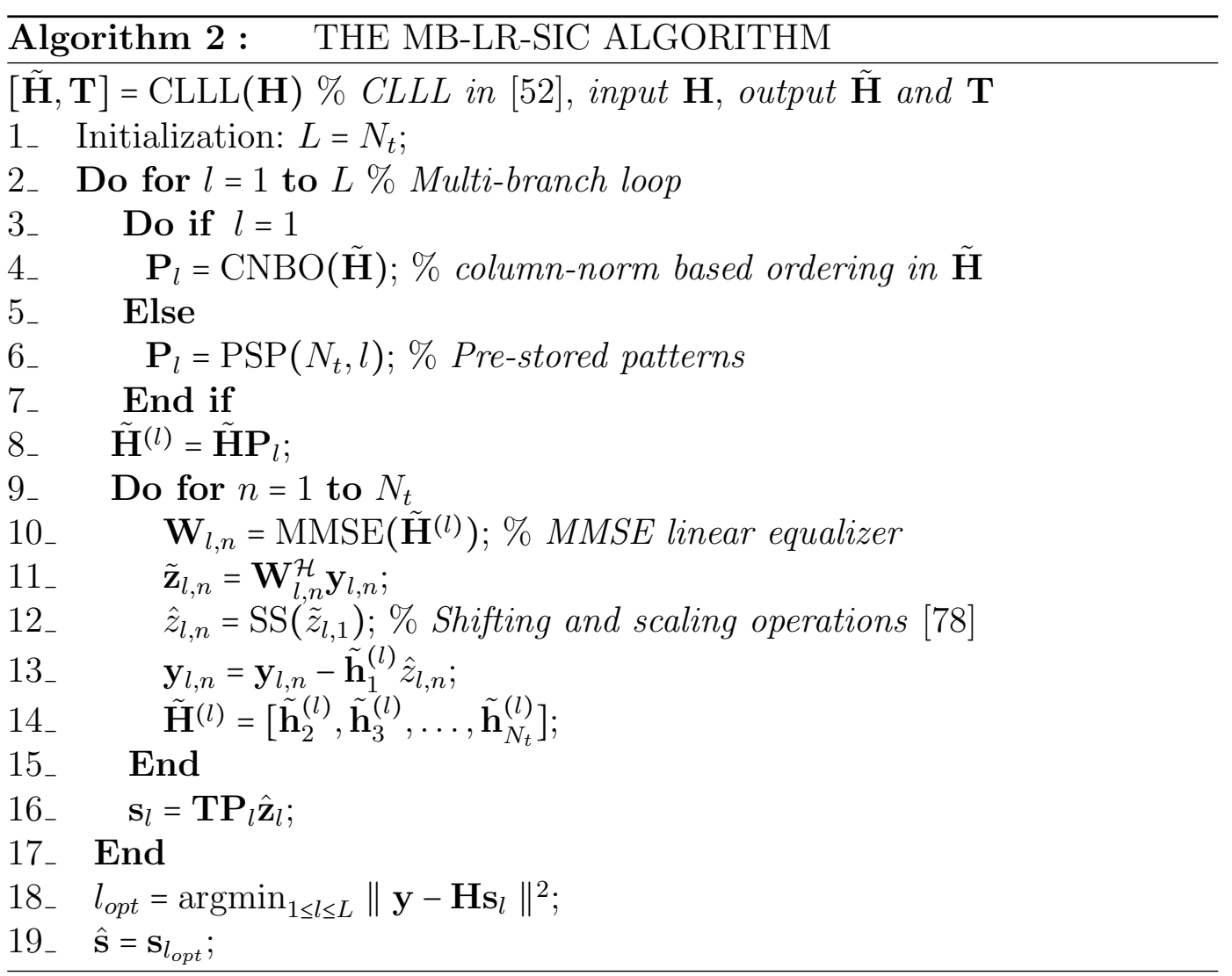


the $L$ candidates generated, one of the branches (e.g. $l=1$ ) should implement a performance effective ordering (e.g. column-norm based ordering) in the LR domain. Here, the remaining branches used the so called Pre-Stored Patterns (PSP) proposed in [75]. The description of the proposed MB-LR-SIC detection structure is depicted in Algorithm 2.

The proposed scheme increases the chances of obtaining more reliable candidates for the transmitted data symbol vector. The MB-LR-SIC scheme shows a high diversity gain and can deliver a performance which, in some cases, is very close to the optimal ML detector. However, due to the fixed number of candidates generated for the MB-LR-SIC, in systems with a large number of antennas, its computational cost could be high.

\section{5}

\section{Variable List Detection}

Some sub-optimal detectors have been reported in the last few years based on list generation, where a list of candidates for each detected symbol is generated [38]. However, for high values of SNR their performances increasingly depart from the ML detector. On the other hand, it is very difficult to generate a list of candidates for a specific symbol in the LR domain due to the fact that the components of the vector $\mathbf{z}=\mathbf{T}^{-1} \mathbf{s}$ in (3-5) are not mutually independent. Also since $\mathbf{T}$ is channel dependent, the set $\tilde{\mathcal{A}}=\mathbf{T}^{-1} \mathcal{A}$ of possible values of $\mathbf{z}$ will change for each realization of the channel matrix $\mathbf{H}$ and its size could be very large if the system has many antennas. In this section, we describe the proposed VLD scheme which allows one to reduce the high SNR performance gap between the LR based detector and the ML detector. The proposed scheme forms a list of candidates as potential solutions for the transmitted symbol vector in the LR domain, using different ordering patterns for the LR-SIC structure. Since the performance of the LR-SIC detector depends strongly on the ordering of the symbols being detected, VLD makes a multistage search for a good ordering pattern that can offer the minimum ML cost, using a starting stage with an ordering based on the MSE values of the estimated symbols in the LR domain. Different from other detection schemes like the MB-LR-SIC detector [69], proposed in Section 3.4, where a fixed number of candidates are tested, the proposed scheme tests a variable size list of candidates, whose length depends on the estimation quality, seeking to find an efficient solution without having to test an unnecessarily large number of candidates. VLD searches among the $N_{t}$ ! -1 possible patterns, an ordering pattern that offers a better performance than the actual ordering, based on an update rule that considers the ML cost of the current candidate as well as its first and second-order 
moments for an error free decision. The decision vector and the number of candidates to be tested are updated in each stage. Before the multistage search for an efficient ordering, VLD computes the starting candidate vector $\mathbf{z}^{0}$. To reduce the number of candidates, a good starting point is convenient. The error covariance matrix between the symbol vector $\mathbf{z}$ and its estimate before the shifting and scaling operation $\tilde{\mathbf{z}}$ is given by $\Phi \triangleq \mathbb{E}\left[(\mathbf{z}-\tilde{\mathbf{z}})(\mathbf{z}-\tilde{\mathbf{z}})^{\mathcal{H}}\right]$. For the LR-SIC detector in (3-25), it can be shown [50] [55] that the error covariance matrix $\Phi_{n}$ at the $n$-th detection layer can be calculated as

$$
\frac{1}{\sigma_{n}^{2}} \Phi_{n}=\bar{\Phi}_{n}=\left(\left[\begin{array}{c}
\tilde{\mathbf{H}}_{[n]} \\
\sigma \mathbf{T}_{[n]}
\end{array}\right]^{\mathcal{H}}\left[\begin{array}{c}
\tilde{\mathbf{H}}_{[n]} \\
\sigma \mathbf{T}_{[n]}
\end{array}\right]\right)^{-1}
$$

Then the optimal, in the MSE sense, symbol detection ordering for the LRSIC is implemented by choosing at each detection layer the symbol index corresponding to the smallest diagonal value of the matrix $\bar{\Phi}_{n}$. The MSE based ordering is used to define a column permutation matrix $\mathbf{P}^{0}$, then the signal term in (3-5) can be expressed as

$$
\tilde{\mathbf{H}} \mathbf{z}=\tilde{\mathbf{H}} \mathbf{P}^{0}\left(\mathbf{P}^{0}\right)^{T} \mathbf{z}=\tilde{\mathbf{H}}^{0} \mathbf{z}_{0},
$$

where $\tilde{\mathbf{H}}^{0}=\tilde{\mathbf{H}} \mathbf{P}^{0}$ is a column permuted version of $\tilde{\mathbf{H}}$ using the MSE based ordering and

$$
\mathbf{z}_{0}=\left(\mathbf{P}^{0}\right)^{T} \mathbf{z}=\left(\mathbf{P}^{0}\right)^{T}\left(\mathbf{T}^{-1} \mathbf{s}\right) .
$$

To compute an estimate $\hat{\mathbf{z}}_{0}$ of $\mathbf{z}_{0}$, the receiver employs an LR-SIC detector, as described in (3-25), based on the permuted matrix $\tilde{\mathbf{H}}^{0}=\tilde{\mathbf{H}}_{e} \mathbf{P}^{0}$ followed by the quantization procedure (3-22). The order of the elements of the estimated vector is rearranged to the original order, yielding the final estimate:

$$
\mathbf{z}^{0}=\mathbf{P}^{0} \hat{\mathbf{z}}_{0}
$$

After computing the starting candidate vector $\mathbf{z}^{0}$, VLD makes a measure of the estimation quality of $\mathbf{z}^{0}$ and, based on it, estimates the number of candidates that are needed to find a better solution. We can equivalently obtain the ML estimate of the information vector $\mathbf{s}$ in (2-2) as:

$$
\begin{aligned}
\hat{\mathbf{s}}_{m l} & =\arg \min _{\mathbf{s} \in \mathcal{A}^{N_{t}}}\|\mathbf{y}-\mathbf{H} \mathbf{s}\|^{2} \\
& =\arg \min _{\mathbf{s} \in \mathcal{A}^{N_{t}}}\left\|\mathbf{y}-\mathbf{H} \mathbf{T} \mathbf{T}^{-1} \mathbf{s}\right\|^{2} \\
& =\mathbf{T} \arg \min _{\mathbf{z} \in \tilde{\mathcal{A}}^{N_{t}}}\|\mathbf{y}-\tilde{\mathbf{H}} \mathbf{z}\|^{2} .
\end{aligned}
$$

As we known, it is not practical to compute the alphabet $\tilde{\mathcal{A}}$, however, if we have a number of candidates as potential solutions for the transmitted signal vector in the LR domain, we can use the minimization in (3-34) before the 
pre-multiplication by the uni-modular matrix $\mathbf{T}$. Then, the quality metric can be evaluated in the LR domain through the ML cost:

$$
\operatorname{ML}-\operatorname{LR}\left(\mathbf{z}^{0}\right)=\left\|\mathbf{y}-\tilde{\mathbf{H}} \mathbf{z}^{0}\right\|^{2}
$$

and the ML cost of an error-free decision, scaled by its standard deviation, named standardized ML cost. Note that, using (3-35) it is not necessary to transform the estimate $\mathbf{z}^{0}$ from the LR domain to the constellation domain which reduces the algorithm's computational complexity. The ML cost of an error-free detection corresponds to $\|\mathbf{n}\|^{2}$, which is Chi-squared distributed with $2 N_{r}$ degrees of freedom with mean $N_{r} \sigma_{n}^{2}$ and variance $N_{r} \sigma_{n}^{4}$. The standardized ML cost of the current candidate is given by

$$
\phi\left(\mathbf{z}^{0}\right)=\frac{\operatorname{ML}-\operatorname{LR}\left(\mathbf{z}^{0}\right)-N_{r} \sigma_{n}^{2}}{\sqrt{N_{r}} \sigma_{n}^{2}} .
$$

Then, the number of candidates $L$ needed to stop the algorithm is determined by an increasing function of $\phi\left(\mathbf{z}^{0}\right)$ and a lower-bound hard limit $L_{m i n}$, where $L_{\text {min }}$ is the minimum number of candidates to be tested after each stage. Here the length of the candidate list is calculated by [79] [80]

$$
L=\left\lceil\max \left(c_{1} \phi\left(\mathbf{z}^{\mathbf{0}}\right), L_{\min }\right)\right\rceil,
$$

where $c_{1}$ is a metric parameter and $\lceil\cdot\rceil$ stands for the smallest integer greater than the argument. To avoid the generation of an excessive number of stages, VLD uses the quality measure $\phi\left(\mathbf{z}^{0}\right)$ to determine the number of candidates, $L$, that needs to be tested in the search for an ordering pattern better than the initial MSE ordering. With that, a stopping condition is guaranteed. The algorithm searches for a new solution $\mathbf{z}^{1}$, using the LR-SIC detector, with a randomly chosen cancellation ordering taken from the remaining $N_{t}$ !-2 possible orderings, to construct the column permutation matrix $\mathbf{P}^{1}$. To compute the new estimate vector $\mathbf{z}^{1}$, the procedure in (3-31)-(3-33) is repeated, now with the permutation matrix $\mathbf{P}^{1}$. If the ML-LR cost function of $\mathbf{z}^{1}$ is less than the ML-LR cost function of $\mathbf{z}^{0}$, the current estimate is updated to $\mathbf{z}^{1}$ and the process is repeated with a new number of candidates $L$, calculated based on the new current estimation vector, and so on. If, at any given stage, the required $L$ candidates are tested without finding a new solution, the algorithm sets the current decision as the final one and stops. With the proposed algorithm, the number of candidates in each stage will never increase when compared with the previous stage because the value of $\phi(\cdot)$ is reduced when a better solution is found. Therefore, VLD prevents an unnecessary number of candidates, among all $N$ ! possible, to be tested.

The description of the proposed VLD structure is depicted in Al- 


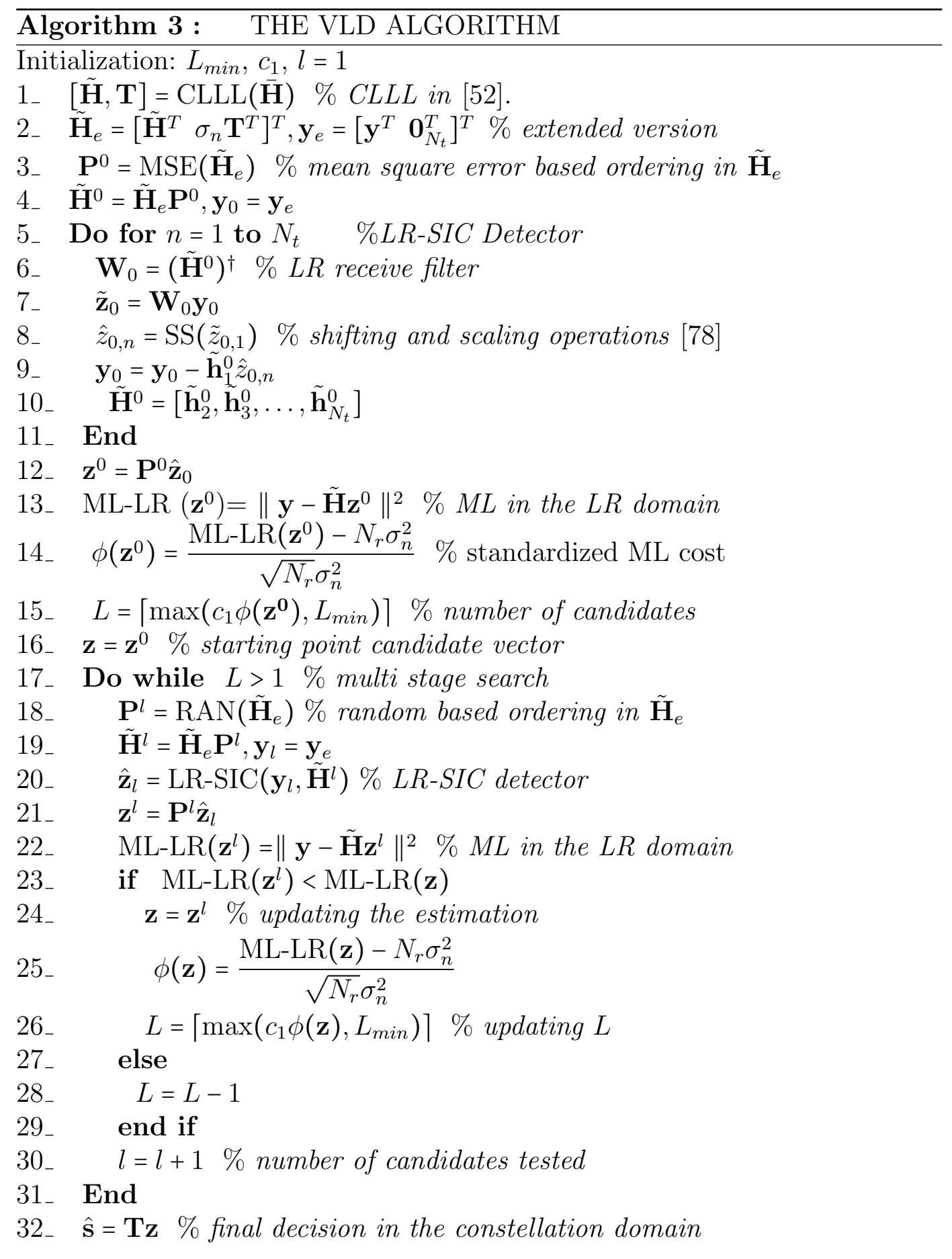


gorithm 3. It is worth noting that the MB-LR-SIC detector and the proposed VLD schemes both reach full diversity gain (Appendix B). However, the performance results obtained by computer simulation, presented in Section 3.6, show that the VLD scheme increases the probability of computing a reliable estimate for the data symbol vector, reducing substantially the performance gap to the ML detector in the high SNR region.

\section{6}

\section{Numerical Results}

In this section, we compare the computational complexity and the BER performance of the proposed MB-LR-SIC and VLD schemes with previously proposed detection algorithms, which include SIC, LR-SIC, MB-SIC, and ML. The SIC detector of [34] uses a norm-based cancellation ordering, the MB-SIC of [37] and the MB-LR-SIC [69] employ a fixed number of branches, equal to the total number of transmit antennas $N_{t}=\sum_{k=1}^{K} N_{t_{k}}$, and norm-based ordering in its first branch. MSE-based ordering is used in the LR-SIC and in the first branch of VLD. All SIC-based detectors use MMSE linear receive filters. A MUMIMO system with $K$ active users is considered. The SNR per transmitted information bit is defined as in (2-82). Two scenarios for the channels associated with each active user are considered. In the first scenario (scenario A), we consider independent and identically distributed random fading channel models whose coefficients are complex Gaussian random variables with zero mean and unit variance. In the second scenario (scenario B) we consider a more realistic channel described by

$$
\mathbf{H}_{k}=\alpha_{k} \beta_{k} \mathbf{G}_{k}, \quad k=1, \ldots, K .
$$

where $\alpha_{k}$ represents the distance based path-loss between the $k$-th transmitter and the receiver, and $\beta_{k}$ is a log-normal variable, representing the shadowing between the transmitter and the receiver. The parameters $\alpha_{k}$ and $\beta_{k}$ are respectively calculated by

and

$$
\alpha_{k}=\sqrt{\frac{L_{p}^{(k)}}{d_{k}^{\tau}}},
$$

$$
\beta_{k}=10^{\frac{\mu_{k} \vartheta_{k}}{10}},
$$

where $L_{p}^{(k)}$ is the base power path loss of the link associated with the $k$ th user, $d_{k}$ is the relative distance between the user and the BS, $\tau$ is the path loss exponent chosen between 2 and 4 depending on the environment, $\vartheta_{k}$ represents a Gaussian distribution with zero mean and unit variance and $\mu_{k}$ is the shadowing spread in $\mathrm{dB}$. The matrix $\mathbf{G}_{k}$ in (3-38) is modeled as the Kronecker channel model [81], expressed by 


$$
\mathbf{G}_{k}=\mathbf{R}_{r_{x}}^{1 / 2} \mathbf{G}_{0_{k}} \mathbf{R}_{t_{x_{k}}}^{1 / 2}
$$

where $\mathbf{G}_{0_{k}}$ is the MIMO channel matrix for scenario $A$ and $\mathbf{R}_{r_{x}}$ and $\mathbf{R}_{t_{x_{k}}}$ denote the receive and transmit correlation matrices, respectively. We assume that $L_{p}^{(k)}=L_{p}, \mu_{k}=\mu$ and the same correlation matrix $\mathbf{R}_{t_{x_{k}}}^{1 / 2}=\mathbf{R}_{t_{x}}^{1 / 2}$ for all $K$ transmitters. The components of the correlation matrices $\mathbf{R}_{r_{x}}$ and $\mathbf{R}_{t_{x_{k}}}$ are of the form:

$$
\mathbf{R}=\left[\begin{array}{ccccc}
1 & \rho & \rho^{4} & \ldots & \rho^{\left(N_{a}-1\right)^{2}} \\
\rho & 1 & \rho & \ldots & \vdots \\
\rho^{4} & \rho & 1 & \vdots & \rho^{4} \\
\vdots & \vdots & \vdots & \vdots & \vdots \\
\rho^{\left(N_{a}-1\right)^{2}} & \ldots & \rho^{4} & \rho & 1
\end{array}\right]
$$

where $N_{a}$ is the number of antennas and $\rho$ is the correlation index of neighboring antennas ( $\rho=\rho_{t_{x}}$ for the transmit antennas and $\rho=\rho_{r_{x}}$ for the receive antennas). Note that $\rho=0$ represents an uncorrelated scenario and $\rho=1 \mathrm{im}$ plies a fully correlated scenario.

\subsection{1}

\section{Computational Complexity}

In this subsection, the computational complexity of the proposed MBLR-SIC and VLD algorithms is evaluated. The LR transformation has a variable complexity, and for this reason it is important to know its average cost by counting the number of floating points (FLOPs). In [82] a reduced and fixed complexity LR algorithm was proposed. The LR transformation is not the main focus in this thesis, for this reason we use the conventional CLLL algorithm. To compute the number of FLOPs (average number in the VLD case) per received vector $\mathbf{y}$ we use the Lightspeed Matlab toolbox [83]. Figure 3.2 shows the computational complexity for the different detection algorithms focused in this work. Note that the computational cost of the VLD scheme tends to grow with increasing SNR. This is so because the quality of the initial candidate improves with an increasing SNR, and the better is the initial candidate larger is the number of candidates that have to be tested to find a better one. The results in Figure 3.2 are for SNR values of $10 \mathrm{~dB}$ and $14 \mathrm{~dB}$. The complexity of the other detectors, is independent of SNR. For the ML detector, we have illustrated both QPSK and 16-QAM modulation, whereas for the other detectors we have considered only QPSK modulation since the computational cost in these detectors does not change significantly with the order of the QAM modulation. The figure compares the required number of FLOPs (average number in the VLD case) versus the number of antennas for $N_{t}=N_{r}$. The proposed MB-LR-SIC has a significant lower 


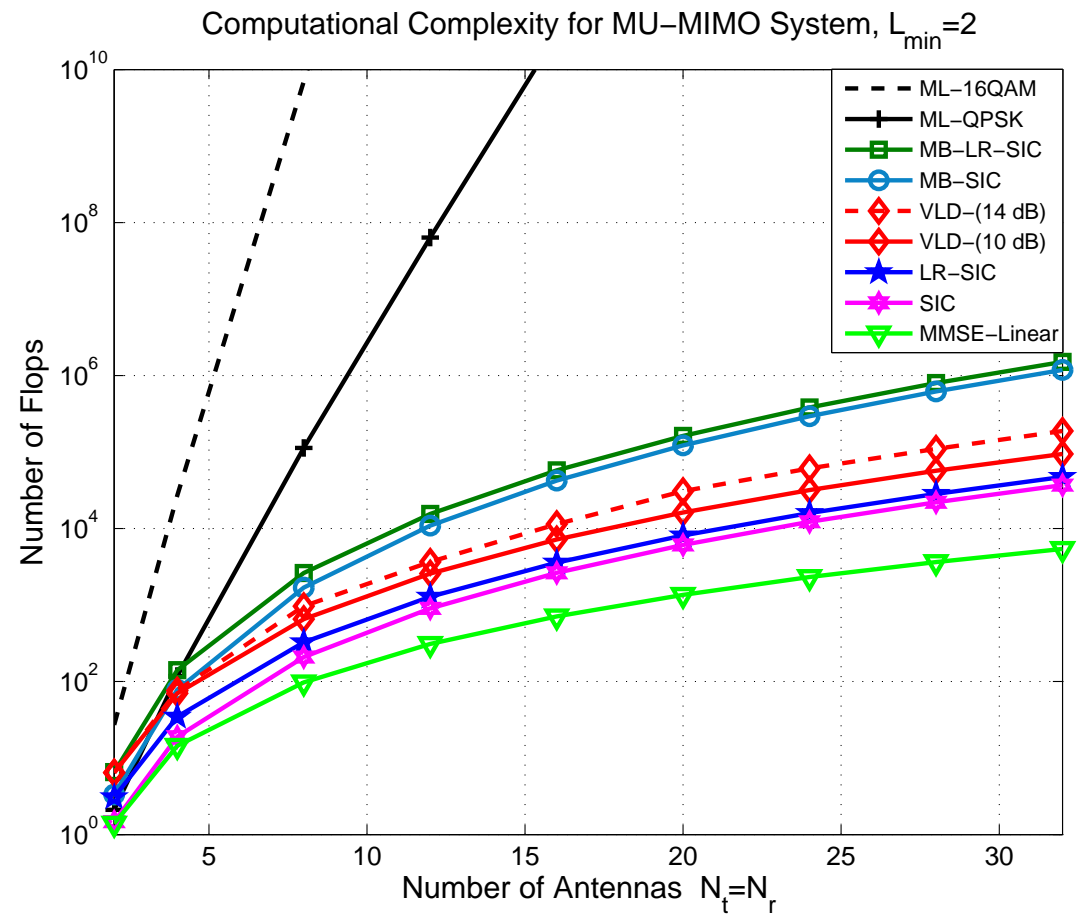

Figure 3.2: Computational complexity of detection algorithms.

complexity when compared with the ML detector and, as will be shown in the next subsection, in some specific scenarios, delivers a performance very close to that of the ML detector. The proposed VLD algorithm has a significantly lower complexity when compared with the MB-LR-SIC and MB-SIC schemes and the ML detector and a performance close to that of the ML detector, as will be shown in the sequence. The computational cost of VLD algorithm, depends on the computation of the reduced basis of the channel matrix and the search for an efficient ordering for the LR-SIC detector. In quasi static channels the cost of the LR transformation is not a major concern, because the lattice is fixed during a long period of time and the basis is stored for subsequent use. VLD offers a flexible control of complexity by varying the parameters $c_{1}$ and $L_{\text {min }}$

\subsection{2}

\section{Performance Results}

In this subsection the BER performances of the MB-LR-SIC and VLD schemes are tested. The numerical results correspond to an average of 3,000 simulations runs, with $500 N_{t}$ symbols transmitted per run.

In Figure 3.3 and Figure 3.4, we consider scenario A and compare the performance of the proposed MB-LR-SIC detector with that of existing detectors, with QPSK and 16-QAM modulation, respectively. In both cases 


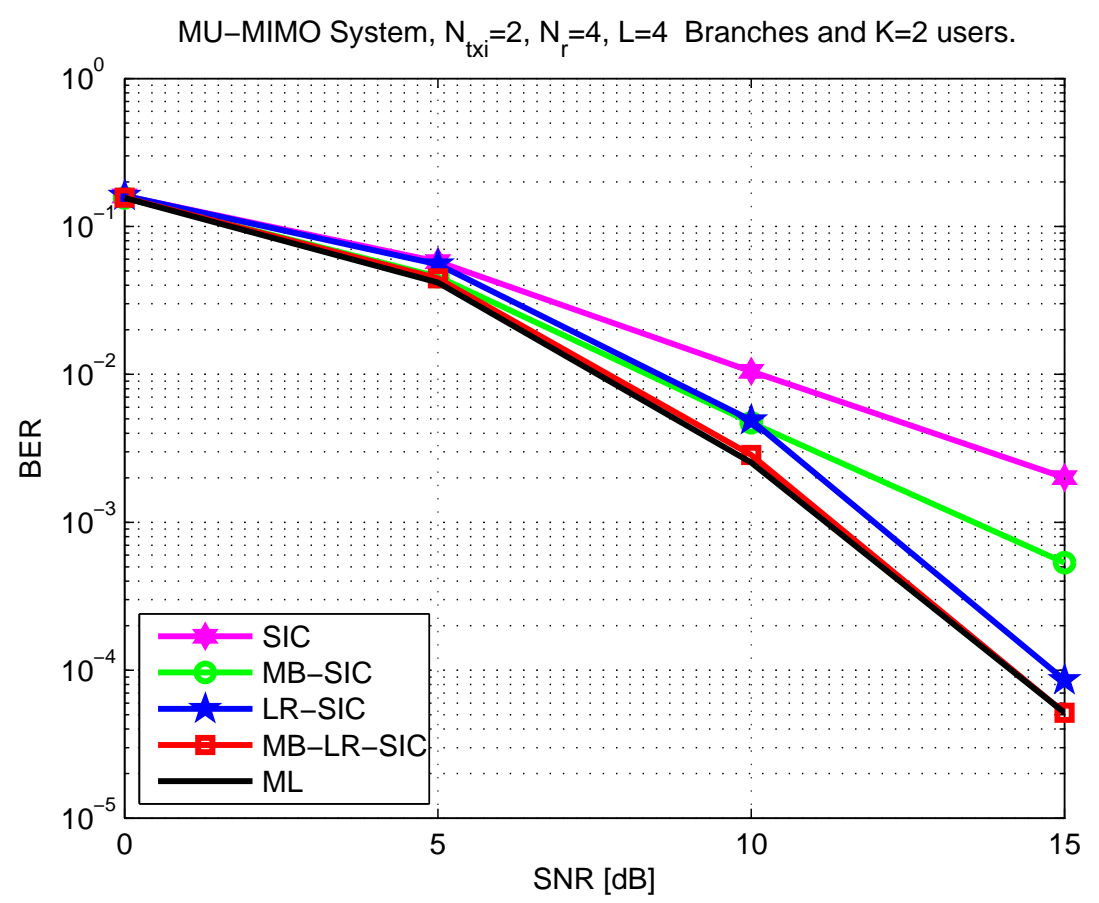

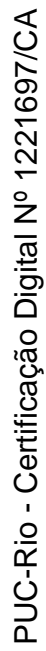

Figure 3.3: BER vs SNR performance for the proposed MB-LR-SIC and existing algorithms in a MU-MIMO scenario. All SIC detectors use a columnnorm-based ordering and QPSK modulation.

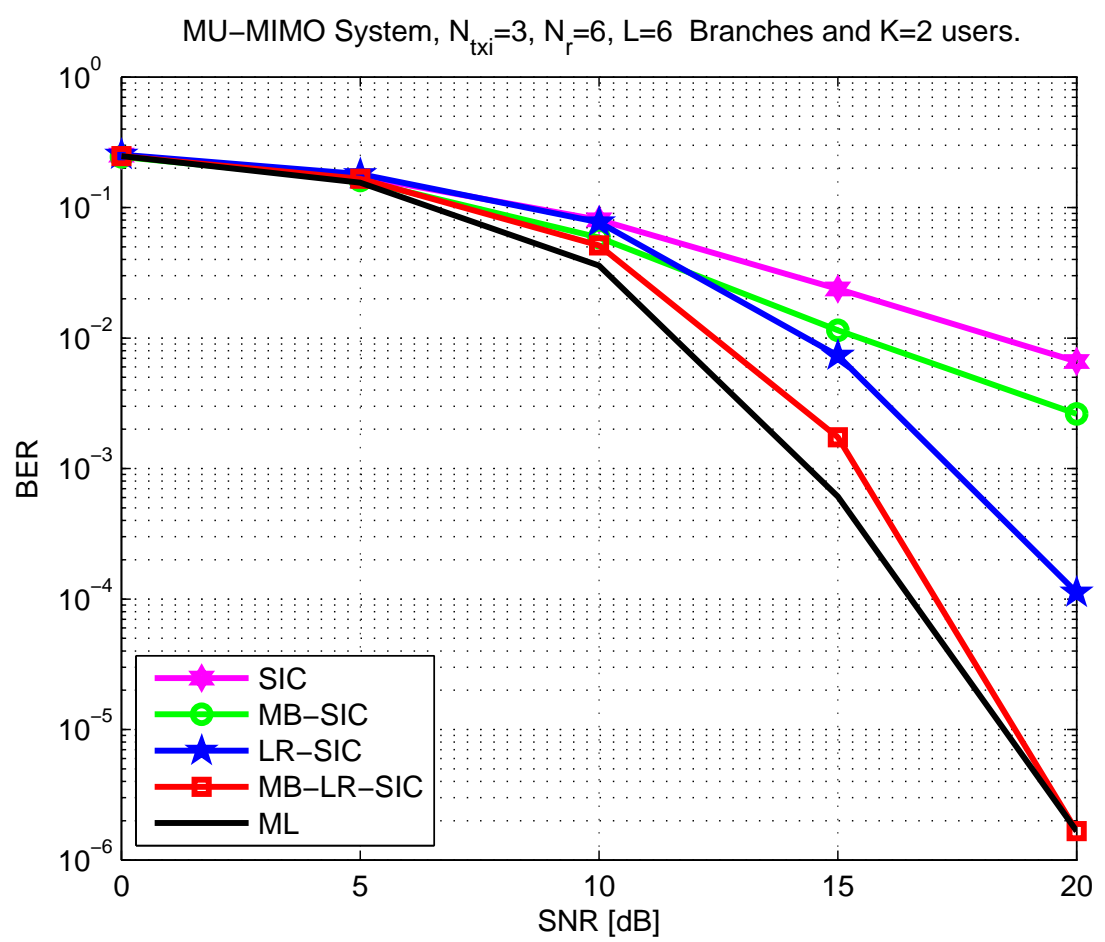

Figure 3.4: BER vs SNR performance for the proposed MB-LR- SIC and existing algorithms in a MU-MIMO scenario. All SIC detectors use a columnnorm-based ordering and 16-QAM modulation. 


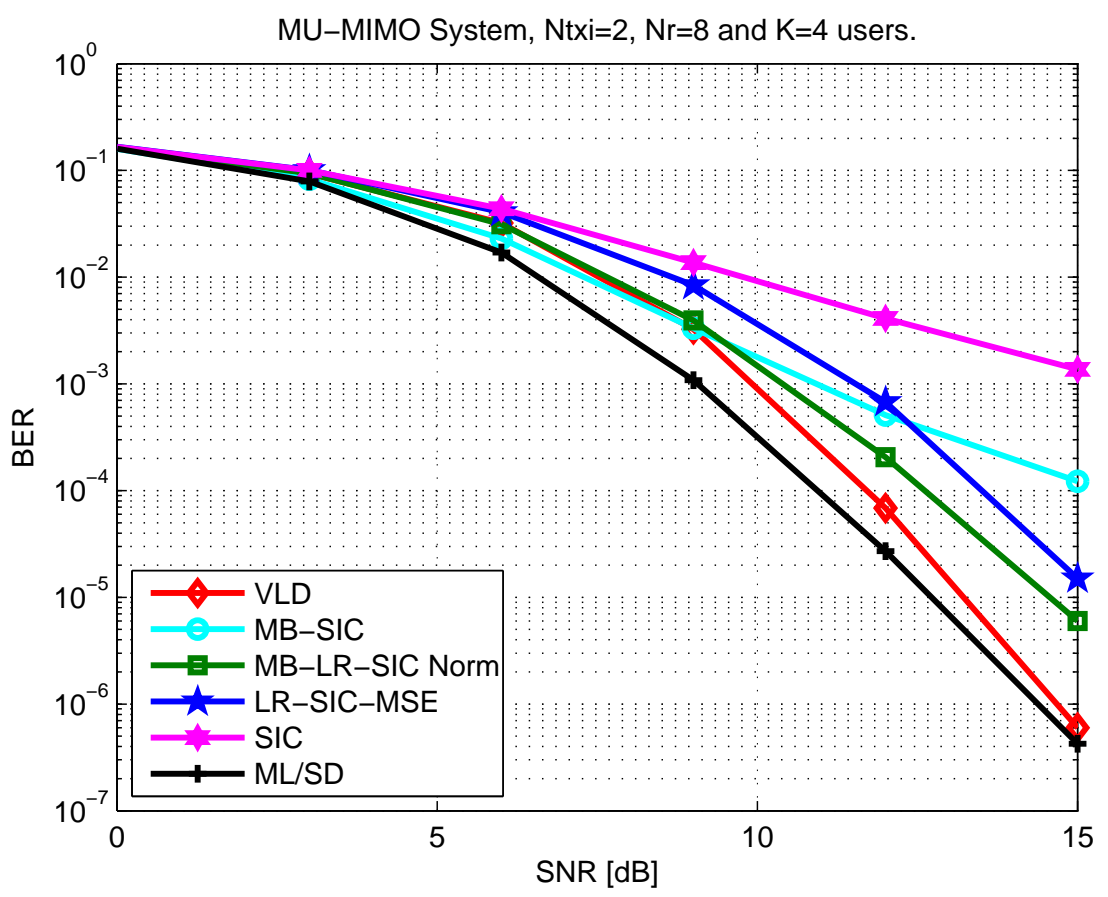

Figure 3.5: BER vs SNR performance for the proposed VLD $\left(L_{\min }=2, c_{1}=2.5\right)$ and existing algorithms with QPSK modulation in scenario A.

we consider $N_{t_{i}}=2$ antenna elements at the user devices. We also consider $K=2$ and $K=3$ active users, $N_{r}=4$ and $N_{r}=6$ receive antennas at the BS, respectively. We consider perfectly known channel state information. Note that the MB-LR-SIC detector, besides being conceptually simple, has been shown through simulations that the performance, in these low number of antennas scenario, approaches the optimal ML detector with much lower computational complexity. However, as it will be shown in the subsequent experiments, the performance of the MB-LR-SIC detector is considerably reduced when the number of antennas increase.

In Figure 3.5, we consider scenario A with $K=4$ active users, $N_{t_{i}}=2$ antenna elements at the user devices and $N_{r}=8$ receive antennas at the BS. We also consider QPSK modulation and perfect channel estimation. For the VLD scheme, we use $L_{\text {min }}=2$ and $c_{1}=2.5$. Note that the performance of the proposed VLD algorithm is close that of the ML detector and the difference between their performances decreases significantly for high values of $S N R$. For the curves in Figure 3.6, the same system parameters of the previous experiment are used, however, a high correlated channel scenario where the transmit and receive correlation coefficients are equal to $\rho=0.75$, $L_{p}=0.7$ and $\tau=2$ is considered. The relative distance $d_{k}$ to the $\mathrm{BS}$ is obtained from a uniform discrete random variable between 0.1 and 0.95 and 


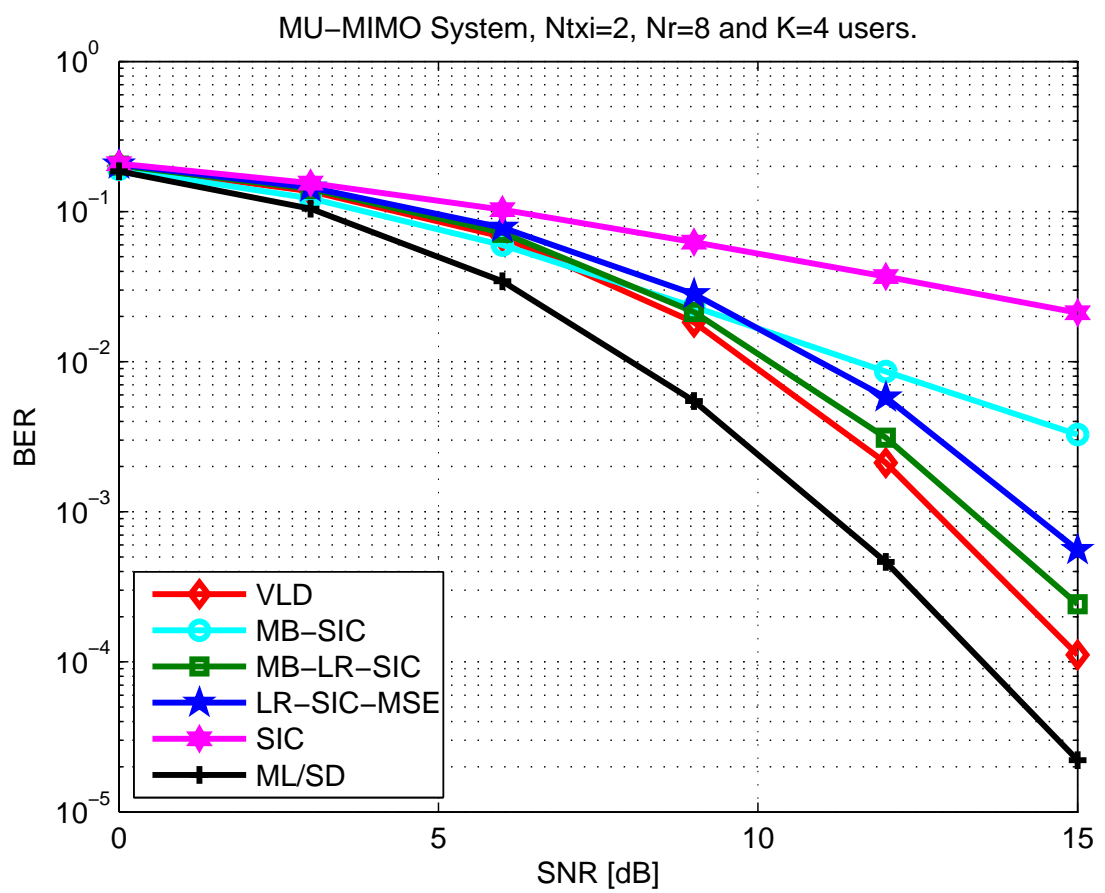

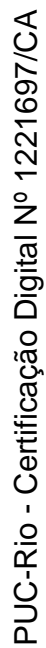

Figure 3.6: BER vs SNR performance for the proposed VLD $\left(L_{\min }=2, c_{1}=2.5\right)$ and existing algorithms with QPSK modulation in scenario B $\left(L_{p}=0.7, \mu=\right.$ $\left.3 \mathrm{~dB}, \rho_{t x}=\rho_{r x}=0.75\right)$.

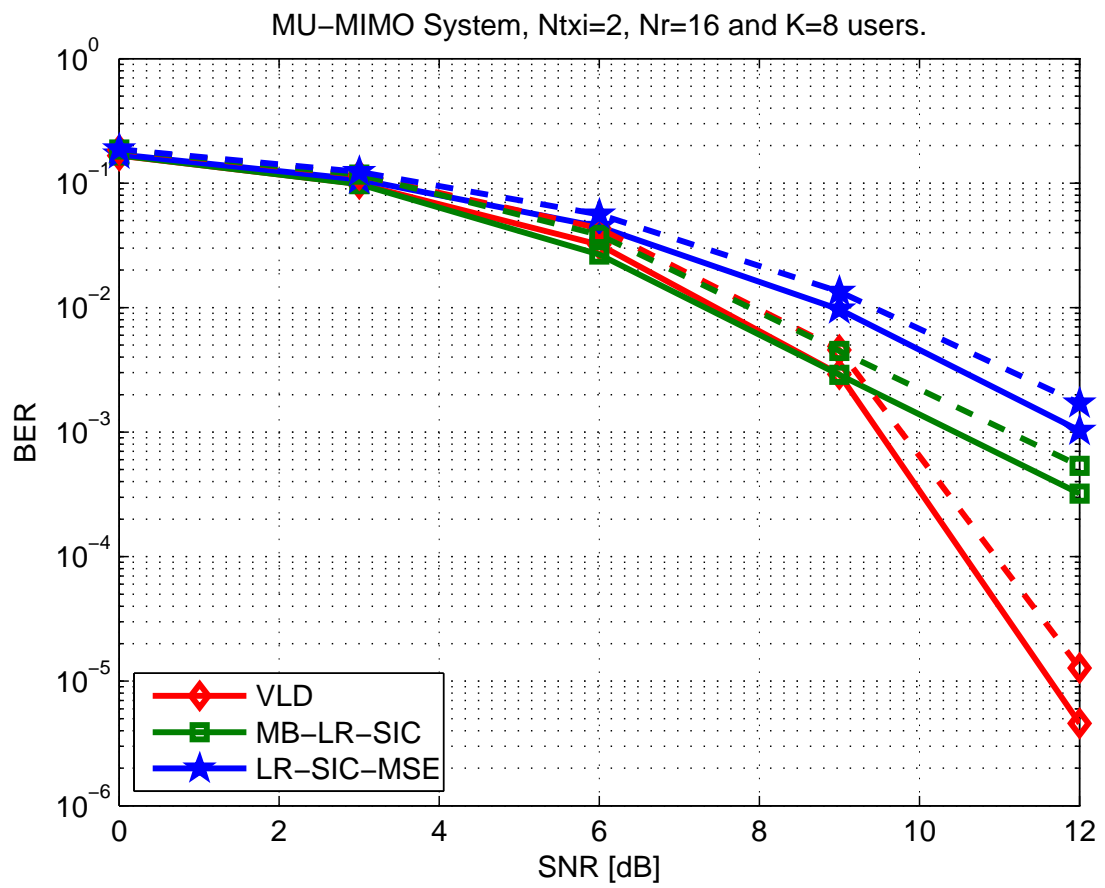

Figure 3.7: BER vs SNR performance for the proposed VLD $\left(L_{\min }=2, c_{1}=5\right)$ and existing algorithms with QPSK modulation in scenario A, LS channel estimation (- -) and perfect channel estimation (-). 


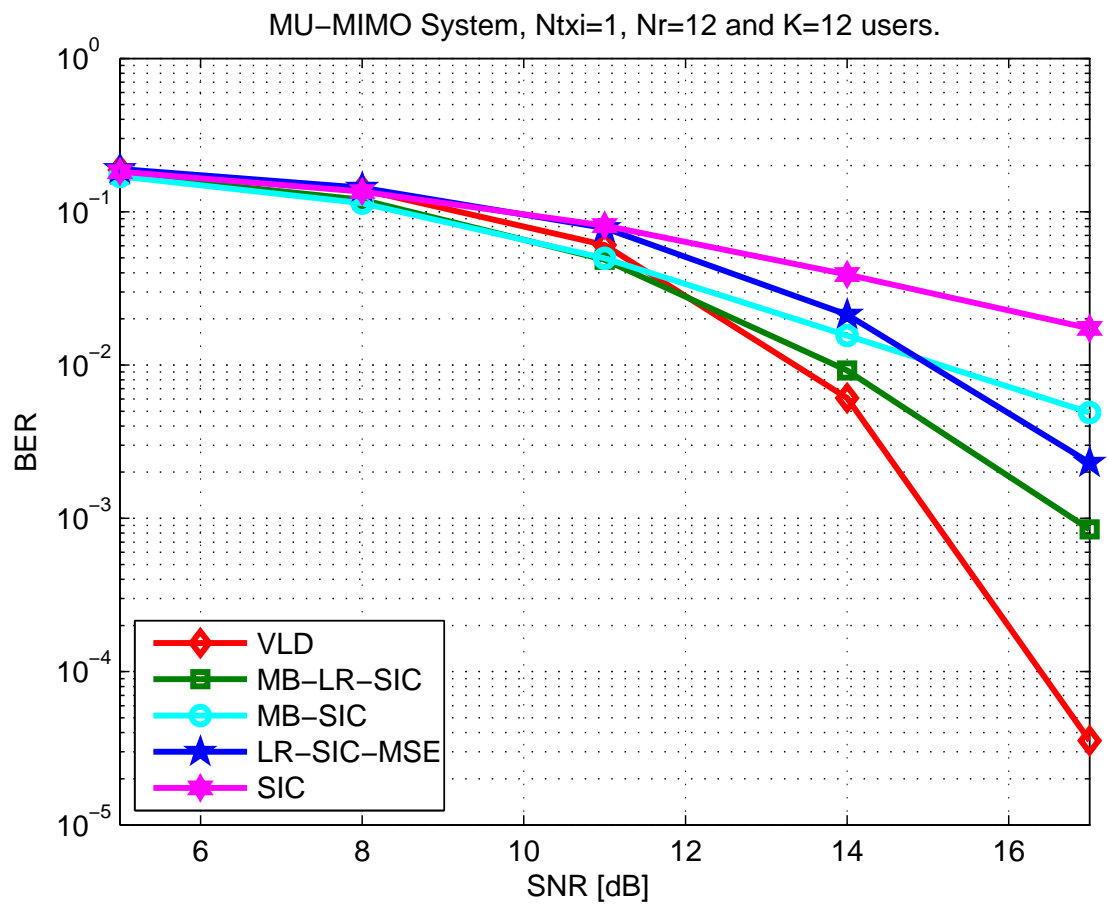

(a)

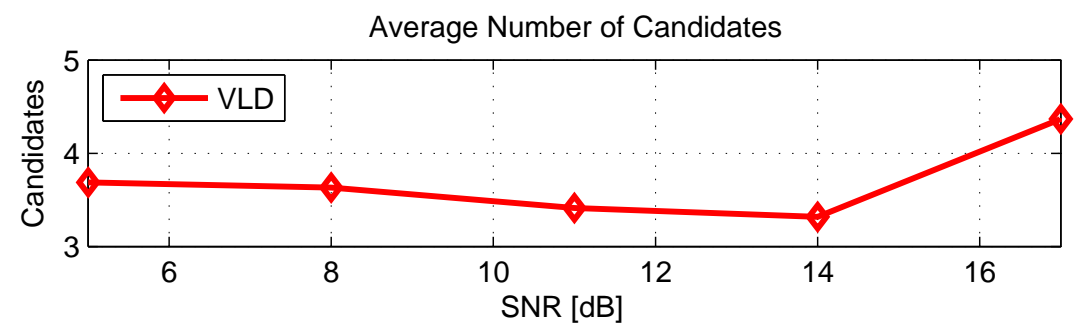

(b)

Figure 3.8: (a) BER vs SNR performance for the proposed VLD $\left(L_{\min }=3, c_{1}=\right.$ 5) and existing algorithms with 16-QAM modulation in scenario A. (b) Average number of candidates tested by VLD vs $\operatorname{SNR}\left(N_{t}=N_{r}=12\right)$.

the shadowing spread is $\mu=3 \mathrm{~dB}$. Due to the propagation and correlation effects, the curves in Figure 3.6 illustrates a loss of performance in all detectors. However, the proposed VLD scheme for this realistic conditions presents a better performance than the other detection schemes and is close to the optimal ML detector.

In the next example, scenario A, QPSK modulation and the least square (LS) channel estimation for different detectors are considered, $600 N_{t}$ symbols are transmitted, where 100 symbols are used for training which are known at the receiver. We employ a forgetting factor equal to 0.998 , consider a system with $K=8$ active users with $N_{t_{i}}=2$ antennas per user and $N_{r}=16$ antennas at BS. The parameters for VLD are $L_{\text {min }}=2$ and $c_{1}=5$. The results in Figure 3.7 evidence that the loss of performance caused by the use of estimated channels is not significant and that the performance of the VLD scheme with imperfect 


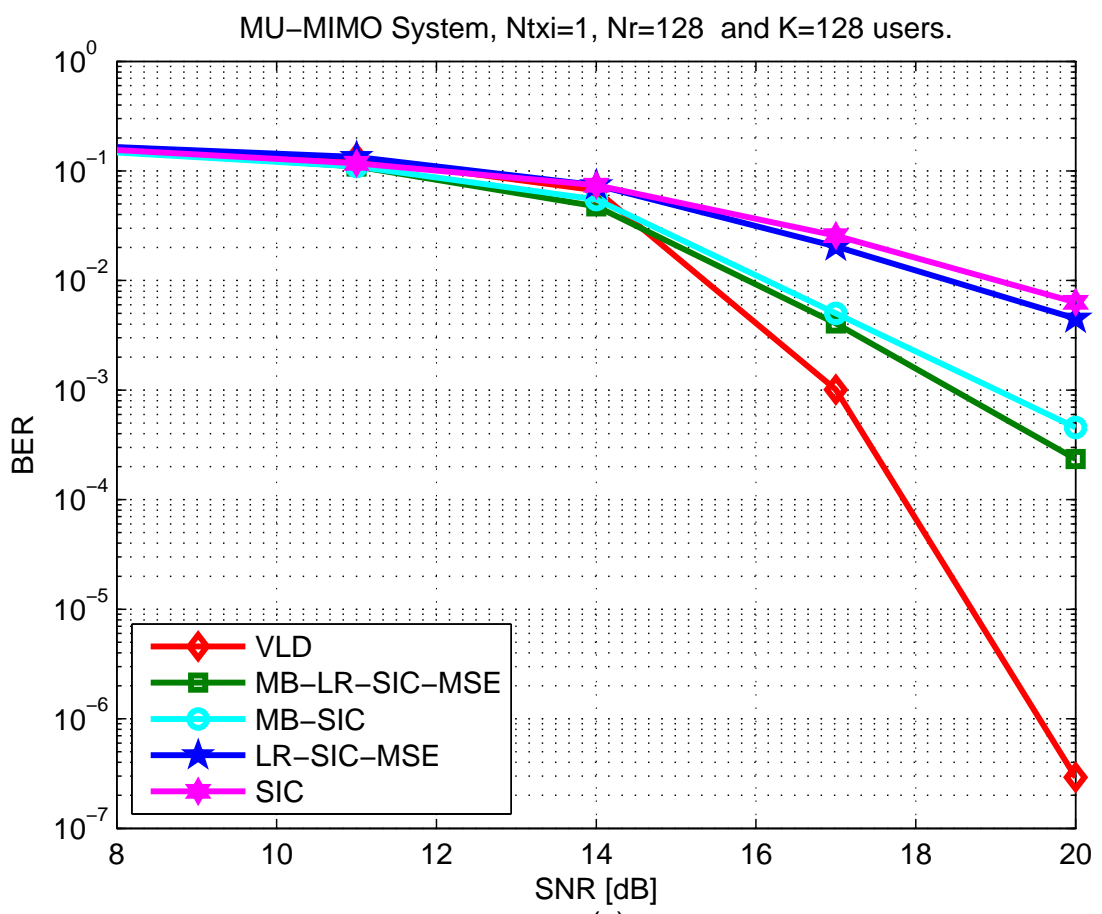

(a)

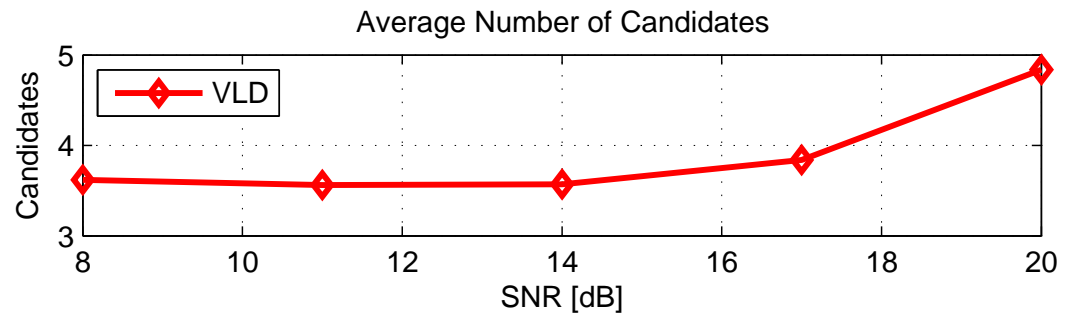

(b)

Figure 3.9: (a) BER vs SNR performance for the proposed VLD $\left(L_{\min }=\right.$ $\left.3, c_{1}=40\right)$ and existing algorithms with 16-QAM modulation in scenario A. (b) Average number of candidates tested by $\operatorname{VLD}$ vs $\operatorname{SNR}\left(N_{t}=N_{r}=128\right)$.

channel estimation (dotted lines) offer a superior BER performance to the MB-LR-SIC and the LR-SIC detectors.

For the experiments in Figure 3.8 and Figure 3.9, we consider scenario A with $K=12$ and $K=128$ user devices, respectively, where each user is equipped with $N_{t_{i}}=1$ transmit antenna. $N_{r}=12$ and $N_{r}=128$ antennas at the BS are also considered, respectively. The systems employ perfect channel state information and 16-QAM modulation. For the VLD algorithm, the parameter chosen are $L_{\min }=3$, in both cases with $c_{1}=5$ and $c_{1}=40$, respectively. Due to the low performance shown by the norm-based ordering for systems with large number of antennas, MSE-based ordering was implemented in the first branch of the MB-LR-SIC detector in Figure 3.9-(a). The results in Figure 3.8(a) and Figure 3.9-(a) indicate a remarkable superiority in performance of the VLD scheme over the others sub-optimal detectors considered in the 
experiments. For high values of SNR and magnitude of the systems, the difference in performance of VLD and MB-LR-SIC increases. In Figure 3.8(b) and Figure 3.9-(b) the average number of candidates tested by VLD along the SNR values is presented. We can see that the number of candidates tested by the proposed scheme is much lower than the MB-LR-SIC which considers a fixed number of candidates for the transmitted symbol vector. It is worth noting that the average number of candidates tested by the VLD scheme tends to grow with increasing SNR, and that makes sense; the quality of the initial candidate improves with a increasing SNR, and the better is the initial candidate, larger is, in the average, the number of candidates that have to be tested before finding a better one, and so being able to improve the detector performance in the high SNR region.

Due to the higher detection diversity and low computational complexity, compared with the optimal detector, the VLD structure is presented as a viable detection alternative for future MIMO communications systems. 


\section{4 \\ Iterative Detection and Decoding for the Uplink of Multiuser MIMO Systems}

\section{1}

\section{Overview}

The iterative detection and decoding (IDD) receiver, based on the turbo principle [84], can improve the performance of MIMO systems [56]. Similarly to uncoded system, where the ML detector is not a viable solution, for coded systems the maximum a posteriori probability (MAP) detector is desirable but the complexity grows with the number of transmit antennas and the constellation size. For this reason, computational efficient detectors for coded systems are necessary.

In this chapter, we propose a detection scheme for the uplink of MUMIMO systems which combines a IDD receiver with convolutional codes and the previously proposed VLD algorithm.

The chapter is organized as follows. Section 4.2 examines the system model for Point-to-Point MIMO system with IDD detection. In Section 4.3 the proposed iterative VLD and decoding scheme for MU-MIMO system is presented. Simulation results are presented and discussed in Section 4.4

\section{2}

\section{Iterative Detection and Decoding for Point-to-Point MIMO Systems}

In Figure. 4.2, we show the block diagram of a IDD transmission system for point-to-point MIMO environments. In the transmitter side the information binary sequence $\mathcal{C}$ is encoded by a channel encoded with an $R_{c}$ code rate. The encoder data is reordered by an interleaver to reduce the effect of burst errors. After the interleaver, the coded bits are modulated using a $2^{M}$-ary complex constellation to generate the transmitted signal vector $\mathbf{s}$ which consists of $N_{t} M$ coded bits. The $n$-th symbol of $\mathbf{s}$, transmitted by the $n$-th antenna, is $s_{n}=\left\{b_{n, 1}, b_{n, 2}, \ldots, b_{n, M}\right\}$ where $b_{n, l} \in\{ \pm 1\}$ denotes the $l$-th bit of $s_{n}$. It is possible to assume that the coded bits of each symbol in $\mathbf{s}$ are independent due to the bit interleaver. The IDD receiver consists of two stages separated by interleavers and deinterleavers. The MIMO detector provides the soft decisions of the coded bits to the soft-input soft-output (SISO) channel decoder and the SISO channel decoder provides the MIMO detector with extrinsic bit information which is then used by the MIMO detector in subsequent 


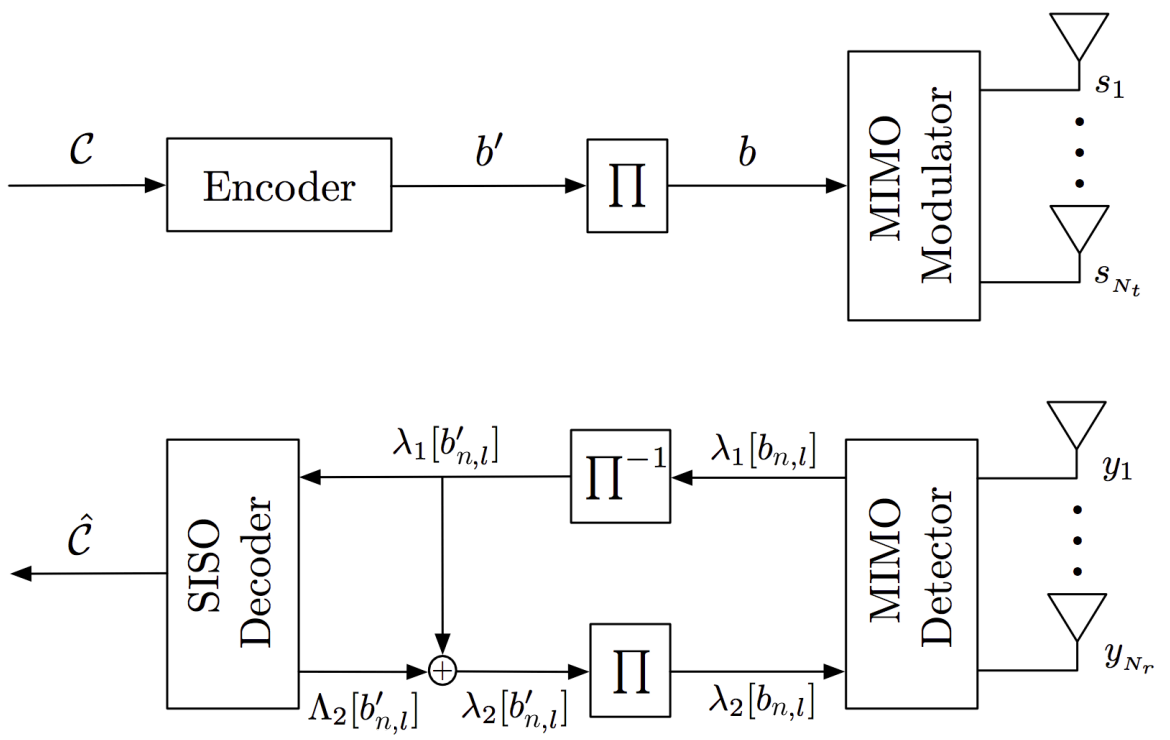

Figure 4.1: Block diagram of a IDD transmission system over a Point-to-Point MIMO channel.

iterations. The IDD receiver improves the performance using the soft bit extrinsic information exchanged between the detector and decoder through iterations.

In the maximum a posteriori probability (MAP) detector, the output soft bit information is computed as the a posteriori log-likelihood ratio (LLR) of $b_{n, l}$, defined as

$$
\Lambda_{1}\left[b_{n, l}\right]=\log \frac{P\left(b_{n, l}=+1 \mid \mathbf{y}\right)}{P\left(b_{n, l}=-1 \mid \mathbf{y}\right)} .
$$

If we use the SIC detection technique, we set the vector $\mathbf{u}=\mathbf{s}+\mathbf{v}_{\text {eff }}$, where $\mathbf{v}_{\text {eff }}$ is the effective noise factor after the MMSE detection in each layer. This assumption provides an efficient accurate way to compute the extrinsic information [85-87]. Considering (4-1) and that the symbol $u_{n}=s_{n}+v_{\text {eff }}$ is statistically independent from the other symbols [88], we have

$$
\Lambda_{1}\left[b_{n, l}\right]=\log \frac{P\left(b_{n, l}=+1 \mid \mathbf{u}\right)}{P\left(b_{n, l}=-1 \mid \mathbf{u}\right)} \approx \log \frac{P\left(b_{n, l}=+1 \mid u_{n}\right)}{P\left(b_{n, l}=-1 \mid u_{n}\right)} .
$$

Using Bayes's rule, equation (4-2) can be rewritten as

$$
\begin{aligned}
\Lambda_{1}\left[b_{n, l}\right] & =\log \frac{p\left(u_{n} \mid b_{n, l}=+1\right)}{p\left(u_{n} \mid b_{n, l}=-1\right)}+\log \frac{P\left(b_{n, l}=+1\right)}{P\left(b_{n, l}=-1\right)} \\
& =\lambda_{1}\left[b_{n, l}\right]+\lambda_{2}^{p}\left[b_{n, l}\right],
\end{aligned}
$$

the term $\lambda_{2}^{p}\left[b_{n, l}\right]$ represents the a priori information for the coded bit $b_{n, l}$ which 
is provided by the SISO decoder in the previous iteration. The superscript $p$ denotes the value obtained from the previous iteration. For the first iteration we assume $\lambda_{2}^{p}\left[b_{n, l}\right]=0$ for all bits. The term $\lambda_{1}\left[b_{n, l}\right]$ denotes the extrinsic information which is computed based on the received signal vector $\mathbf{y}$ and a priori information of the coded bits except the $l$-th bit, i.e., $\lambda_{2}^{p}\left[b_{n, j}\right]$, where $j \neq l$. For the detector, the coded bit extrinsic LLR for the $n$-th symbol is obtained as

$$
\lambda_{1}\left[b_{n, l}\right]=\log \frac{\sum_{a_{c} \in \mathcal{A}_{n, l}^{+}} p\left(u_{n} \mid s_{n}=a_{c}\right) \exp \left(L_{a}\left(a_{c}\right)\right)}{\sum_{a_{c} \in \mathcal{A}_{n, l}^{-}} p\left(u_{n} \mid s_{n}=a_{c}\right) \exp \left(L_{a}\left(a_{c}\right)\right)},
$$

where $\mathcal{A}_{n, j}^{ \pm}$denotes the sub sets of $\mathcal{A}^{N t}$ whose $l$-th bit of $s_{n}$ is $\pm 1 . L_{a}\left(a_{c}\right)$ denotes the a priori symbol probability for symbol $a_{c}$. As the vector $\mathbf{v}_{\text {eff }}$ in $\mathbf{u}=\mathbf{s}+\mathbf{v}_{e f f}$ is assumed as a Gaussian vector we have

$$
p\left(u_{n} \mid s_{n}\right)=\frac{1}{\pi \sigma_{\text {eff }}^{2}} \exp \left(\frac{-\left|u_{n}-s_{n}\right|^{2}}{\sigma_{\text {eff }}^{2}}\right),
$$

where $\sigma_{\text {eff }}^{2}$ represents the variance of the effective noise of $u_{n}$. Then, we can rewrite equation (4-4) as

$$
\lambda_{1}\left[b_{n, l}\right]=\log \frac{\sum_{a_{c} \in \mathcal{A}_{n, j}^{+}} \exp \left(\frac{-\left|u_{n}-a_{c}\right|^{2}}{\sigma_{e f f}^{2}}\right) \prod_{j \neq l} P\left(b_{n, j}\right)}{\sum_{a_{c} \in \mathcal{A}_{n, j}^{-}} \exp \left(\frac{-\left|u_{n}-a_{c}\right|^{2}}{\sigma_{e f f}^{2}}\right) \prod_{j \neq l} P\left(b_{n, j}\right)}
$$

where $P\left(b_{n, j}\right)$ is a priori probability of a bit $b_{n, j}$ and obtained by its a priori LLR [87] as

$$
P\left(b_{n, j}\right)=\frac{1}{2}\left[1+b_{n, j} \tanh \left(\frac{1}{2} \lambda_{2}^{p}\left[b_{n, j}\right]\right)\right] .
$$

The $\lambda_{1}\left[b_{n, l}\right]$ is deinterleaved and fed to the SISO decoder as the a priori information. The decoder calculates a posteriori LLR for each coded bit using prior information about all posible symbols and the knowledge about the trellis structure of the code [36] as

$$
\Lambda_{2}\left[b_{n, l}^{\prime}\right]=\lambda_{2}\left[b_{n, l}^{\prime}\right]+\lambda_{1}^{p}\left[b_{n, l}^{\prime}\right]
$$

where the extrinsic information $\lambda_{2}\left[b_{n, l}^{\prime}\right]$ is the information about the code bit $b_{n, l}^{\prime}$ obtained from the prior information about the other code bits $\lambda_{1}^{p}\left[b_{n, j}^{\prime}\right], j \neq l$. The a posteriori LLR of every information bit is also collected by the decoder which is used to make the decision of the message bit at the last iteration. The extrinsic information obtained by the decoder is fed back to the detector as the a priori information. At the first iteration, $\lambda_{1}$ and $\lambda_{2}^{p}$ are statistically independent and as the iterations are performed they become more correlated until the improvement diminishes. 

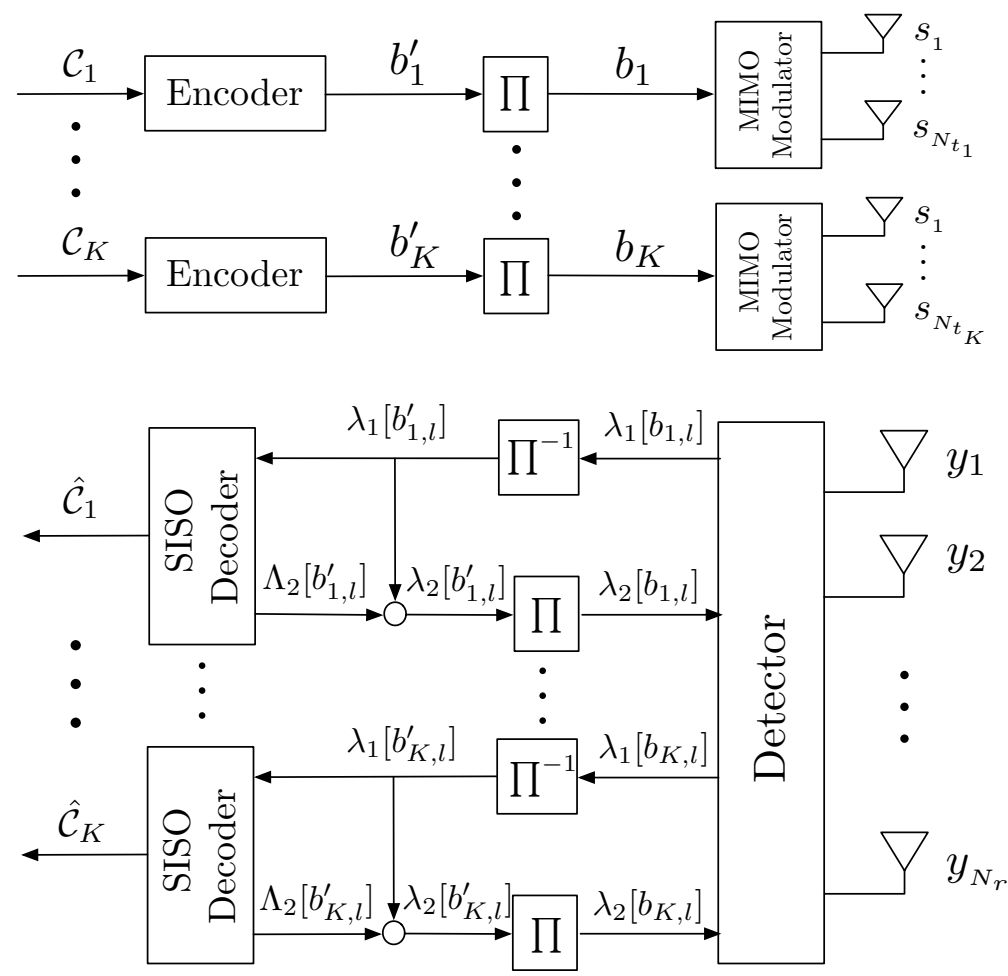

Figure 4.2: IDD receiver for MU-MIMO system.

\section{3}

\section{Iterative Variable List detection and Decoding}

In this section, we present the iterative variable list detection and decoding (IVLDD) scheme for the uplink MU-MIMO system. As depicted in Figure 4.2, we consider $K$ active users, where the $k$-th user, equipped with $N_{t_{k}}$ transmit antennas, employs convolutional codes, with an $R_{c}$ code rate, to encode the information binary sequence $\mathcal{C}_{k}$. The encoded data bit is reordered by an interleaver. After the interleaver, the coded bits are modulated using a $2^{M}$-ary complex constellation to generate the transmitted signal vector $\mathbf{s}_{k}$ which consists of $N_{t_{k}} M$ coded bits. The $i$-th symbol of $\mathbf{s}_{k}$ is $s_{i}=\left\{b_{i, 1}, b_{i, 2}, \ldots, b_{i, M}\right\}$ where $b_{i, l} \in\{ \pm 1\}$ denotes the $l$-th bit of $s_{i}$. Each user's message is coded separately. It is possible to assume that the coded bits of each symbol in $\mathbf{s}_{k}$ are independent due to the bit interleaver.

The IVLDD receiver consists of a VLD detector and a bank of soft-input soft-output (SISO) decoders for the corresponding users. The SISO decoders generate a posteriori probabilities (APPs) for each user's encoded bits, and therefore the soft estimates of the transmitted symbol. The multiuser detector jointly detects the signals transmitted by all the $K$ users.

For MU-MIMO coded systems the maximum a posteriori probability (MAP) detector is also desirable, however, its complexity grows with the 
number of transmit antennas, the constellation size and the number of active users. The MAP detector computes the a posteriori log-likelihood ratio (LLR) of a transmitted symbol for every code bit of each user as given by

$$
\begin{aligned}
\Lambda_{1}\left[b_{k, l}\right]= & \log \frac{P\left(b_{k, l}=+1 \mid \mathbf{y}\right)}{P\left(b_{k, l}=-1 \mid \mathbf{y}\right)}, \\
& k=1, \ldots, K ; \quad l=1, \ldots, M .
\end{aligned}
$$

IVLDD employs an VLD detector to compute the soft estimate $\mathbf{u}=\mathbf{T} \hat{\mathbf{z}}$ of the transmitted signal vector s after the LR-MMSE filtering, shifting and scaling operations and the transformation to the constellation domain. Then we set $\mathbf{u}=\mathbf{A} \mathbf{s}+\mathbf{v}_{e}$, where $\mathbf{A}$ is a diagonal matrix whose diagonal entries represent the users' amplitude and $\mathbf{v}_{e}$ is the effective noise, assumed Gaussian. This assumption provides an efficient way to compute the extrinsic information [85][87]. The soft output of the VLD detector for the $k$-th user is written as [36][37] [89]

$$
u_{k}=A_{k} s_{k}+v_{e_{k}},
$$

where $A_{k}$ is a scalar variable which is equal to the amplitude of the $k$-th user and $v_{e_{k}}$ is a complex Gaussian random variable with variance $\sigma_{e_{k}}^{2}$. By assuming that the symbol $u_{k}$ is statistically independent from the other symbols we have

$$
\Lambda_{1}\left[b_{k, l}\right] \approx \log \frac{P\left(b_{k, l}=+1 \mid u_{k}\right)}{P\left(b_{k, l}=-1 \mid u_{k}\right)} .
$$

Using Bayes's rule, $\Lambda_{1}\left[b_{k, l}\right]$ can be rewritten as

$$
\begin{aligned}
\Lambda_{1}\left[b_{k, l}\right] & =\log \frac{p\left(u_{k} \mid b_{k, l}=+1\right)}{p\left(u_{k} \mid b_{k, l}=-1\right)}+\log \frac{P\left(b_{k, l}=+1\right)}{P\left(b_{k, l}=-1\right)} \\
& =\lambda_{1}\left[b_{k, l}\right]+\lambda_{2}^{p}\left[b_{k, l}\right], \quad k=1, \ldots, K .
\end{aligned}
$$

where $\lambda_{2}^{p}\left[b_{k, l}\right]$ represents the a priori information for the coded bit $b_{k, l}$, which is obtained by the SISO decoder in the previous iteration. For the first iteration we assume $\lambda_{2}^{p}\left[b_{k, l}\right]=0$ for all users. The term $\lambda_{1}\left[b_{k, l}\right]$ represents the coded bit extrinsic LLR for the $k$-th user which is obtained as

$$
\lambda_{1}\left[b_{k, l}\right]=\log \frac{\sum_{a_{c} \in \mathcal{A}_{k, l}^{+}} p\left(u_{k} \mid s_{k}=a_{c}\right) P\left(s_{k}=a_{c} \mid b_{k, l}=+1\right)}{\sum_{a_{c} \in \mathcal{A}_{k, l}^{-}} p\left(u_{k} \mid s_{k}=a_{c}\right) P\left(s_{k}=a_{c} \mid b_{k, l}=-1\right)},
$$

where $\mathcal{A}_{k, l}^{ \pm}$denotes the sub sets of $\mathcal{A}$ whose $l$-th bit of $s_{k}$ is \pm 1 . Since

$$
p\left(u_{k} \mid s_{k}\right)=\frac{1}{\pi \sigma_{e_{k}}^{2}} \exp \left(\frac{-\left|u_{k}-A_{k} s_{k}\right|^{2}}{\sigma_{e_{k}}^{2}}\right),
$$

where $\sigma_{e_{k}}^{2}$ represents the variance of the effective noise of $u_{k}$. We can rewrite (413) as 


$$
\lambda_{1}\left[b_{k, l}\right]=\log \frac{\sum_{a_{c} \in \mathcal{A}_{k, l}^{+}} \exp \left(\frac{-\left|u_{k}-A_{k} a_{c}\right|^{2}}{\sigma_{e_{k}}^{2}}\right) \prod_{j \neq l} P\left(b_{k, j}\right)}{\sum_{a_{c} \in \mathcal{A}_{k, l}^{-}} \exp \left(\frac{-\left|u_{k}-A_{k} a_{c}\right|^{2}}{\sigma_{e_{k}}^{2}}\right) \prod_{j \neq l} P\left(b_{k, j}\right)},
$$

where $P\left(b_{k, j}\right)$ is a priori probability of a bit $b_{k, j}$ obtained its a priori LLR as

$$
P\left(b_{k, j}\right)=\frac{1}{2}\left[1+b_{k, j} \tanh \left(\frac{1}{2} \lambda_{2}^{p}\left[b_{k, j}\right]\right)\right] .
$$

The $\lambda_{1}\left[b_{k, l}\right]$ is de-interleaved and fed to the corresponding SISO decoder of the $k$-th user as the a priori information. The SISO decoders calculate the a posteriori LLR of each code bit by using the trellis diagram which yields

$$
\Lambda_{2}\left[b_{k, l}^{\prime}\right]=\lambda_{2}\left[b_{k, l}^{\prime}\right]+\lambda_{1}^{p}\left[b_{k, l}^{\prime}\right]
$$

The output of the SISO decoder is obtained by the a priori information $\lambda_{1}^{p}\left[b_{k, l}^{\prime}\right]$ and the extrinsic information provided by the decoder. The a posteriori LLR of every information bit is also collected by the SISO decoder which is used to make the decision of the message bit after the last iteration. The extrinsic information obtained by each SISO decoder is fed back to the detector as a priori information of all users. At the first iteration, $\lambda_{1}$ and $\lambda_{2}^{p}$ are statistically independent and as the iterations are performed they become more correlated until the improvement through iterations diminishes. The VLD is used in the first iteration to calculate the extrinsic information and then feeds it to the SISO decoders for all the users. The soft estimates $u_{k}$ are used to calculate the LLRs of their constituent bits.

Since

$$
A_{k}=\mathbb{E}\left[s_{k}^{*} u_{k}\right]
$$

and

$$
\sigma_{e_{k}}^{2}=\mathbb{E}\left[\left|u_{k}-A_{k} s_{k}\right|^{2}\right]
$$

The estimates $\hat{A}_{k}$ and $\hat{\sigma}_{e_{k}}$ required in (4-15) can be obtained by time averages of the corresponding samples over the transmitted packet which contain pilots. After the pilots, the detected symbols in previous time instants are used.

After the first iteration, the soft cancellation MMSE performs PIC by subtracting the soft replica of interference components from the received vector as

$$
\check{\mathbf{y}}=\mathbf{y}-\mathbf{H} \check{\mathbf{u}},
$$

where $\check{\mathbf{u}}=\left[u_{1}, u_{2}, \ldots, u_{k-1}, 0, u_{k+1}, \ldots, u_{K}\right]$ and a linear MMSE filter is employed to reduce the remaining interference as

$$
\mathbf{w}_{k}=\arg \min _{\mathbf{w}_{k}} \mathbb{E}\left[\left|s_{k}-\mathbf{w}_{k}^{\mathcal{H}} \check{\mathbf{y}}\right|^{2}\right]
$$

where the soft output of the filter is also Gaussian. 


\section{4 \\ Numerical Results}

In this section, we evaluate the performance of the proposed IVLDD scheme for MU-MIMO scenario. We employ a convolutional code with $R_{c}=0.5$. For each user, a packet with 998 message bits are encoded with $g=(7,5)_{\text {oct }}$ and 2000 coded bits are interleaved. These bits are modulated to 1000 QPSK symbols. We compared the IVLDD with other detectors using the same IDD receiver scheme. The two scenarios for the channels, associated with each active user, presented in Section 3.6 are used here. For the first scenario (scenario A) we consider a Gassian channel. For the second scenario (scenario B) we consider a more realistic channel with path loss, shadowing and correlation between receive and transmit antennas. The column norm based cancellation is used in the first branch of MB-LR-SIC and MB-SIC. The SNR per transmitted information bit is defined as

$$
S N R=10 \log _{10} \frac{N_{t} \sigma_{s}^{2}}{R_{c} M \sigma_{n}^{2}},
$$

where $\sigma_{s}^{2}$ is the common variance of the received symbols, $\sigma_{n}^{2}$ is the noise variance and $R_{c} M$ is the number of information bits per transmitted symbol.

In Fig. 4.3 and Fig. 4.4 we evaluate the performance of the IVLDD scheme with $L_{\text {min }}=2, c_{1}=5$ and QPSK modulation. We consider $K=5$ users with $N_{t_{i}}=2$ antennas per user, $N_{r}=10$ antennas at the BS and perfect channel estimation. The performance of different detectors with an interference free scenario, i.e., Single User with $N_{t}=1$ is compared. In Fig. 4.3, we consider scenario A for the users' channel. We can see from the plot that the performance of the iterative scheme with VLD and MB-LR-SIC schemes, after three iterations, remains close to the interference-free single-user performance. In Fig. 4.4, we show the performance of different detectors assuming perfect channel estimation with realistic conditions, scenario B, with $L_{p}=0.7, \mu=3 \mathrm{~dB}$ and $\tau=2$. The curves in Fig. 4.4 illustrate that despite the loss of performance in all detectors due to the propagation and correlation effects, the performance of the IVLDD scheme is close to the single-user scenario which is free from interference. The proposed IVLDD and iterative MB-LR-SIC schemes needs only three turbo iterations to obtain a very good BER performance due to the fact that the VLD and MB-LR-SIC receivers are able to provide a reliable estimate of the transmitted information bits since the first iteration. It is noteworthy that the IVLDD has a much lower computational cost than the iterative MB-LR-SIC scheme. 


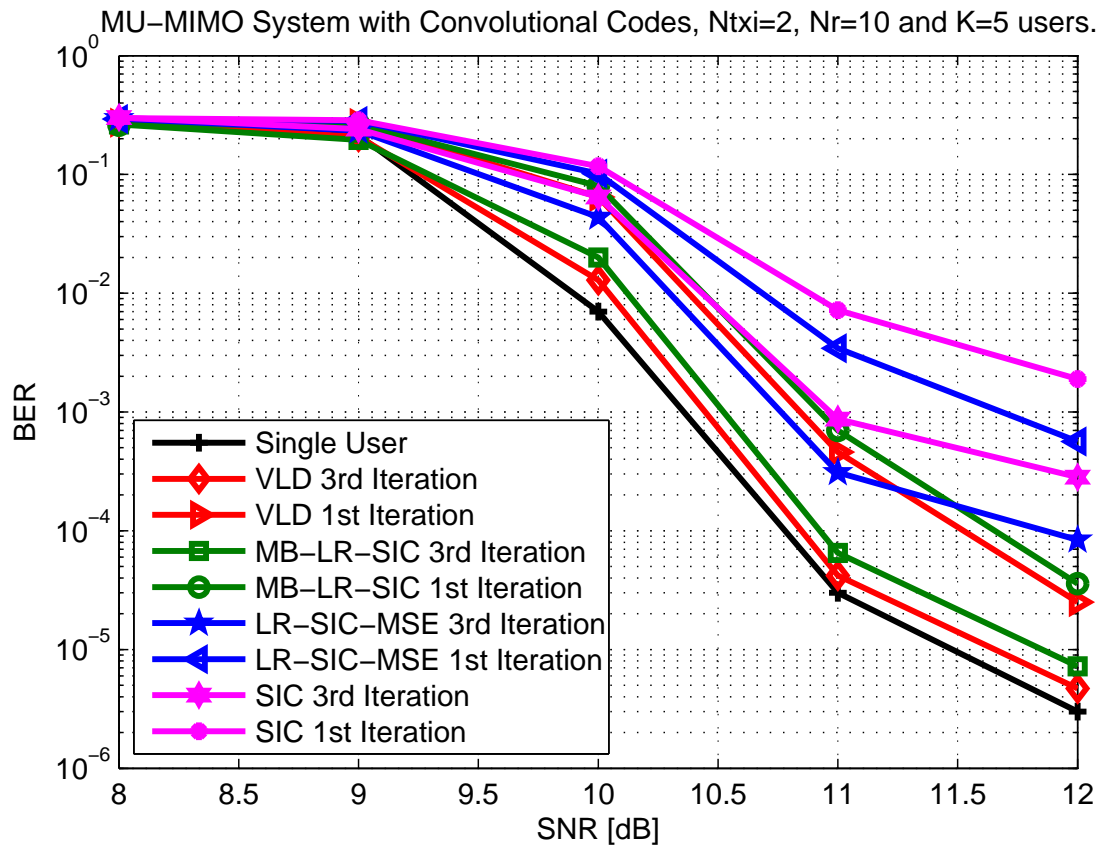

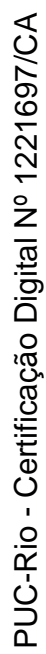

Figure 4.3: BER vs SNR performance for MU-MIMO with IDD receiver, QPSK modulation and convolutional codes with $R_{c}=0.5$ in scenario A.

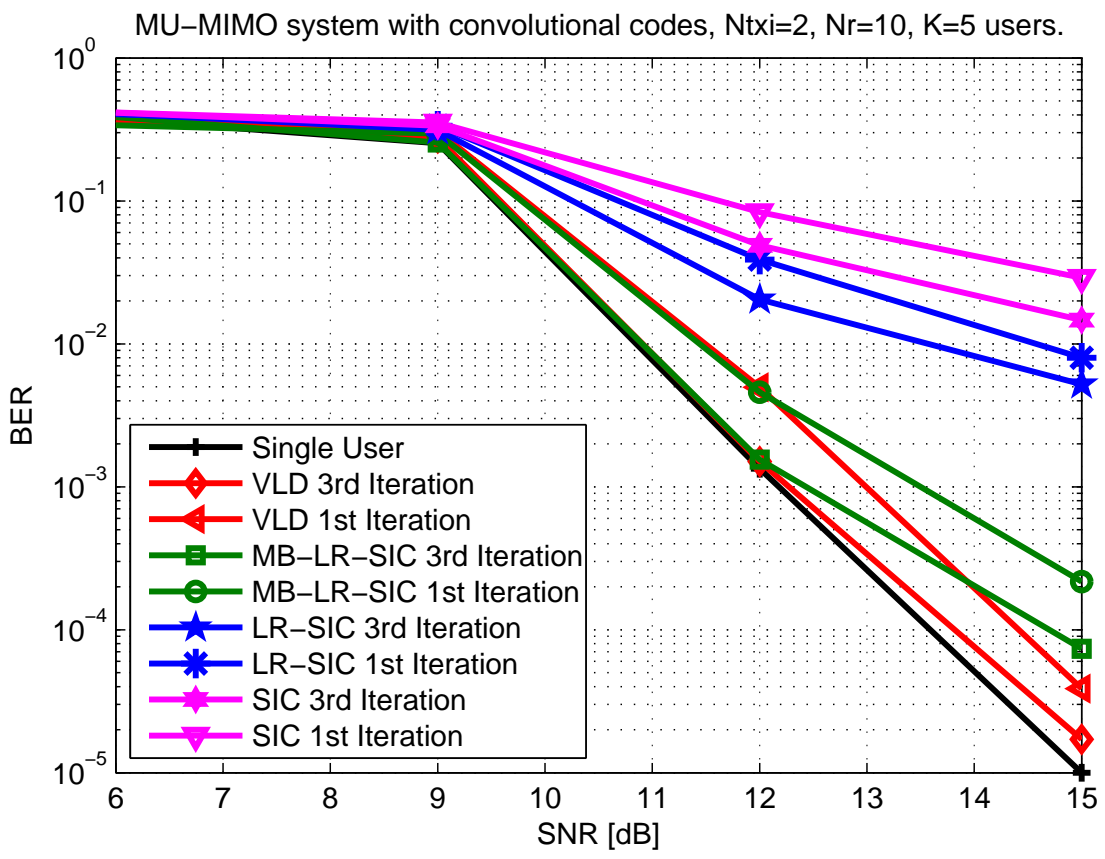

Figure 4.4: BER vs SNR performance for MU-MIMO with IDD receiver, QPSK modulation and convolutional codes with $R_{c}=0.5$ in scenario B $\left(L_{p}=0.7, \mu=3 \mathrm{~dB}, \rho_{t_{x}}=\rho_{r_{x}}=0.7\right)$. 


\section{Decoupled Signal Detection for the Uplink of Massive MIMO in Heterogeneous Cellular Networks}

\section{1 Overview}

Massive multiple-input multiple-output (MIMO) systems are strong candidates for future fifth generation $(5 \mathrm{G})$ heterogeneous cellular networks. For $5 \mathrm{G}$, a network densification with a high number of different classes of users and data service requirements is expected. Such a large number of connected devices needs to be separated in order to allow the detection of the transmitted signals according to different data requirements.

In this chapter, an algorithm to separate the received signal vector at the BS into independent signals for each user class is proposed. The proposed decoupled signal detection (DSD), allows the possibility of using more sophisticated detection schemes, as those proposed in Chapter 4, that for its high computational complexity are not recommended for massive MIMO. A signal model for massive MIMO systems with centralized and distributed antennas in heterogeneous cellular networks is also developed in this chapter. A sum rate analysis, a computational cost study and a BER performance evaluation of the proposed DSD algorithm is presented and compared with existing detection schemes in a realistic scenario with distributed antennas.

The remainder of this chapter is organized as follows. In Section 5.2 the proposed massive MIMO signal model is presented. The proposed DSD scheme is presented in Section 5.3. The sum rate analysis for the DSD scheme is described in Section 5.4. Finally, Section 5.5 presents simulations results.

\section{2}

\section{Proposed Massive MIMO Signal Model}

In this section, a signal model for heterogeneous networks with different classes of users and an arbitrary configuration of CAS and DAS is presented. We consider the uplink channel scenario of a massive MIMO system with $N$ different classes of active users transmitting simultaneously signals to one base station (BS) equipped with $D$ remote antenna arrays distributed around the cell and $N_{B}$ receive antennas at the BS. Each remote array of antennas has $Q$ antennas linked to the BS via wired links. Therefore, the total number of receive antennas is $N_{r}=N_{B}+D Q$. The choice of $N_{B}, D$ and $Q$ is made based 


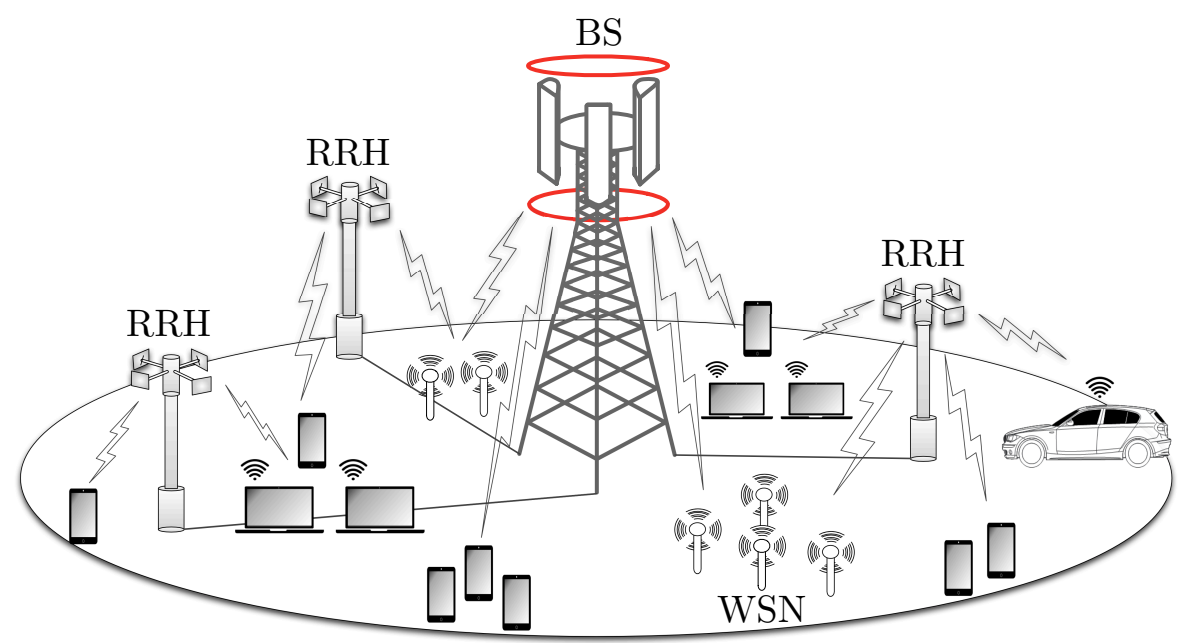

Figure 5.1: Heterogeneous Wireless Network.

on the features of the network and type of application scenario. For example, suppose that we have a city with a high density of users or devices in the centre of a cell and sparsely distributed users or devices in the remaining part of the cell. In this case, we could use a number of centralized antennas to deal with the high density of users and distributed antennas to serve the remaining devices. The cardinality of the $n$-th user class $\left|C_{n}\right|$, represents the number of users of the class $n$. The total number of active users is given by $K=\sum_{n=1}^{N}\left|C_{n}\right|$. The $k$-th user in the $n$-th user class transmits data divided into $N_{t_{k, n}}$ sub-streams through $N_{t_{k, n}}$ antennas, where $N_{r} \geqslant N_{t}=\sum_{n=1}^{N} \sum_{k=1}^{\left|C_{n}\right|} N_{t_{k, n}}$ and $N_{t}$ is the total number of transmit antennas. The received signal vector at the BS from all active users in all user classes is given by

$$
\mathbf{y}=\sum_{n=1}^{N} \sum_{k=1}^{\left|C_{n}\right|} \boldsymbol{\Upsilon}_{k, n} \overline{\mathbf{H}}_{k, n} \mathbf{s}_{k, n}+\mathbf{n}
$$

where $\mathbf{s}_{k, n}$ is the $N_{t_{k, n}} \times 1$ transmitted signal vector, by the $k$-th user of the $n$-th user class, at one time slot taken from a complex constellation, denoted by $\mathcal{A}=\left\{a_{1}, a_{2}, \ldots, a_{O}\right\}$. Each symbol has $M$ bits and $O=2^{M}$. The vector $\mathbf{n}$ is an $N_{r} \times 1$ zero mean complex circular symmetric Gaussian noise vector with covariance matrix $\mathbf{K}_{\mathbf{n}}=\mathbb{E}\left[\mathbf{n n}^{\mathcal{H}}\right]=\sigma_{n}^{2} \mathbf{I}$. Moreover, $\overline{\mathbf{H}}_{k, n}$ is the $N_{r} \times N_{t_{k, n}}$ channel matrix of the $k$-th user in the class $n$ with elements $\bar{h}_{i, j}^{(k, n)}$ corresponding to the complex channel gain from the $j$-th transmit antenna of the $k$-th user to the $i$-th receive antenna. For the antenna elements located at the $\mathrm{BS}$ and at each remote radio head, the $D+1$ sub-matrices of $\overline{\mathbf{H}}_{k, n}=\left[\left(\overline{\mathbf{H}}_{k, n}^{(1)}\right)^{T},\left(\overline{\mathbf{H}}_{k, n}^{(2)}\right)^{T}, \ldots,\left(\overline{\mathbf{H}}_{k, n}^{(D+1)}\right)^{T}\right]^{T}$ can be modeled using the Kronecker channel model, expressed by 


$$
\overline{\mathbf{H}}_{k, n}^{(j)}=\left(\mathbf{R}_{r}^{(j)}\right)^{1 / 2} \mathbf{G}_{k, n}^{(j)}\left(\mathbf{R}_{t_{k, n}}\right)^{1 / 2}
$$

where $\mathbf{G}_{k, n}^{(j)}$ has complex channel gains between the $k$-th user and the $j$-th radio head, obtained from an independent and identically distributed random fading model whose coefficients are complex Gaussian random variables with zero mean and unit variance. $\mathbf{R}_{r}^{(j)}$ and $\mathbf{R}_{t_{k, n}}$ denote the receive correlation matrix of the $j$-th radio head and the transmit correlation matrix, respectively. The components of the correlation matrices $\mathbf{R}_{r}^{(j)}$ and $\mathbf{R}_{t_{k, n}}$ are of the form:

$$
\mathbf{R}=\left[\begin{array}{ccccc}
1 & \rho & \rho^{4} & \ldots & \rho^{\left(N_{a}-1\right)^{2}} \\
\rho & 1 & \rho & \ldots & \vdots \\
\rho^{4} & \rho & 1 & \vdots & \rho^{4} \\
\vdots & \vdots & \vdots & \vdots & \vdots \\
\rho^{\left(N_{a}-1\right)^{2}} & \ldots & \rho^{4} & \rho & 1
\end{array}\right]
$$

where $N_{a}$ is the number of antennas and $\rho$ is the correlation coefficient of neighboring antennas $\left(\rho=\rho_{t_{x}}\right.$ for the transmit antennas and $\rho=\rho_{r_{x}}$ for the receive antennas). Note that $\rho=0$ represents an uncorrelated scenario and $\rho=1$ implies a fully correlated scenario. The $N_{r} \times N_{r}$ diagonal matrix $\Upsilon_{k, n}$ represents the large-scale propagation effects for the user $k$ of the user class $n$, such as path loss and shadowing, given by

$$
\boldsymbol{\Upsilon}_{k, n}=\operatorname{diag}(\underbrace{\gamma_{k, n}^{1} \ldots \gamma_{k, n}^{1}}_{N_{B}} \underbrace{\gamma_{k, n}^{2} \ldots \gamma_{k, n}^{2}}_{Q} \cdots \underbrace{\gamma_{k, n}^{D+1} \ldots \gamma_{k, n}^{D+1}}_{Q}),
$$

where the parameters $\gamma_{k, n}^{j}$ denote the large-scale propagation effects from the $k$-th user to the $j$-th radio head described by

$$
\gamma_{k, n}^{j}=\alpha_{k, n}^{j} \beta_{k, n}^{j}, \quad j=1, \ldots, D+1 .
$$

Here $\alpha_{k, n}^{j}$ is the distance based path-loss between each user and the radio heads which is calculated by

$$
\alpha_{k, n}^{j}=\sqrt{\frac{L_{k, n}^{j}}{\left(d_{k, n}^{j}\right)^{\tau}}}
$$

where $L_{k, n}^{j}$ is the power path loss of the link associated with the user and the $j$-th radio head, $d_{k, n}^{j}$ is the relative distance between this user and the $j$-th radio head, $\tau$ is the path loss exponent chosen between 2 and 4 depending on the environment. The log normal random variable $\beta_{k, n}^{j}$ which represents the shadowing between user $k$ and the receiver is given by

$$
\beta_{k, n}^{j}=10^{\frac{\mu_{k, s} \vartheta_{k, s}^{j}}{10}},
$$


where $\mu_{k, s}$ is the shadowing spread in $\mathrm{dB}$ and $\vartheta_{k, s}^{j}$ corresponds to a realvalued Gaussian random variable with zero mean and unit variance. Since the $N_{r} \times N_{t_{k, n}}$ composite channel matrix includes large-scale and small-scale fading effects it can be denoted as $\mathbf{H}_{k, n}=\boldsymbol{\Upsilon}_{k, n} \overline{\mathbf{H}}_{k, n}$, and the expression in (5-1) can be written as

$$
\mathbf{y}=\sum_{n=1}^{N} \mathbf{H}_{n} \mathbf{s}_{n}+\mathbf{n},
$$

where $\mathbf{H}_{n}=\left[\begin{array}{llll}\mathbf{H}_{1, n} & \mathbf{H}_{2, n} \ldots \mathbf{H}_{\left|C_{n}\right|, n}\end{array}\right]$ and $\mathbf{s}_{n}=\left[\begin{array}{lll}\mathbf{s}_{1, n}^{T} & \mathbf{s}_{2, n}^{T} \ldots \mathbf{s}_{\left|C_{n}\right|, n}^{T}\end{array}\right]^{T}$ represent the channel matrix and the transmitted symbol vector of all users in the class $n$, respectively. The received signal vector can be expressed more conveniently as

$$
\mathbf{y}=\mathbf{H} \mathbf{s}+\mathbf{n}
$$

where $\mathbf{H}=\left[\begin{array}{llll}\mathbf{H}_{1} & \mathbf{H}_{2} \ldots \mathbf{H}_{N}\end{array}\right]$ and $\mathbf{s}=\left[\begin{array}{lll}\mathbf{s}_{1}^{T} & \mathbf{s}_{2}^{T} \ldots \mathbf{s}_{N}^{T}\end{array}\right]^{T}$. The symbol vector $\mathbf{s}$ of all $N$ user classes has zero mean and a covariance matrix $\mathbf{K}_{\mathbf{s}}=\mathbb{E}\left[\mathbf{s s}^{\mathcal{H}}\right]=\operatorname{diag}(\mathbf{p})$, where the elements of the vector $\mathbf{p}$ are the signal power of each transmit antenna. To maintain a notational simplicity in the subsequent analysis, we assume that all antenna elements at the users transmit with the same average transmitted power $\sigma_{s}^{2}$, i.e., $\mathbf{K}_{\mathbf{s}}=\sigma_{s}^{2} \mathbf{I}$. We assume that the channel matrix $\mathbf{H}$ was previously estimated at the BS. From (5-9) we can see that the signals arrive coupled at the BS. If we want to use different detection procedures for each user class according to its data requirements, we have to separate the received signal vector $\mathbf{y}$ into independent received signals for each user class. For the system model presented in this thesis, when the number of remote radio heads is set to zero, i.e., $D=0$, the DAS architecture is reduced to the CAS scheme with $N_{r}=N_{B}$.

\section{3}

\section{Decoupled Signal Detection}

As presented in Section 5.2, in heterogeneous networks different classes of users send parallel data streams, through the massive MIMO channel operating with distributed antennas, which arrive superposed at the BS. In this $5 \mathrm{G}$ context, we need to separate the data streams for each category of users efficiently. In this section, we describe the proposed decoupled signal detection (DSD) which allows us to separate the received signal of the $n$ th user class from the others. To this end, we consider that the process of authentication, identification and channel estimation was already made, i.e, the BS is able to identify the users by classes according to their data requirements. 
Similar approaches to the proposed DSD algorithm have been proposed for the downlink, such as block diagonalization (BD) based techniques [90]- [93]. However, unlike prior work in which downlink BD is used, for the proposed DSD scheme it is not necessary to use any precoding at the transmit side. The receiver only needs to know the channels between users and receive antennas. Moreover, the concept of separating the users with respect to the classes in heterogeneous networks according to its requirements is a new approach. The first steps to construct the concept proposed in this thesis were presented in [66] [94]. The received signal vector (5-8) can be written as:

$$
\mathbf{y}=\mathbf{H}_{n} \mathbf{s}_{n}+\sum_{m=1, m \neq n}^{N} \mathbf{H}_{m} \mathbf{s}_{m}+\mathbf{n},
$$

where $\mathbf{H}_{n}$ and $\mathbf{s}_{n}$ are the channel matrix and the transmitted symbol vector for the $n$-th user class, respectively. From (5-10), we can see that the $n$-th user class has inter-user class interference.

\subsection{1}

\section{Proposed Decoupling Strategy}

To remove the presence of the other classes of users in the detection procedure for the $n$-th user class, we can employ a linear operation to project the received signal vector $\mathbf{y}$ onto the subspace orthogonal or almost orthogonal to the subspace generated by the signals of the interfering classes. In DSD, a matrix $\mathbf{A}_{n}$ is calculated employing a channel inversion method and a $\mathrm{QR}$ decomposition [95] [96], in order to decouple the $n$-th user's class received signal from other user's class signals. To compute $\mathbf{A}_{n}$, we construct the matrix $\tilde{\mathbf{H}}_{n}$ excluding the channel matrix of the $n$-th user class in the following form:

$$
\tilde{\mathbf{H}}_{n}=\left[\begin{array}{lll}
\mathbf{H}_{1} \ldots \mathbf{H}_{n-1} & \mathbf{H}_{n+1} \ldots \mathbf{H}_{N}
\end{array}\right]
$$

where $\tilde{\mathbf{H}}_{n} \in \mathbb{Q}^{N_{r} \times\left(N_{t}-N_{t_{n}}\right)}$ and $N_{t_{n}}=\sum_{k=1}^{\left|C_{n}\right|} N_{t_{k, n}}$ is the number of transmit antennas in the $n$-th user class. After that, the objective is to obtain a matrix $\mathbf{A}_{n}$ that satisfies the following condition:

$$
\mathbf{A}_{n} \tilde{\mathbf{H}}_{n}=\mathbf{0}, \quad \forall n \in(1 \ldots N)
$$

To compute $\mathbf{A}_{n}$, DSD first computes the MMSE channel inversion of the combined channel matrix $\mathbf{H}$ given by

$$
\begin{aligned}
\mathbf{H}^{\dagger} & =\mathbf{H}^{\mathcal{H}}\left(\mathbf{H H}^{\mathcal{H}}+\sigma^{2} \mathbf{I}\right)^{-1} \\
& =\left[\begin{array}{llll}
\ddot{\mathbf{H}}_{1}^{T} & \ddot{\mathbf{H}}_{2}^{T} & \ldots & \ddot{\mathbf{H}}_{N}^{T}
\end{array}\right]^{T}
\end{aligned}
$$


where $\sigma^{2}=\sigma_{n}^{2} / \sigma_{s}^{2}, \mathbf{H}^{\dagger} \in \mathbb{Q}^{N_{t} \times N_{r}}$ and $\ddot{\mathbf{H}}_{n} \in \mathbb{Q}^{N_{t_{n}} \times N_{r}}$. Then, the matrix $\ddot{\mathbf{H}}_{n}$ is approximately in the null space of $\tilde{\mathbf{H}}_{n}$, that is

$$
\ddot{\mathbf{H}}_{n} \tilde{\mathbf{H}}_{n} \approx \mathbf{0}, \quad \forall n \in(1 \ldots N) .
$$

To decouple the $n$-th user group from the other user groups we employ a QR decomposition as described by

$$
\ddot{\mathbf{H}}_{n}=\mathbf{R}_{n} \mathbf{Q}_{n}, \quad \forall n \in(1 \ldots N),
$$

where $\mathbf{R}_{n} \in \mathbb{Q}^{N_{t_{n}} \times N_{t_{n}}}$ is an upper triangular matrix and $\mathbf{Q}_{n} \in \mathbb{Q}^{N_{t_{n}} \times N_{r}}$ is a matrix with orthogonal rows and composed by approximately orthonormal basis vectors of the left null space of $\tilde{\mathbf{H}}_{n}$. Then we have

$$
\mathbf{Q}_{n} \tilde{\mathbf{H}}_{n} \approx \mathbf{0} \text {. }
$$

From (5-16), we can see that $\mathbf{Q}_{n}$ is a good approximation for $\mathbf{A}_{n}$ in (5-12). Using $\mathbf{A}_{n}=\mathbf{Q}_{n}$ as a linear combination with the received signal vector in (510), we have

$$
\begin{aligned}
\mathbf{y}_{n} & =\mathbf{A}_{n} \mathbf{y} \\
& =\mathbf{Q}_{n} \mathbf{H}_{n} \mathbf{s}_{n}+\mathbf{Q}_{n} \sum_{\substack{m=1 \\
m \neq n}}^{N} \mathbf{H}_{m} \mathbf{s}_{m}+\mathbf{Q}_{n} \mathbf{n},
\end{aligned}
$$

where $\mathbf{y}_{n} \in \mathbb{Q}^{N_{t_{n}} \times 1}$ is the equivalent received signal vector for the user class $n$ and the term $\mathbf{Q}_{n} \sum_{m .1}^{N} \mathbf{H}_{m} \mathbf{s}_{m} \approx \mathbf{0}$ represents the residual inter-user class interference. Then, we can transform the received signal vector into parallel single-user class signals as described by

$$
\mathbf{y}_{n}=\check{\mathbf{H}}_{n} \mathbf{s}_{n}+\mathbf{n}_{n}, \quad \forall n \in(1 \ldots N),
$$

where $\check{\mathbf{H}}_{n}=\mathbf{Q}_{n} \mathbf{H}_{n} \in \mathbb{Q}^{N_{t_{n}} \times N_{t_{n}}}$ is the equivalent channel matrix of the $n$-th user class after DSD and $\mathbf{n}_{n}=\mathbf{Q}_{n} \sum_{m .1}^{N} \mathbf{H}_{m} \mathbf{s}_{m}+\mathbf{Q}_{n} \mathbf{n} \in \mathbb{Q}^{N_{t_{n}} \times 1}$ is the equivalent noise vector.

Another option to compute a basis for the left null space of $\tilde{\mathbf{H}}_{n}$ is performing the SVD transformation $\tilde{\mathbf{H}}_{n}=\tilde{\mathbf{U}}_{n} \tilde{\boldsymbol{\Sigma}}_{n} \tilde{\mathbf{V}}_{n}^{\mathcal{H}}$, where $\tilde{\boldsymbol{\Sigma}}_{n} \in \mathbb{Q}^{N_{r} \times\left(N_{t}-N_{t_{n}}\right)}$ is a rectangular diagonal matrix with the singular values of $\tilde{\mathbf{H}}_{n}$ on the diagonal, $\tilde{\mathbf{U}}_{n} \in \mathbb{Q}^{N_{r} \times N_{r}}$ and $\tilde{\mathbf{V}}_{n}^{\mathcal{H}} \in \mathbb{Q}^{\left(N_{t}-N_{t_{n}}\right) \times\left(N_{t}-N_{t_{n}}\right)}$ are unitary matrices. If $r_{n}$ is the rank of $\tilde{\mathbf{H}}_{n}$, that corresponds to the number of non-zero singular values, i.e., $r_{n}=\operatorname{rank}\left(\tilde{\mathbf{H}}_{n}\right) \leq N_{t}-N_{t_{n}}$, the SVD can be expressed equivalently as:

$$
\tilde{\mathbf{H}}_{n}=\left[\begin{array}{ll}
\tilde{\mathbf{U}}_{1, n} & \tilde{\mathbf{U}}_{0, n}
\end{array}\right] \tilde{\boldsymbol{\Sigma}}_{n}\left[\begin{array}{ll}
\tilde{\mathbf{V}}_{1, n} & \tilde{\mathbf{V}}_{0, n}
\end{array}\right]^{\mathcal{H}},
$$

where $\tilde{\mathbf{U}}_{0, n} \in \mathbb{Q}^{N_{r} \times\left(N_{r}-r_{n}\right)}$ and $\tilde{\mathbf{V}}_{0, n}^{\mathcal{H}} \in \mathbb{Q}^{\left(N_{t}-N_{t_{n}}-r_{n}\right) \times\left(N_{t}-N_{t_{n}}\right)}$ form an orthogonal 
basis for the left null space and the null space of $\tilde{\mathbf{H}}_{n}$, respectively. Then, the alternative solution for (5-12) could be:

$$
\mathbf{A}_{n}=\tilde{\mathbf{U}}_{0, n}^{\mathcal{H}} .
$$

Although the matrix $\tilde{\mathbf{U}}_{0, n}^{\mathcal{H}}$ eliminates the inter-user class interference effectively, i.e., $\tilde{\mathbf{U}}_{0, n}^{\mathcal{H}} \sum_{\substack{m . n \\ m . n}}^{N} \mathbf{H}_{m} \mathbf{s}_{m}=\mathbf{0}$, the linear combination $\mathbf{y}_{n}=\tilde{\mathbf{U}}_{0, n}^{\mathcal{H}} \mathbf{y}$ presents a noise enhancement in the detection procedures which reduces the performance. In the first approach, when we use $\mathbf{y}_{n}=\mathbf{Q}_{n} \mathbf{y}$, the noise enhancement in the detection procedures is mitigated due to the fact that the equivalent noise vector has a lower dimension when compared with the other option. Thus, the noise enhancement is reduced which improves the performance, even in the presence of residual interference. In addition, the equivalent channel matrix $\check{\mathbf{H}}_{n}^{1}=\tilde{\mathbf{U}}_{0, n}^{\mathcal{H}} \mathbf{H}_{n}$ has dimensions $\left(N_{r}-r\right) \times N_{t}$ against the matrix $\check{\mathbf{H}}_{n}^{2}=\mathbf{Q}_{n} \mathbf{H}_{n}$ which has dimensions $N_{t_{n}} \times N_{t_{n}}$. For this reason the computational complexity of the detector is lower if we use the matrix $\mathbf{Q}_{n}$ to decouple the received signal vector.

The fact that we obtain a square equivalent channel matrix also allows the possibility of using lattice reduction (LR) based detectors which have a better performance for square channel matrices [69]. Further, the computational complexity to compute the channel inversion (5-13) and $N \mathrm{QR}$ decompositions (5-15) of matrices with dimensions $N_{t_{n}} \times N_{r}$ is much lower than the computational cost of computing $N$ SVD transformations (5-19) of matrices with dimensions $N_{r} \times\left(N_{t}-N_{t_{n}}\right)$. For these reasons, in this thesis we will focus on the first alternative.

As it will be presented in next section, the equivalent received signal vector in (5-18) shows that the process in (5-11)-(5-17) is an effective algorithm to separate the user classes at the BS and we can consider the data stream of the $n$-th user class as independent of the received signals of the other user classes. In practice, this allows the possibility of using different transmission and reception schemes for each user class. We can now implement the traditional detectors for each class of users separately which also allows the possibility of using more complex detection schemes due to the reduction of the dimensions of the matrices that need to be processed. The description of the proposed DSD algorithm is presented in Algorithm 4.

\section{3 .2}

\section{Detection Algorithms}

In this subsection, we examine signal detection algorithms for massive MIMO in heterogeneous networks. To detect the data stream for each class 


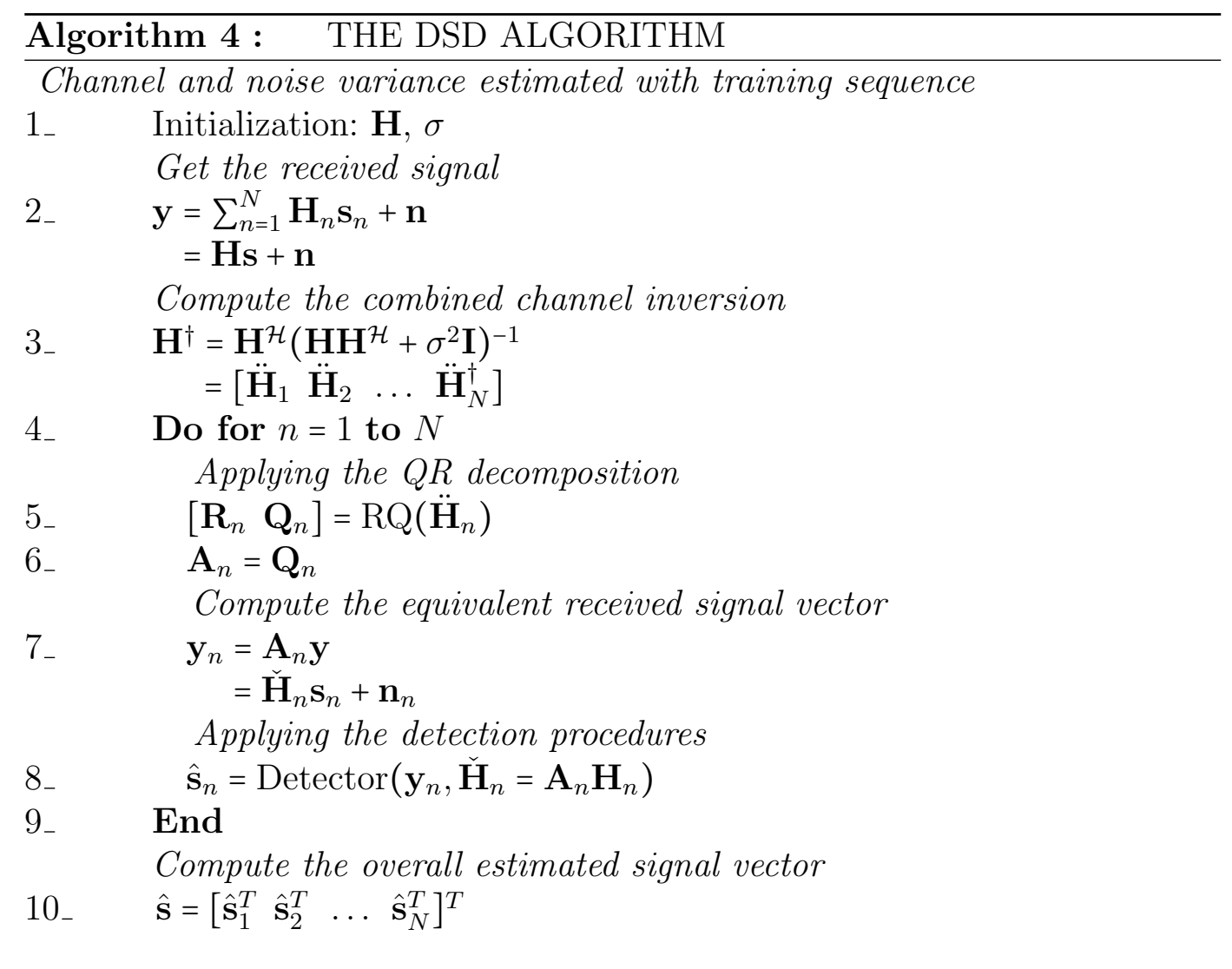

of users independently, we assume that the DSD algorithm described in Algorithm 4 was previously employed.

\section{Linear Detectors}

In linear detectors, the equivalent received signal vector for the $n$-th user class $\mathbf{y}_{n} \in \mathbb{Q}^{N_{t_{n} \times 1}}$ is processed by a linear filter to eliminate the channel effects [33]. The two linear detectors considered here are given by

$$
\mathbf{W}_{n}^{\chi}= \begin{cases}\left(\check{\mathbf{H}}_{n}^{\mathcal{H}} \check{\mathbf{H}}_{n}\right)^{-1} \check{\mathbf{H}}_{n}^{\mathcal{H}}, & \chi=\text { Zero Forcing } \\ \left(\check{\mathbf{H}}_{n}^{\mathcal{H}} \check{\mathbf{H}}_{n}+\sigma^{2} \mathbf{I}\right)^{-1} \check{\mathbf{H}}_{n}^{\mathcal{H}}, & \chi=\text { MMSE }\end{cases}
$$

where $\sigma^{2}=\sigma_{n}^{2} / \sigma_{s}^{2}$ and $\check{\mathbf{H}}_{n} \in \mathbb{Q}^{N_{t_{n}} \times N_{t_{n}}}$ is the equivalent channel matrix of the $n$-th user class. Note that for the MMSE detector, we consider the autocorrelation matrix of the equivalent noise vector as $\mathbf{K}_{\mathbf{n}_{n}} \approx \sigma_{n}^{2} \mathbf{I}$. As the residual interference is very small, an excellent performance can be obtained with this approximation. The linear hard decision of $\mathbf{s}_{n}$ is carried out as follows:

$$
\hat{\mathbf{s}}_{n}=\mathbb{C}\left(\mathbf{W}_{n}^{\chi} \mathbf{y}_{n}\right),
$$

where the function $\mathbb{C}(x)$ returns the point of the complex signal constellation closest to $x$. 


\section{Successive Interference Cancellation (SIC)}

The SIC detector for the $n$-th user class in (5-18) consists of a bank of linear detectors, each detects a selected component $s_{n, i}$ of $\mathbf{s}_{n}$. The component obtained by the first detector is used to reconstruct the corresponding signal vector which is then subtracted from the equivalent received signal to further reduce the interference in the input to the next linear receive filter. The successively canceled received data vector that follows a chosen ordering in the $i$-th stage is given by

$$
\mathbf{y}_{n, i}=\mathbf{y}_{n}-\sum_{j=1}^{i-1} \check{\mathbf{h}}_{n, j} \hat{s}_{n, j},
$$

where $\check{\mathbf{h}}_{n, j}$ correspond to the columns of the channel matrix $\check{\mathbf{H}}_{n}$ and $\hat{s}_{n, j}$ is the estimated symbol obtained at the output of the $j$-th linear detector.

\section{Multiple-Branch SIC Detection}

In the multi-branch scheme [37] for the $n$-th user class, different orderings are explored for SIC, each ordering is referred to as a branch, so that a detector with $L$ branches produces a set of $L$ estimated vectors. Each branch uses a column permutation matrix $\mathbf{P}_{n}$. The estimate of the signal vector of branch $l, \hat{\mathbf{x}}_{n}^{(l)}$, is obtained using a SIC receiver based on a new channel matrix $\check{\mathbf{H}}_{n}^{(l)}=\check{\mathbf{H}}_{n} \mathbf{P}_{n}^{(l)}$. The order of the estimated symbols is rearranged to the original order by

$$
\hat{\mathbf{s}}_{n}^{(l)}=\mathbf{P}_{n}^{(l)} \hat{\mathbf{x}}_{n}^{(l)}, \quad l=1, \ldots, L .
$$

A higher detection diversity can be obtained by selecting the most likely symbol vector based on the ML selection rule, that is

$$
\hat{\mathbf{s}}_{n}=\arg \min \left\|\mathbf{y}_{n}-\check{\mathbf{H}}_{n} \hat{\mathbf{s}}_{n}^{l}\right\|^{2}, \quad l=1, \ldots, L .
$$

Other detectors as the proposed MB-LR-SIC and VLD in chapter 4, could be used with the proposed DSD technique and this is up to the designer to choose the detector.

\section{4}

\section{Sum-Rate Analysis}

In this section, a performance analysis for the proposed DSD scheme is presented in terms of the sum rate. We consider that the channel matrix $\mathbf{H}$ was previously estimated at the BS, assume Gaussian signalling and that the received signal vector was decoupled for each user class. Considering the received signal vector as presented in (5-18), the sum rate [97] that DSD can offer is defined as 


$$
R=\sum_{n=1}^{N} \log _{2} \frac{\operatorname{det}\left(\mathbf{K}_{\mathbf{y}_{n}}\right)}{\operatorname{det}\left(\mathbf{K}_{\mathbf{n}_{n}}\right)}
$$

where $\mathbf{K}_{\mathbf{y}_{n}}$ and $\mathbf{K}_{\mathbf{n}_{n}}$ are the autocorrelation matrix of the equivalent received signal vector and the equivalent noise vector of the $n$-th user class, respectively. It is easy to show that (5-26), can be expressed as

$$
R=\sum_{n=1}^{N} \log _{2} \operatorname{det}\left(\mathbf{I}+\mathbf{B}_{n} \mathbf{B}_{n}^{\mathcal{H}}\right)
$$

where $\mathbf{B}_{n}=\mathbf{K}_{\mathbf{n}_{n}}^{-1 / 2} \check{\mathbf{H}}_{s} \mathbf{K}_{\mathbf{s}_{n}}^{1 / 2} \in \mathbb{Q}^{N_{t_{n}} \times N_{t_{n}}}$. As $\mathbf{B}_{n} \mathbf{B}_{n}^{\mathcal{H}}$ is a Hermitian symmetric positive-definite square matrix, we have

$$
\mathbf{B}_{n} \mathbf{B}_{n}^{\mathcal{H}}=\overline{\mathbf{Q}}_{n} \boldsymbol{\Lambda}_{n} \overline{\mathbf{Q}}_{n}^{\mathcal{H}}
$$

where $\overline{\mathbf{Q}}_{n}$ is a square unitary matrix, $\overline{\mathbf{Q}}_{n} \overline{\mathbf{Q}}_{n}^{\mathcal{H}}=\mathbf{I}$, and $\boldsymbol{\Lambda}_{n}$ is a diagonal matrix whose diagonal elements are the eigenvalues of the matrix $\mathbf{B}_{n} \mathbf{B}_{n}^{\mathcal{H}}$. Then, the reliable sum rate that the system can offer is

$$
R=\sum_{n=1}^{N} \sum_{i=1}^{N_{t_{n}}} \log _{2}\left(1+\lambda_{i, n}\right) .
$$

Note that the eigenvalues $\lambda_{i, n}$ in (5-29) can be obtained computing the eigenvalues of $\mathbf{B}_{n}^{\mathcal{H}} \mathbf{B}_{n}$. As mentioned before, for notational simplicity we assume that $\mathbf{K}_{\mathbf{s}_{n}}=\sigma_{s}^{2} \mathbf{I}$. When the DSD algorithm is applied, the equivalent noise vector for the $n$-th user class $\mathbf{n}_{n}=\mathbf{Q}_{n} \sum_{\substack{m .1 \\ m . n}}^{N} \mathbf{H}_{m} \mathbf{s}_{m}+\mathbf{Q}_{n} \mathbf{n} \in \mathbb{Q}^{N_{t_{n}} \times 1}$ is not white due to the residual inter-user class interference. Then its autocorrelation matrix is given by

$$
\mathbf{K}_{\mathbf{n}_{n}}=\mathbb{E}\left[\mathbf{n}_{n} \mathbf{n}_{n}^{\mathcal{H}}\right]=\sigma_{s}^{2} \mathbf{Q}_{n}\left(\sum_{\substack{m=1 \\ m \neq n}}^{N} \mathbf{H}_{m} \mathbf{H}_{m}^{\mathcal{H}}\right) \mathbf{Q}_{n}^{\mathcal{H}}+\sigma_{n}^{2} \mathbf{I} .
$$

Finally, the eigenvalues $\lambda_{i, n}$ in (5-29) are obtained from the eigenvalues of matrix $\mathbf{B}_{n}^{\mathcal{H}} \mathbf{B}_{n}$ given by

$$
\mathbf{B}_{n}^{\mathcal{H}} \mathbf{B}_{n}=\frac{\sigma_{s}^{2}}{\sigma_{n}^{2}} \check{\mathbf{H}}_{n}^{\mathcal{H}}\left[\frac{\sigma_{s}^{2}}{\sigma_{n}^{2}} \mathbf{Q}_{n}\left(\sum_{\substack{m=1 \\ m \neq n}}^{N} \mathbf{H}_{m} \mathbf{H}_{m}^{\mathcal{H}}\right) \mathbf{Q}_{n}^{\mathcal{H}}+\mathbf{I}\right]^{-1} \check{\mathbf{H}}_{n} .
$$

To compute the sum rate for the received signal vector in (5-9) when the detection is perform for all user classes together, we suppress the index $n$ from the above analysis and considering that $\mathbf{K}_{\mathbf{s}}=\sigma_{s}^{2} \mathbf{I}$ and $\mathbf{K}_{\mathbf{n}}=\sigma_{n}^{2} \mathbf{I}$, we get the well-known expression:

$$
\bar{R}=\sum_{i=1}^{N_{t}} \log _{2}\left(1+\lambda_{i}\right)
$$

where the values $\lambda_{i}$ are the eigenvalues of the matrix $\mathbf{B}^{\mathcal{H}} \mathbf{B}=\frac{\sigma_{s}^{2}}{\sigma_{n}^{2}} \mathbf{H}^{\mathcal{H}} \mathbf{H}$ [97]. In Appendix C, we show that, as well as the sum rate in (5-32) when all user classes are detected together, the sum rate in (5-29) for the proposed DSD algorithm is independent of the detection procedure. However, the lower bound 
on the achievable uplink sum rate obtained by using linear detectors is different for each detector [98]. In order to analyze the behavior of the lower bound on the sum rate for the proposed DSD scheme we consider that a linear detector according to (5-21) is applied to the equivalent received signal vector (5-18) to detect the transmitted symbol vector of the user class $n$, then we have

$$
\tilde{\mathbf{y}}_{n}=\mathbf{W}_{n} \mathbf{y}_{n}=\mathbf{W}_{n} \check{\mathbf{H}}_{n} \mathbf{s}_{n}+\mathbf{W}_{n} \mathbf{n}_{n}
$$

Taking the $k$-th element of $\tilde{\mathbf{y}}_{n}$ we have

$$
\tilde{y}_{k, n}=\mathbf{w}_{k, n} \check{\mathbf{h}}_{k, n} s_{k, n}+\sum_{\substack{j=1 \\ j \neq k}}^{\left|C_{n}\right|} \mathbf{w}_{k, n} \check{\mathbf{h}}_{j, n} s_{j, n}+\mathbf{w}_{k, n} \mathbf{n}_{n}
$$

where $\mathbf{w}_{k, n}$ is the $k$-th row of $\mathbf{W}_{n}$. Modeling the noise interference, the interuser class interference and the inter-user interference in the user class $n$ in (534) as additive Gaussian noise independent of $s_{k, n}$, considering (5-30) and that the channel is ergodic so that each codeword spans over a large number of realizations, we obtain the lower bound on the achievable rate for the DSD algorithm with linear detectors as

$$
\begin{aligned}
R_{k, n}= & \mathbb{E}\left[\log _{2}(1+\ldots\right. \\
& \left.\left.\frac{\sigma_{s}^{2}\left|\mathbf{w}_{k, n} \check{\mathbf{h}}_{k, n}\right|^{2}}{\sigma_{s}^{2} \sum_{\substack{j=1 \\
j \neq k}}^{\left|C_{n}\right|}\left|\mathbf{w}_{k, n} \check{\mathbf{h}}_{j, n}\right|^{2}+\left|\mathbf{w}_{k, n}\left[\sigma_{s}^{2} \mathbf{Q}_{n}\left(\sum_{\substack{m=1 \\
m \neq n}}^{N} \mathbf{H}_{m} \mathbf{H}_{m}^{\mathcal{H}}\right) \mathbf{Q}_{n}^{\mathcal{H}}+\sigma_{n}^{2} \mathbf{I}\right] \mathbf{w}_{k, n}^{\mathcal{H}}\right|}\right)\right] .
\end{aligned}
$$

Then, the lower bound on the achievable rate for the entire system is given by

$$
R=\sum_{n=1}^{N} \sum_{k=1}^{\left|C_{n}\right|} R_{k, n}
$$

For the SIC receiver, each stream is filtered by a linear detector and then, its contribution is subtracted from the received signal to improve the subsequent detection. For each layer the linear filter is recalculated. The performance of SIC detectors can be improved if we choose the cancellation order as a function of the SINR at the output of the linear detector in each layer. The lower bound for the sum rate of the proposed DSD algorithm when a SIC detector is used for each user class, could be calculated updating the expression (5-35) in each layer, i.e., the values of $\mathbf{w}_{k, n}$ are recalculated for each detected stream. 


\section{5}

\section{Numerical Results}

In this section, we evaluate the performance of the proposed DSD algorithm with different detectors in terms of the sum rate and the BER via simulations. Moreover, the computational complexity of the proposed and existing algorithms is also evaluated.

\subsection{1}

\section{Sum Rate}

To evaluate the analytic results obtained in Section 5.4, the sum rate and the lower bounds for the proposed DSD algorithm with different detection schemes will be evaluated considering CAS and DAS configuration assuming perfect CSI. For the CAS configuration, we employ $L_{k}=0.7, \tau=2$, the distance $d_{k}$ to the BS is obtained from a uniform discrete random variable distributed between 0.1 and 0.99 , the shadowing spread is $\sigma_{k}=3 \mathrm{~dB}$ and the transmit and receive correlation coefficients are $\rho_{r x}=0.2$ and $\rho_{t x}=0.4$ (when $N_{t_{k, n}}>1$ ), respectively. For DAS configurations, we consider a densely populated cell, where a fraction of the active users are in the centre of the cell and the remaining users are in other locations of the cell. We explore different particular values for the fraction of users in the centre and around the cell. Based on that, we choose specific values for $N_{B}, D$ and $Q$. For the DAS configuration, we also consider $L_{k, j}$ taken from a uniform random variable distributed between 0.7 and $1, \tau=2$, the distance $d_{k, j}$ for each link to an antenna is obtained from a uniform discrete random variable distributed between 0.1 and 0.5, the shadowing spread is $\sigma_{k, j}=3 \mathrm{~dB}$ and the transmit and receive correlation coefficients are $\rho_{r x}=0.2$ and $\rho_{t x}=0.4$, respectively.

In Fig. 5.2 we evaluate the sum rate in two different scenarios for the users requirements. In both cases, we fix the $\mathrm{SNR}=10 \mathrm{~dB}$ and increase the number of receive antennas. For the DAS configuration, we consider $N_{B}=1 / 2 N_{r}$ antennas at the BS. We also consider $D=4$ arrays of antennas distributed around the cell, each equipped with $Q=1 / 8 N_{r}$ antennas. For Fig. 5.2 (a) we consider $N=4$ classes of users with $\left|C_{n}\right|=8$ users each and $N_{t_{k, n}}=1$ antennas per user. We can see that the sum rate of the proposed DSD algorithm is close to the sum rate of the traditional MIMO system and with a low computational complexity on the detection procedures as will be shown in the next subsection. For Fig. 5.2 (b), we consider 16 active users in the system and that we need to detect each user independently, i.e., $N=16$ classes of users with $\left|C_{n}\right|=1$ users at each class and $N_{t_{k, n}}=2$ antennas per user. Under these conditions, the sum rate of the proposed scheme reaches the sum rate of the traditional 


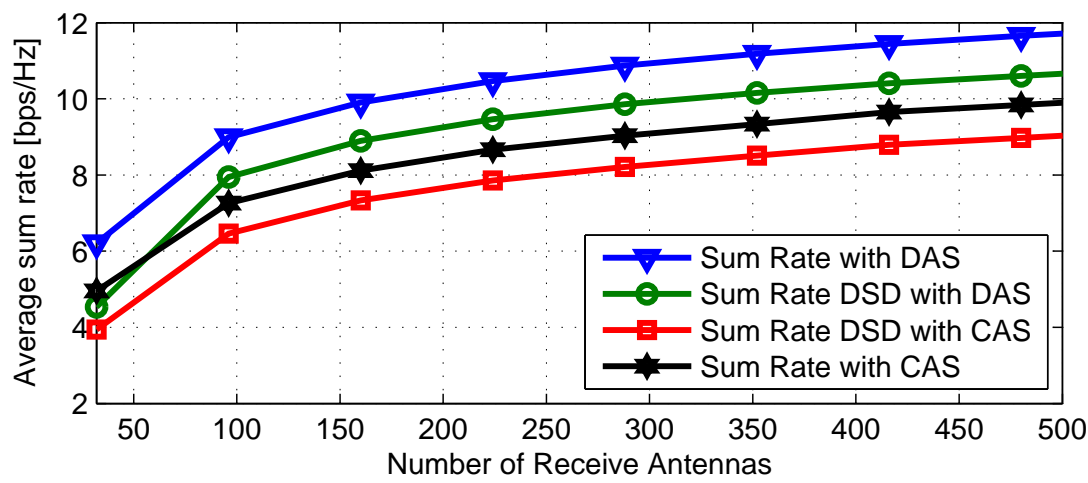

(a)

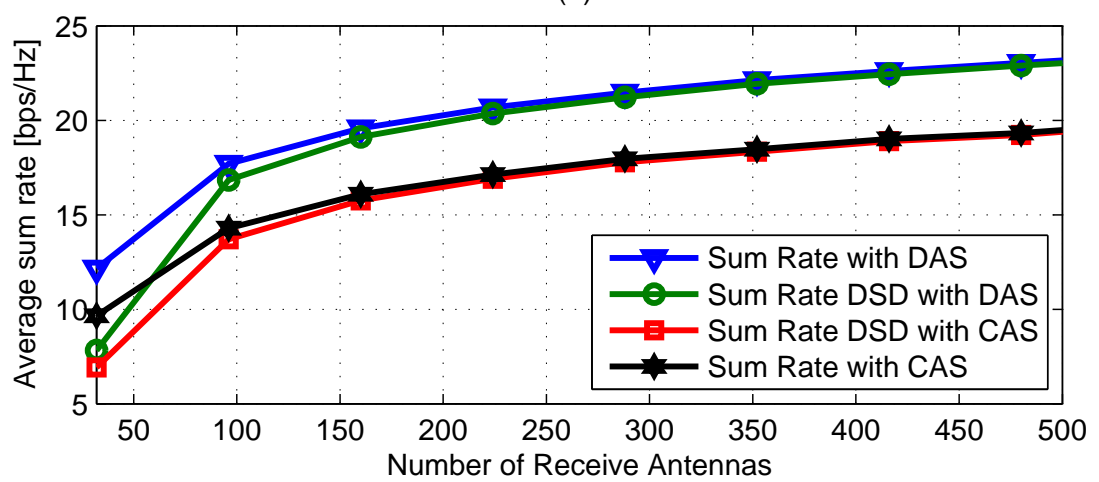

(b)

Figure 5.2: (a) Sum Rate vs Number of Receive antennas. $N=4,\left|C_{n}\right|=8$ users per class, $N_{t_{k, n}}=1$ antennas per user. (b) Sum Rate vs Number of Receive antennas. $N=16,\left|C_{n}\right|=1$ users per class, $N_{t_{k, n}}=2$ antennas per user.

MIMO system, especially for a large number of receive antennas. As expected, from the plot in Fig. 5.2, we can see that the sum rate for DAS is higher than that of the CAS configuration.

In Fig. 5.3, we compare the lower bound on the sum rate for different detectors such as, ZF, MMSE and SIC. We consider the DAS configuration under the same conditions as in the previous experiment. For Fig. 5.3 (a) we consider $N=3$ classes of users with $\left|C_{n}\right|=10$ users at each class and $N_{t_{k, n}}=1$ antennas per user. We can see from the plot, that similarly to the traditional MIMO systems, the lower bound on the sum rate for ZF and MMSE achieves the sum rate when $N_{r}$ grows. For Fig. 5.3 (b) we consider $N=2$ classes of users with $\left|C_{n}\right|=16$ users at each class and $N_{t_{k, n}}=1$ antennas per user. We can see that the SIC-MMSE achieves the sum rate and it could be considered optimal in terms of sum rate.

In Fig. 5.4, we compare the lower bound sum rate versus SNR. We consider $N=8$ classes of users with $\left|C_{n}\right|=1$ user in each class and $N_{t_{k, n}}=8$ antennas per user transmitting with high correlation between antennas $\rho_{t x}=$ 0.85. We consider the DAS configuration with $N_{B}=96, D=4$ and $Q=8$. We 


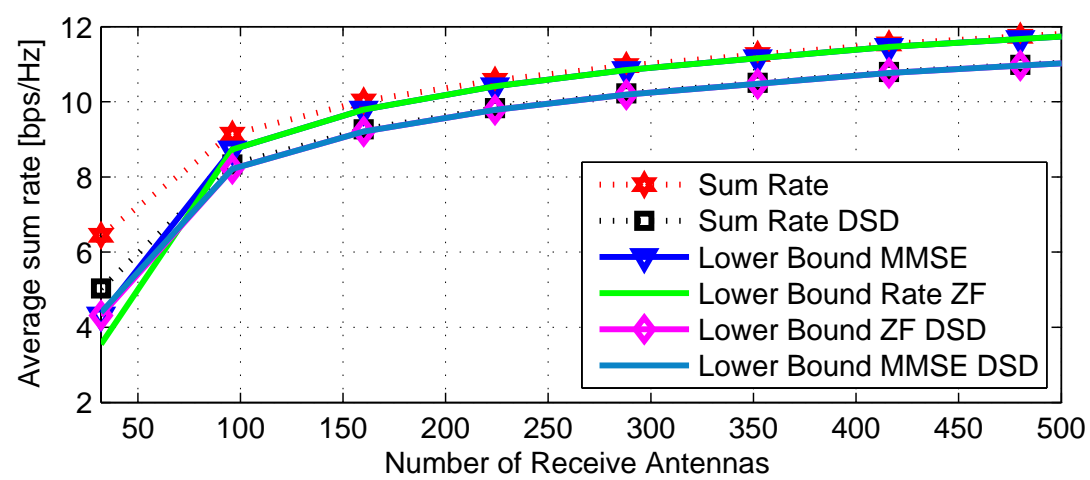

(a)

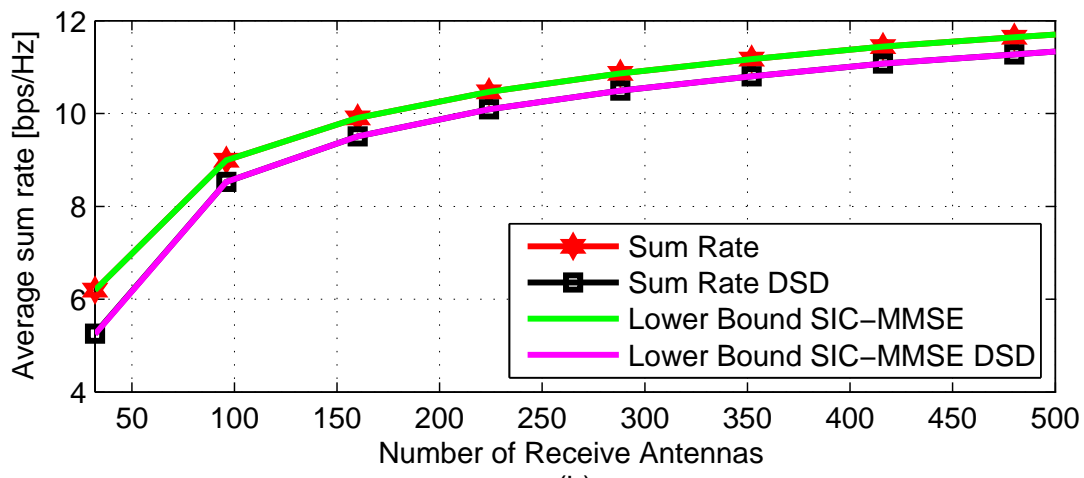

(b)

Figure 5.3: (a) Sum Rate vs Number of Receive antennas. $N=3,\left|C_{n}\right|=10$ users per class, $N_{t_{k, n}}=1$ antennas per user. (b) Sum Rate vs Number of Receive antennas. $N=2,\left|C_{n}\right|=16$ users per class, $N_{t_{k, n}}=1$ antennas per user.

can see from the plot that the lower bounds for the proposed DSD algorithms are very close to the lower bounds when the detection procedure is carried out together for all users.

\section{5 .2}

\section{Computational Complexity Analysis}

In this subsection, the computational complexity of the proposed DSD algorithm is evaluated and compared with the traditional coupled detection schemes, when all user classes are detected together, by counting the number of floating point operations (FLOPs) per received vector $\mathbf{y}$. Different detection schemes are considered such as MMSE, SIC and MB-SIC. We consider the MB-SIC detector because it has a high computational complexity, and with this we can analyze the behavior of the proposed DSD scheme when it is combined with detectors that require high computational cost. The SIC based receivers all use MMSE detection. Furthermore, the single-branch SIC and the first branch of the MB-SIC employ norm-based ordering. We consider QPSK modulation, however the computational cost in these detectors does 


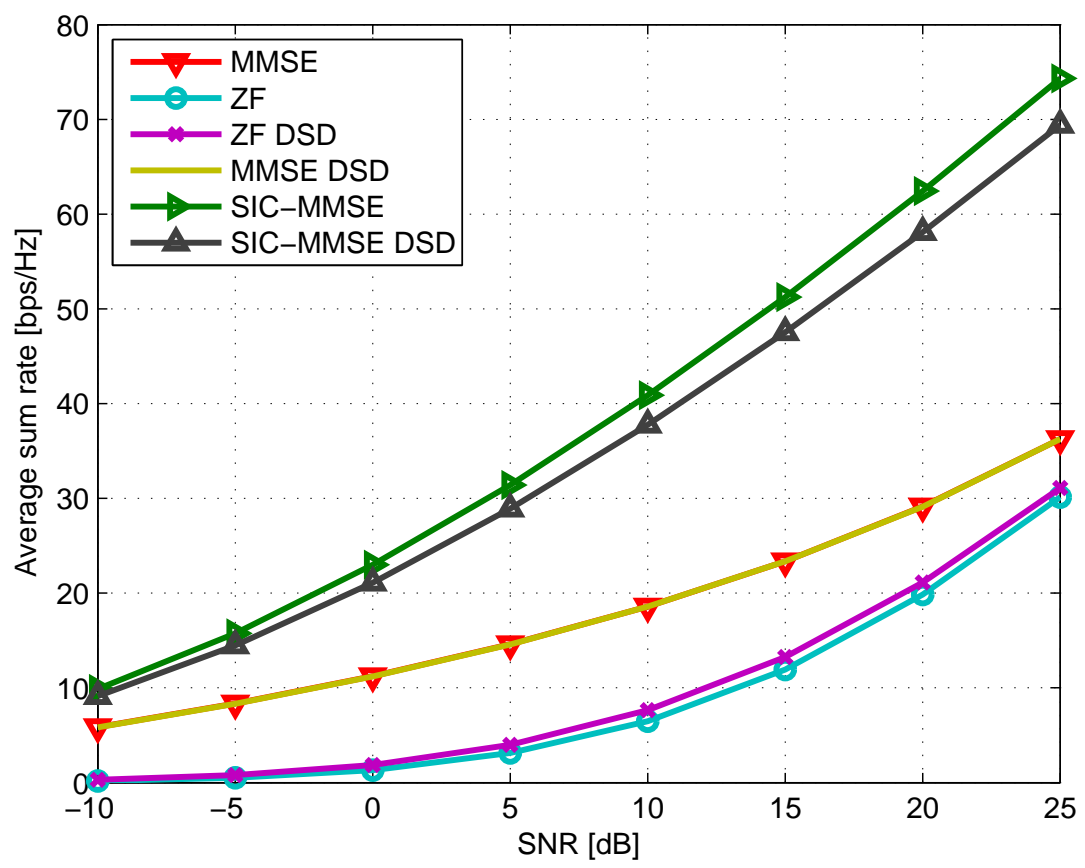

Figure 5.4: Sum Rate vs SNR. N=8, $\left|C_{n}\right|=1$ users per class, $N_{t_{k, n}}=8$ antennas per user. We consider DAS configuration with $N_{B}=96, D=4$ and $Q=8$.

not change significantly with the modulation order. The number of FLOPs for the complex QR decomposition of an $N_{t_{n}} \times N_{r}$ matrix is given in [99] as $16\left(N_{r}^{2} N_{t_{n}}-N_{t_{n}}^{2} N_{r}+1 / 3 N_{t_{n}}^{3}\right)$. To compute the number of FLOPs required for the remaining operations, we use the Light-speed Matlab toolbox [83].

Fig. 5.5 shows the computational complexity versus the number of user classes for different detection algorithms. We consider $K=100$ active users, $N_{t_{k, n}}=2$ transmit antennas per user and $N_{r}=200$ receive antennas distributed around the cell. We consider an increasing number of classes of users, when $K$ is not divisible by the number of classes, the number of active users is set to a smaller value so as to allow the division in $N$ classes. We can see from the figure that the complexity of the SIC and the MB-SIC detectors with the DSD algorithm is lower than the SIC and the MB-SIC coupled detectors, respectively. Furthermore, for receivers with DSD the computational complexity is reduced as the number of user classes is increased. This fact represents an important advantage for receivers with DSD, due to the fact that it allows to use of more complex detectors for each user class according to its data requirements.

In Fig. 5.6 and Fig. 5.7 we plot the required number of FLOPs versus the number of active users and versus the number of antennas per user, respectively. For Fig. 5.6 we consider $N=5$ classes of users, $N_{t_{k, n}}=2$ transmit 


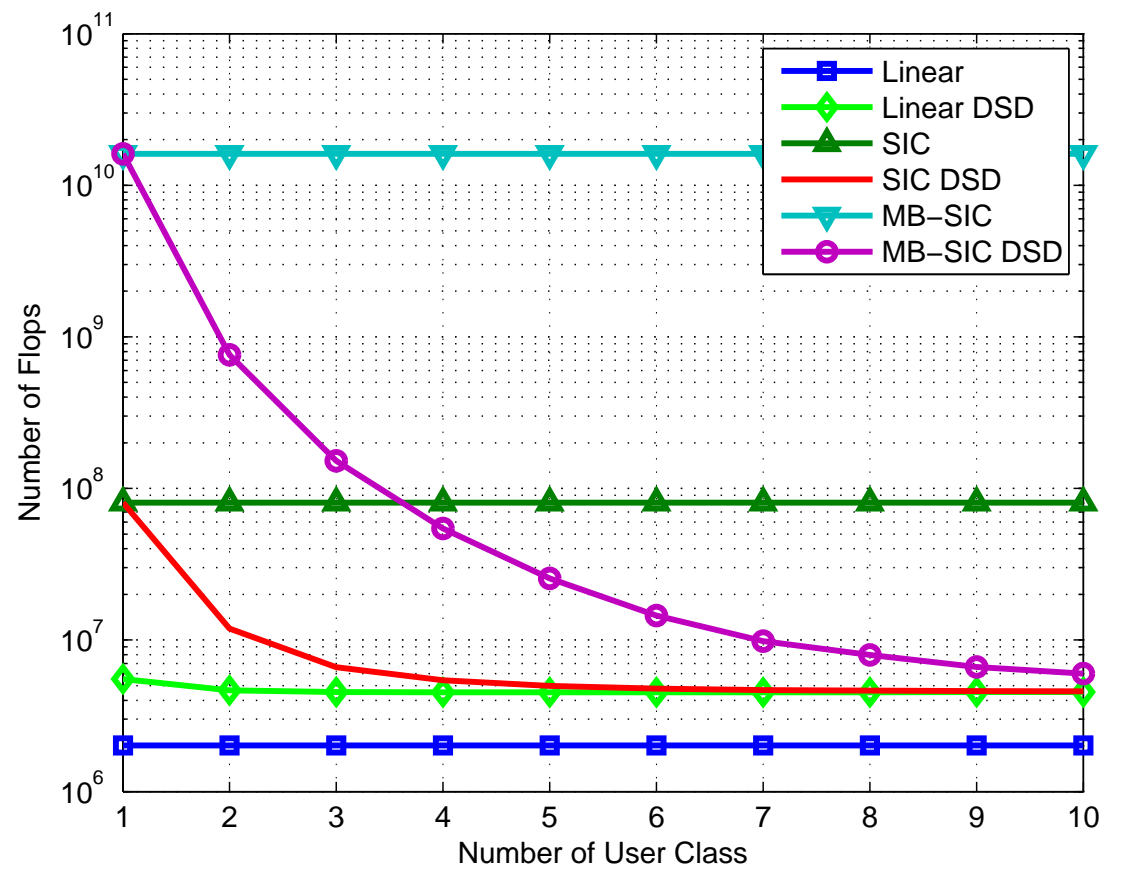

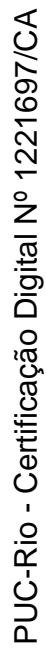

Figure 5.5: Computational complexity versus number of user class, $K=100$ active users, $N_{t_{k, n}}=2$ antennas per user, $N_{r}=200$ receive antennas.

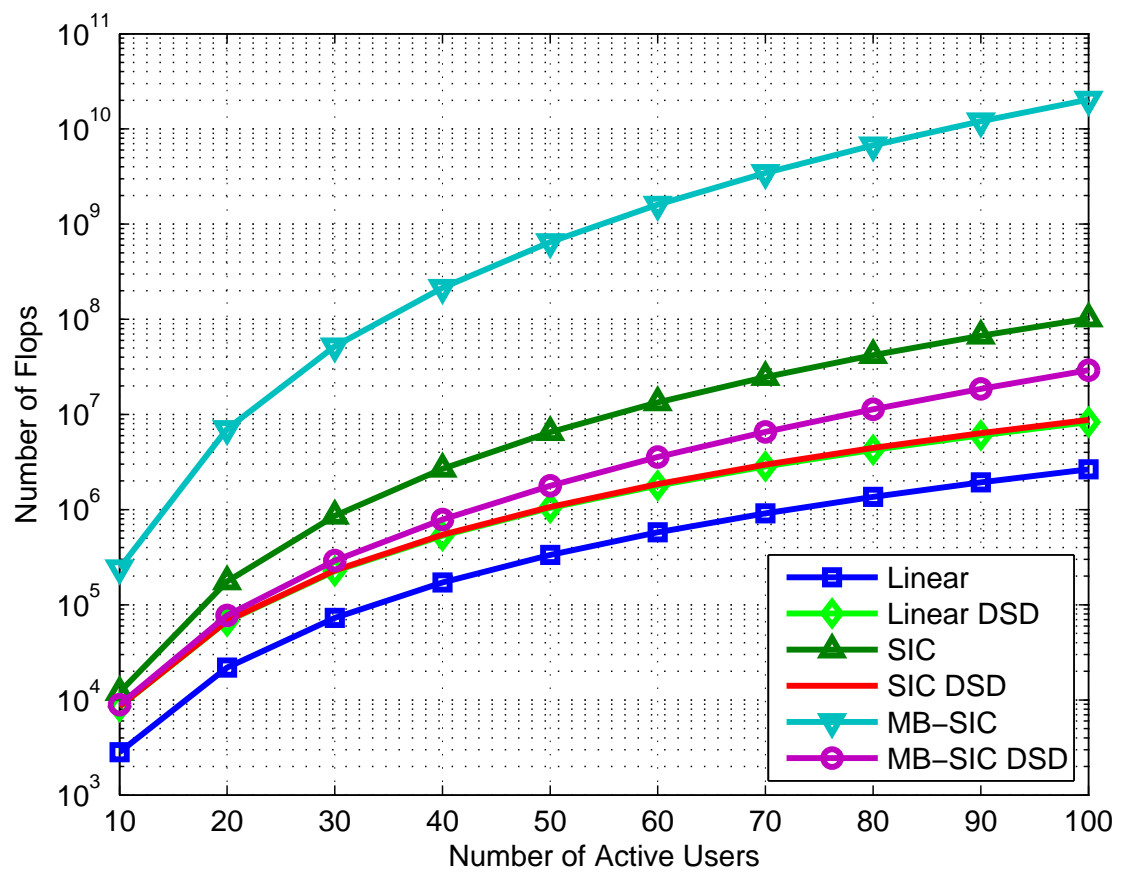

Figure 5.6: Computational complexity versus number of active user, $N=5$ classes of user, $N_{t_{k, n}}=2$ antennas per user, $N_{t}=K N_{t_{k, n}}$ transmit antennas and $N_{r}=3 N_{t}$ receive antennas. 


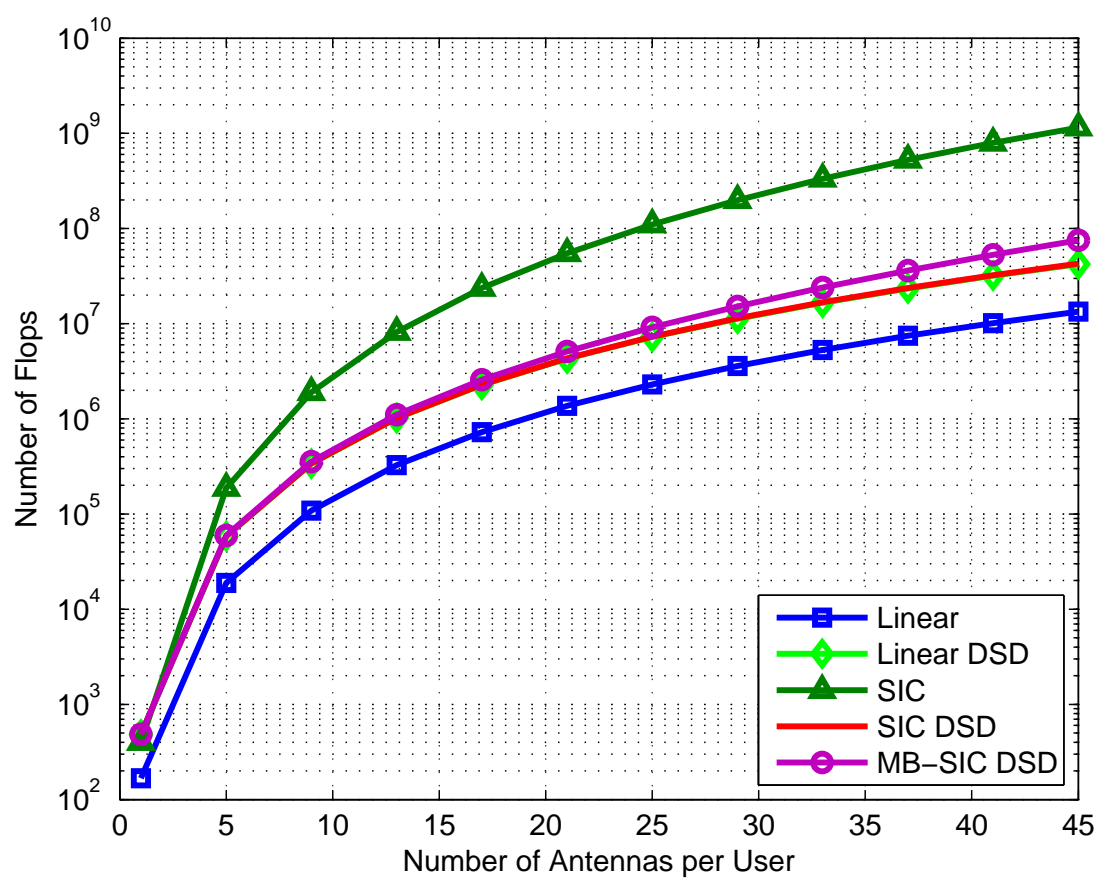

Figure 5.7: Computational complexity versus number of antennas per user, $K=10$ active user, $N=10$ classes of users, $N_{t}=K N_{t_{k, n}}$ transmit antennas and $N_{r}=2 N_{t}$ receive antennas.

antennas per user and $N_{r}=3 K N_{t_{k, n}}$ receive antennas. The MB-SIC and SIC detectors with DSD has a lower computational cost than the coupled SIC detector. For this $5 \mathrm{G}$ context, with a high number of antennas, efficient coupled detectors are not feasible to be implemented, however, if a specific user class requires the benefits of complex detectors, the DSD algorithm reduces the cost so that more complex detectors could be applied as illustrated with MB-SIC in Fig. 5.6. For the results in Fig. 5.7 we consider that we have $K=10$ active users and that we need to detect each user independently, i.e., $N=10$. The number of transmit antennas per user $N_{t_{k, n}}$ is increased. We also consider that the number of receive antennas distributed around the cell is given by $N_{r}=2 K N_{t_{k, n}}$. The MB-SIC and SIC detectors with DSD have a significantly lower complexity when compared with the SIC detector where all users are coupled.

It is worth noting that the curves displayed in Fig. 5.5, Fig. 5.6 and Fig. 5.7 will have a substantial decrease if the channel does not change over a time period due to quasi static channels. In this case, the equivalent channel matrices for each user class are stored for subsequent use. It would increase the gap, in terms of the computational cost, for the detection schemes using the DSD algorithm. 


\subsection{3 \\ BER Performance}

In this subsection, the BER performance of the proposed DSD algorithm is evaluated using different detectors which includes linear, SIC and MB-SIC with linear MMSE receive filters. The SIC detector of [34] uses a norm-based cancellation ordering, the MB-SIC of [37] employs a fixed number of branches, equal to the total number of transmit antennas per user class $N_{t_{n}}$ for the DSD schemes, and norm-based ordering in its first branch. A massive MIMO system operating in heterogeneous networks with $K$ active users is considered. We also consider the DAS configuration where the $N_{r}=N_{B}+D Q$ receive antennas are distributed around the cell in $D$ radio heads with $Q$ antennas each and the remaining $N_{B}$ antennas are located at the BS. We consider QPSK modulation. The SNR per transmitted information bit is defined as

$$
\mathrm{SNR}=10 \log _{10} \frac{N_{t} \sigma_{s}^{2}}{M \sigma_{n}^{2}},
$$

where $\sigma_{s}^{2}$ is the common variance of the transmitted symbols, $\sigma_{n}^{2}$ is the noise variance at the receiver and $M$ is the number of transmitted bits per symbol. The numerical results correspond to an average of 3,000 simulations runs, with $500 N_{t}$ symbols transmitted per run. For the $N_{B}$ antennas at the BS, we employ $L_{k}=0.3, \tau=2$, the distance to the users is obtained from a uniform discrete random variable distributed between 0.4 and 0.7 , the shadowing spread is $\sigma_{k}=1 \mathrm{~dB}$ and the transmit and receive correlation coefficients are equal to $\rho_{r x}=0.4$. For the $D$ remote arrays of antennas, we use $L_{k, j}$ taken from a uniform random variable distributed between 0.3 and 0.5 , the shadowing spread $\sigma_{k, j}=1$ $\mathrm{dB}$ and the receive correlation coefficients are equal to $\rho_{r x}=0.5$. When the number of transmit antennas at the users is $N_{t_{k, n}}>1$, the transmit correlation coefficient is equal to $\rho_{t x}=0.55$.

For the experiment in Fig. 5.8, we consider $K=12$ user devices, where each user is equipped with $N_{t_{k, n}}=3$ transmit antennas and $N=3$ classes of users. The system employs perfect channel state information and QPSK modulation. For the DAS configuration, we consider $N_{B}=8$ receive antennas at the BS, $D=4$ remote radio heads and $Q=7$ receive antennas per remote radio head. We can see from the figure that the decoupled SIC detection presents a performance close to the coupled SIC detector with a difference around $2 \mathrm{~dB}$ in the high SNR region. In addition the decoupled SIC detector has a drastic reduction in the computational cost when compared with its coupled version. The result also indicates a remarkable superiority in the performance for the MB-SIC receiver with the DSD scheme over the coupled SIC detector which also has a lower computational complexity than the coupled SIC detector. 


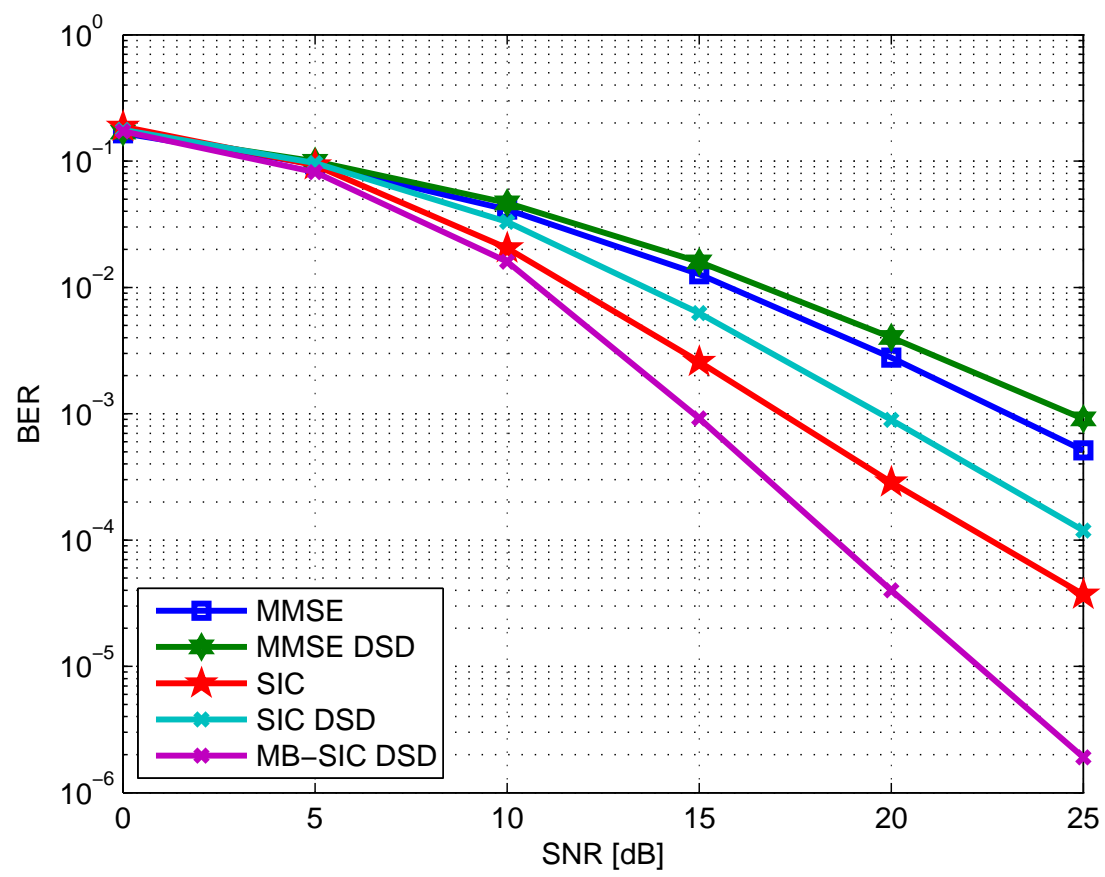

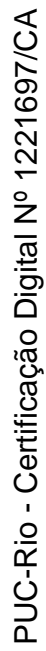

Figure 5.8: BER versus SNR with $K=12$ active users, $N=3$ classes of users, $N_{t_{k, n}}=3$ transmit antennas per user and $N_{r}=36$ receive antennas.

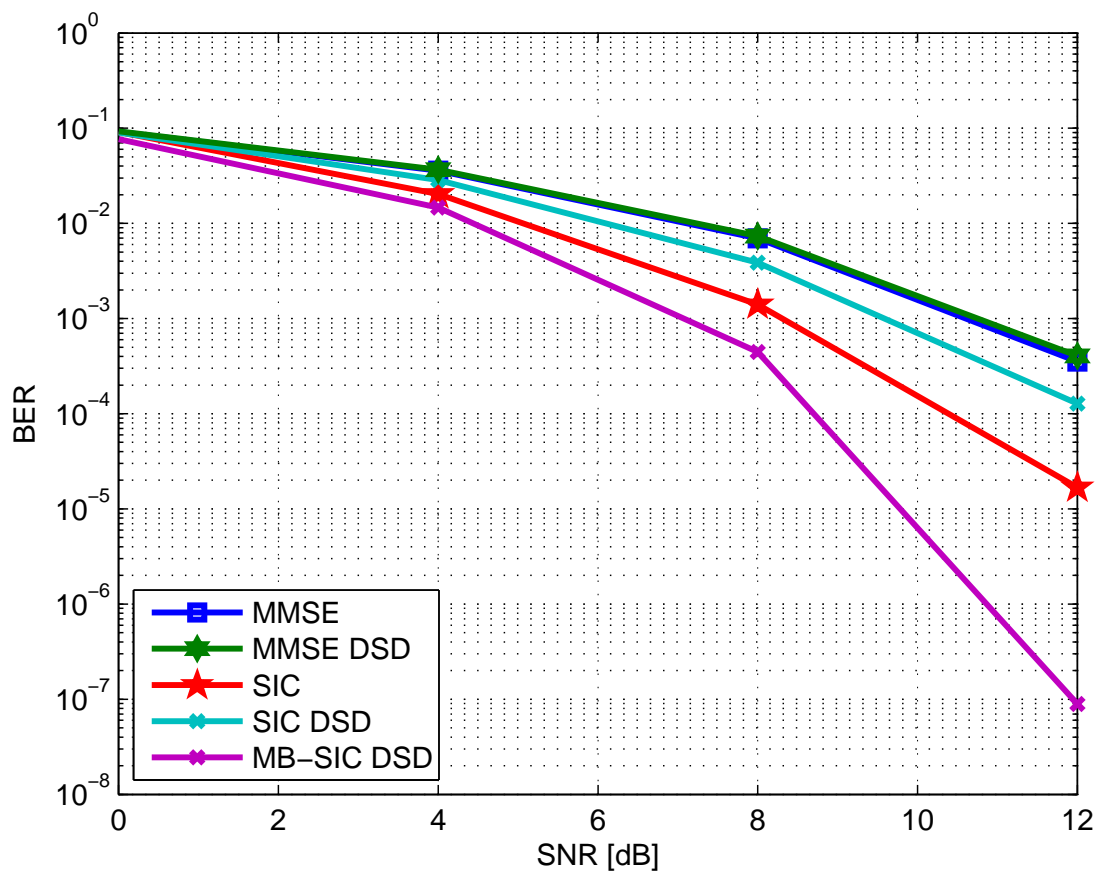

Figure 5.9: BER versus SNR with $K=8$ active users, $N_{t_{k, n}}=8$ transmit antennas per user, $N=8$ classes of users and $N_{r}=128$ receive antennas. 


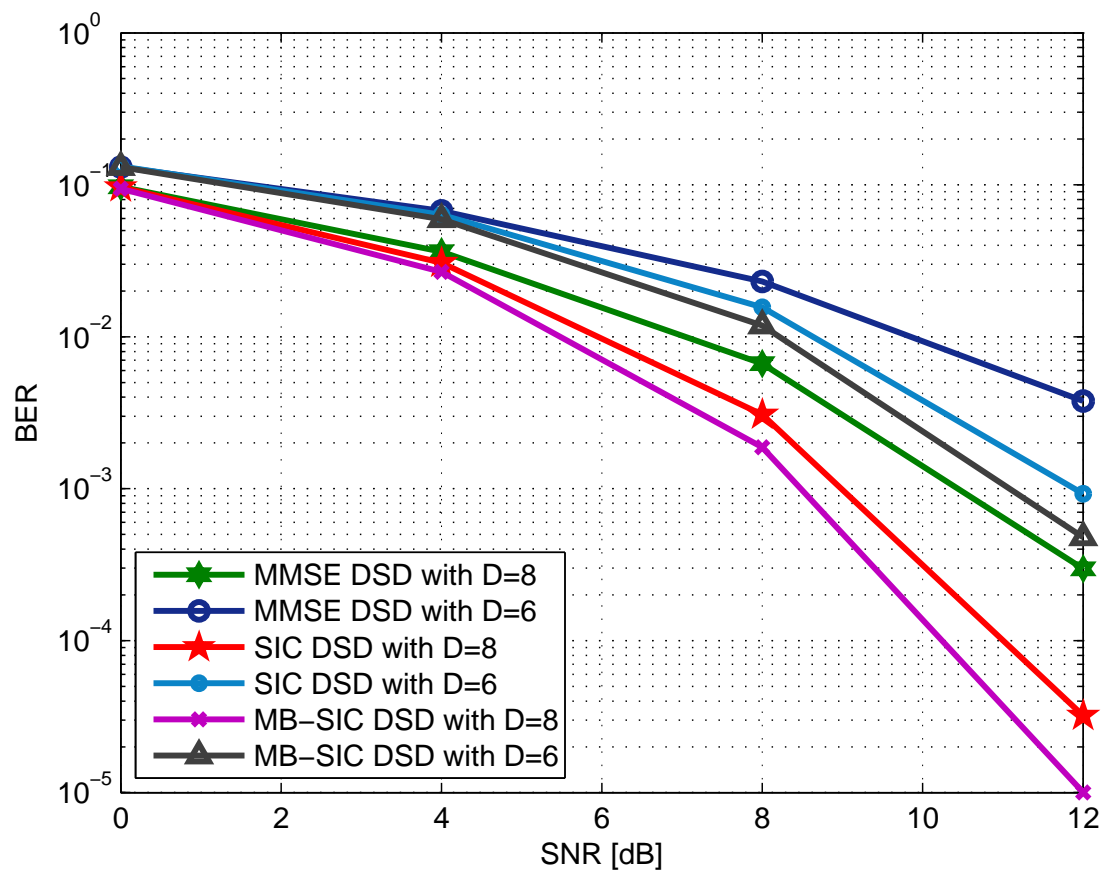

Figure 5.10: BER versus SNR with $K=64$ active users, $N_{t_{k, n}}=1$ transmit antennas per user, $N=4$ classes of users, $N_{B}=34, D$ remote antennas arrays with $Q=7$ receive antennas per array.

In the next example, we evaluate the performance of the proposed DSD scheme considering $K=8$ active users, each user transmitting with $N_{t_{k, n}}=8$ antennas. We also consider that we need to detect each user independently of each other, i.e., $N=8$ classes of users with $\left|C_{n}\right|=1$ users per class. For the DAS configuration, we consider $N_{B}=64$ receive antennas at the BS, $D=8$ remote radio heads and $Q=8$ receive antennas per remote radio head. The Fig. 5.9 indicate that the performance of the SIC detector with DSD is close to the SIC detector with a lower computational complexity. Note that the results for MB-SIC with DSD show a very good performance with a computational complexity much lower than the SIC detector without DSD.

In Fig. 5.10 we evaluate the performance of the proposed DSD scheme with MMSE, SIC and MB-SIC detection. We consider $K=64$ active users, $N=4$ classes of users, $\left|C_{n}\right|=16$ users per class and $N_{t_{k, n}}=1$ transmit antennas per user. For the DAS configuration, we consider $N_{B}=34$ receive antennas at the $\mathrm{BS}$ and $Q=7$ receive antennas per remote radio head. To show the behavior of the BER performance with different numbers of distributed antennas we consider two configurations of remote radios heads, $D=8$ and $D=6$. As expected, the results shows that when the number of RRHs is increased, the BER performance is improved due to the low propagation effects caused by 
the short distances between users and some remote antenna arrays. We also can see from the figure that the SIC and the MB-SIC detector with DSD offers an excellent BER performance with a low computational cost. 


\section{Conclusions and Future Works}

\section{1}

\section{Conclusions}

This thesis focused on aspects of design and implementation of efficient detection schemes for wireless communications systems with multiple antennas and users. Some detection techniques such as the multiple-branch lattice reduction successive interference cancellation (Chapter 3), variable list detection (Chapter 3) and iterative list detection and decoding (Chapter 4) have been proposed. A mathematical signal model and a decoupled signal detection scheme for heterogeneous networks (Chapter 5), have also been proposed and evaluated. The main outcomes of this thesis are techniques to achieve higher spectral efficiency using advanced interference cancellation for $5 \mathrm{G}$ environments.

In the following, we summarize the work in this thesis in terms of the content of the chapters.

General principles for multiuser MIMO systems have been studied in Chapter 2. Initially, we have reviewed and explained the capacity of MIMO systems in both deterministic and random channels. It was shown that the capacity can be increased by the factor $\min \left(N_{t}, N_{r}\right)$ when compared to a conventional single-antenna system. Channel estimation aspects and the most important detection techniques in the literature such as linear detectors (ZF, MMSE), successive interference cancellation based detectors (SIC, MB-SIC, MF-SIC) and the optimal ML detector have been presented.

In order to design efficient detectors in terms of BER performance and computational complexity for cellular networks operating with MU-MIMO systems, Lattice Reduction (LR) detection techniques have been presented in Chapter 3. Initially, the LR concept and some detection techniques in the LR domain have been studied. An efficient implementation for the LR-SIC detector was proposed as well as two detection schemes by list generation. The proposed MB-LR-SIC detector employs an LR transformation technique on the channel matrix, which was previously estimated, and generates a fixed number of multiple ordering patterns and estimates of symbol vectors, each 
of these ordering patterns uses SIC detection in the LR domain. This makes it possible to generate a set of candidate estimates of the transmitted vector. Finally the best candidate is selected using the ML criterion. The MB-LR-SIC detector has shown, through simulations, a good performance, however, due to the its relatively high computational complexity caused by the fixed number of candidates tested, MB-LR-SIC detector is not viable for high number of antennas scenarios. In Chapter 3, we have also proposed the VLD signal detection algorithm. The proposed VLD employs an LR transformation and makes a multistage search for a reliable estimation of the transmitted signal vector in the LR domain. In each stage, the proposed algorithm consider different ordering patterns for the LR-SIC detection. For the fist stage, an ordering that minimize the MSE between the transmitted symbol and the detected symbol is used as a starting point. Based on a quality metric of the current solution, a number of candidates necessary to find a better estimation is calculated using a random ordering pattern after the fist stage. The entire multistage search process is performed in the LR domain. Numerical results show that the proposed VLD performance results in a near optimal performance with much lower computational complexity than the ML detector. VLD offers a higher detection diversity and low computation complexity, compared with the optimal detector, the MB-LR-SIC algorithm and other existing schemes.

In Chapter 4, an iterative detection and decoding structure for Pointto-Point MIMO systems has been described. Moreover, we have developed an iterative version of the proposed VLD algorithm for MU-MIMO coded system. IVLDD uses the soft bit extrinsic information exchanged between the detector and the decoder through iterations. In the first iteration, reliable values of LLRs are computed using VLD which produces a good performance since the first iteration that reduces the required number of iterations. After the first iteration, the SC-MMSE is used to eliminate the remaining interference. For coded systems, IVLDD has shown that only three turbo iterations are needed to get close to the interference-free single-user performance.

In Chapter 5, a mathematical signal model and the DSD algorithm for the uplink of massive MIMO systems operating in heterogeneous cellular networks with different classes of users using CAS and DAS configurations has been proposed. The proposed DSD allows one to separate the received signals for each category of users efficiently into independent parallel single user class signals at the receiver side, applying a common channel inversion and QR decomposition and assuming that the channel matrix was previously estimated. 
With the proposed DSD scheme, it is possible to handle different classes of users in heterogeneous networks and to use different modulation and/or detection schemes for each class according to its data service requirements. The main advantage of DSD is the reduction in the computational cost of efficient detection schemes that, for its high computational complexity, are not viable to be implemented when the signals received from all active users are coupled.

\section{2}

\section{Future Work}

The techniques described in this thesis have considered a single cell scenario, however, some extra challenges could appear in multicell environments due to the inter-cell interference. The proposed schemes may be generalized to a multicell scenario. On the other hand, the receiver proposed in Chapters 3-5 could be extended to transmission using orthogonal frequency division multiplexing techniques.

Some suggestions for possible future works based on this thesis are given bellow:

- To consider multicell MIMO systems.

- To analyze the synchronization problem in the communications networks studied here.

- To develop an iterative detection and decoding technique for heterogeneous networks.

- To improve the error control coding. Stronger codes such as turbo codes and LDPC can be used instead of convolutional codes used in this thesis.

- To consider cooperative signal relaying for massive MIMO systems in heterogeneous networks.

- Hardware implementation of the receiver structures proposed in Chapters 3-5. 


\section{Bibliography}

[1] International Telecommunication Union (ITU), "Measuring the information society report 2015," December, 2015. [Online]. Available: http://www.itu.int/en/ITU-D/Statistics/Documents/ publications/misr2015/MISR2015-w5.pdf

[2] Ericsson, "Ericsson mobility report, on the pulse of the networked society," November, 2015. [Online]. Available: http://www.ericsson.com/ res/docs/2015/mobility-report/ericsson-mobility-report-nov-2015.pdf

[3] P. Demestichas, A. Georgakopoulos, D. Karvounas, K. Tsagkaris, V. Stavroulaki, J. Lu, C. Xiong, and J. Yao, "5G on the Horizon: Key Challenges for the Radio-Access Network," IEEE Vehicular Technology Magazine, vol. 8, no. 3, pp. 47 - 53, September 2013.

[4] T. Tjelta, G. Millstein, O. Grondalen, O. Osterbo, P. H. Lehne, P. Waldemar, P. Gronsund, K. Mahmood, and H. Lonsethagen, "Research topics and initial results for the fifth generation (5G) mobile network," 1 st International Conference on $5 G$ for Ubiquitous Connectivity (5GU), pp. 267-272, 2014.

[5] D. Liu, L. Wang, Y. Chen, M. Elkashlan, K. Wong, R. Schober, and L. Hanzo, "User Association in 5G Networks: A Survey and an Outlook," IEEE Communications Surveys and Tutorials, no. 99, 2016.

[6] P. Patcharamaneepakorn, S. Wu, C. Wang, M. Aggoune, M. M. Alwakeel, X. Ge, and M. D. Renzo, "Spectral, Energy and Economic Efficiency of 5G Multi-cell Massive MIMO Systems with Generalized Spatial Modulation," IEEE Transactions on Vehicular Technology, no. 99, 2016.

[7] X. Ge, J. Ye, Y. Yang, and Q. Li, "User Mobility Evaluation for 5G Small Cell Networks Based on Individual Mobility Model," IEEE Journal on Selected Areas in Communications, vol. 34, no. 3, 2016.

[8] T. E. Bogale and L. B. Le, "Massive MIMO and mmWave for 5G Wireless HetNet: Potential Benefits and Challenges," IEEE Vehicular Technology Magazine, vol. 11, no. 1, pp. 64-75, 2016.

[9] 3GPP-LTE, "Technical specification group radio access network: Evolved universal terrestrial radio access (E-UTRA): further advancements for EUTRA physical layer aspects (release 9)," 3GPP TR36.814, 2010. 
[10] M. K. Iqbal, M. B. Iqbal, I. Rasheed, and A. Sandhu, " 4 G Evolution and Multiplexing Techniques with Solution to Implementation Challenges," International Conference on Cyber-Enabled Distributed Computing and Knowledge Discovery (CyberC), vol. 11, no. 1, pp. 485-488, 2012.

[11] M. Corici, M. Constantin, D. Satriya, D. Vingarzan, V. Vlad, and L. Wollner, "Integrating off-the-shelf 3GPP access networks in the OpenEPC software toolkit: Realizing cost-efficient and complete small-scale operator testbeds," IEEE Globecom Workshops (GC Wkshps), pp. 17241729, 2012.

[12] C. B. Sankaran, "Data offloading techniques in 3GPP Rel-10 networks: A tutorial," IEEE Communications Magazine, vol. 50, no. 6, pp. 46-53, 2012.

[13] C. Gentner, E. Munoz, M. Khider, E. Staudinger, S. Sand, and A. Dammann, "Particle Filter Based Positioning with 3GPP-LTE in Indoor Environments," IEEE Position Location and Navigation Symposium (PLANS), pp. 301-308, 2012.

[14] S. Alamouti, "A simple transmit diversity technique for wireless communications," IEEE Journal on Selected Areas in Communications, vol. 16, no. 8, pp. $1451-1458,1998$.

[15] C. J. Foschini and M. J. Gans, "On limits of wireless communications in a fading enviroment when using multiple antennas," Wireless Pers. Commun., vol. 6, pp. $311-335,1998$.

[16] I. E. Telatar, "Capacity of Multi-Antenna Gaussian channels," European Transactions on Communications, vol. 10, no. 6, pp. 585 - 595, 1999.

[17] N. Chiurtu, B. Rimoldi, and E. Telatar, "On the capacity of multiantenna Gaussian channels," in IEEE International Conference on Information Theory, Washington, United States, 2001, p. 53.

[18] T. L. Marzetta, "Noncooperative cellular wireless with unlimited numbers of base station antennas," IEEE Transactions on Wireless Communications, vol. 9, no. 11, pp. 3590 - 3600, November 2010.

[19] E. G. Larsson, O. Edfors, F. Tufvesson, and T. L. Marzetta, "Massive MIMO for next generation wireless systems," IEEE Communications Magazine, vol. 52, no. 2, pp. 186-195, 2014. 
[20] F. Rusek, D. Persson, B. Lau, E. Larsson, T. Marzetta, O. Edfors, and F. Tufvesson, "Scaling up MIMO: Opportunities, and challenges with very large arrays," IEEE Signal Processing Magazine, vol. 30, no. 1, pp. 40 - 60, January 2013.

[21] R. C. de Lamare, "Massive MIMO Systems: Signal Processing Challenges and Future Trends," URSI Radio Science Bulletin, December 2013.

[22] U. Ugurlu, R. Wichman, C. Ribeiro, and C. Wijting, "A Multipath Extraction Based CSI Acquisition Method for FDD Cellular Networks with Massive Antenna Arrays," IEEE Transactions on Wireless Communications, no. 99, pp. 1-14, 2015.

[23] D. Zhu, B. Li, and P. Liang, "On the matrix inversion approximation based on neumann series in massive MIMO systems," IEEE International Conference on Communications (ICC), pp. 1763-1769, 2015.

[24] H. Wang, X. Ge, R. Zi, J. Zhang, and Q. Ni, "Multimedia over massive MIMO wireless systems," International Wireless Communications and Mobile Computing Conference (IWCMC), pp. 1492-1497, 2015.

[25] X. Gao, O. Edfors, F. Rusek, and F. Tufvesson, "Massive MIMO Performance Evaluation Based on Measured Propagation Data," IEEE Transactions on Wireless Communications, vol. 14, no. 7, pp. 3899-3911, 2015.

[26] Y. Zhao, X. Wang, X. Gu, W. Wan, and Q. Pang, "Training sequence design for channel state information acquisition in massive MIMO systems," IEEE 26th Annual International Symposium on Personal, Indoor, and Mobile Radio Communications (PIMRC), pp. 1712-1716, 2015.

[27] T. Marzetta, "Noncooperative cellular wireless with unlimited numbers of base station antennas," IEEE Transactions on Wireless Communications, vol. 9, no. 11, pp. 3590 - 3600, 2010.

[28] E. G. Larsson, F. Tufvesson, O. Edfors, and T. L. Marzetta, "Massive MIMO for next generation wireless systems," IEEE Communication Magazine, vol. 52, no. 2, pp. 186 - 195, February 2014.

[29] U. Fincke and M. Pohst, "Improved methods for calculating vectors of short length in a lattice, including a complexity analysis," Mathematics of Computation, vol. 44, no. 170, pp. 463 - 471, 1985. 
[30] B. Hassibi and H. Vikalo, "On the sphere decoding algorithm I, Expected complexity," IEEE Transactions on Signal Processing, vol. 53, no. 8, pp. $2806-2818,2005$.

[31] J. Jalden and B. Ottersten, "On the Complexity of Sphere Decoding in Digital Communications," IEEE Transactions on Signal Processing, vol. 53, no. 4, pp. $1474-1484,2005$.

[32] B. Hassibi and H. Vikalo, "On the sphere decoding algorithm II. Generalizations, second order statistics, and applications to communications," IEEE Transactions on Signal Processing, vol. 53, no. 8, pp. 2819 - 2834, 2005.

[33] A. Paulraj, R. Nabar, and D. Gore, "Introduction to Space-Time Wireless Communications," Cambridge University Press, 2003.

[34] G. Golden, C. Foschini, R. Valenzuela, and P. Wolniansky, "Detection algorithm and initial laboratory results using V-BLAST space-time communication architecture," Electronics Letters, vol. 35, no. 1, pp. 14 - 16, January 1999.

[35] T. Liu, J. Jiang, and Y. Chu, "A Low-Cost MMSE-SIC Detector for the MIMO System: Algorithm and Hardware Implementation," IEEE Transactions on Circuits and Systems II, vol. 58, no. 1, pp. 56-61, 2011.

[36] R. de Lamare and R. Sampaio-Neto, "Minimum Mean-Squared Error Iterative Successive Parallel Arbitrated Decision Feedback Detectors for DS-CDMA Systems," IEEE Transactions on Communications, vol. 56, no. 5, pp. 778 - 789, May 2008.

[37] R. de Lamare, "Adaptive and Iterative Multi-Branch MMSE Decision Feedback Detection Algorithms for Multi-Antenna Systems," IEEE Transactions on Wireless Communications, vol. 12, no. 10, pp. 5294 5308, 2013.

[38] P. Li, R. C. de Lamare, and R. Fa, "Multiple Feedback Successive Interference Cancellation Detection for Multiuser MIMO Systems," IEEE Transactions on Wireless Communications, vol. 10, no. 8, pp. 2434 2439, 2011.

[39] M. Chiani, "Introducing erasures in decision-feedback equalization to reduce error propagation," IEEE Transactions on Communications, vol. 45 , no. 7 , pp. $757-760,1997$. 
[40] M. Reuter, J. C. Allen, J. R. Zeidler, and R. C. North, "Mitigating Error Propagation Effects in a Decision Feedback Equalizer," IEEE Transactions on Communications, vol. 49, no. 11, pp. 2028 - 72041 , 2001.

[41] E. Viterbo and J. Boutros, "A universal lattice code decoder for fading channels," IEEE Transactions on Information Theory, vol. 45, no. 5, pp. 1639 - 1642, 1999.

[42] M. Taherzadeh, A. Mobasher, and A. K. Khandani, "LLL Reduction Achieves the Receiver Diversity in MIMO Decoding," IEEE Transactions on Information Theory, vol. 53, no. 12, pp. 4801 - 4805, 2007.

[43] H. Yao and G. Wornell, "Lattice-reduction-aided detectors for MIMO communication systems," IEEE Global Telecommunications Conference, vol. 1, pp. $424-428,2002$.

[44] Y. H. Gan and W. H. Mow, "Complex lattice reduction algorithms for low-complexity MIMO detection," IEEE Global Telecommunications Conference, vol. 5, pp. 2953 - 2957, 2005.

[45] H. Napias, "A generalization of the LLL-algorithm over euclidean rings or orders," Journal de thorie des nombres de Bordeaux, vol. 8, no. 2, pp. $387-396,1996$.

[46] C. Windpassinger and R. F. H. Fischer, "Low-complexity nearmaximum-likelihood detection and precoding for MIMO systems using lattice reduction," Proc. of IEEE Information Theory Workshop (ITW), pp. $345-348,2003$.

[47] J. Park and J. Chun, "Efficient Lattice-Reduction-Aided Successive Interference Cancellation for Clustered Multiuser MIMO System," IEEE Transactions on Vehicular Technology, vol. 61, no. 8, pp. 3643 - 3655, October 2012.

[48] Q. Li, J. Zhang, L. Bai, and J. Choi, "Lattice Reduction-Based Approximate MAP Detection with Bit-Wise Combining and Integer Perturbed List Generation," IEEE Transactions on Communications, vol. 61, no. 8, pp. 3259 - 3269, August 2013.

[49] H. Najafi and M. O. Damen, "Lattice-Reduction-Aided Conditional Detection for MIMO Systems," IEEE Transactions on Communications, vol. 62, no. 11, pp. 3864 - 3873, November 2014. 
[50] J. Park, B. C. ans J. Chun, and B. J. Jeong, "Lattice Reduction-Aided Successive Interference Cancelation for MIMO Interference Channels," IEEE Transactions on Vehicular Technology, vol. 63, no. 8, pp. 4131 4135, October 2014.

[51] M. Neinavaie, A. R. Zolghadrasli, M. Derakhtian, and M. Zolghadrasli, "Diversity Analysis of Lattice-Reduction Aided Linear Equalizers in Decode and Forward MIMO Relay Networks," IEEE Transactions on Wireless Communications, vol. 13, no. 12, pp. 6593 - 6605, Dezember 2014.

[52] Y. H. Gan, C. Ling, and W. H. Mow, "Complex Lattice Reduction Algorithm for Low-Complexity Full-Diversity MIMO Detection," IEEE Transactions on Signal Processing, vol. 57, no. 7, pp. 2701 - 2710, July 2009.

[53] D. Wübben, R. Böhnke, V. Kühn, and K.-D. Kammeyer, "Nearmaximum-likelihood detection of MIMO systems using MMSE-based lattice-reduction," in IEEE International Conference on Communications, Paris, France, June 2004, pp. 798 - 802.

[54] X. Ma and W. Zhang, "Performance analysis for MIMO systems with lattice-reduction aided linear equalization," IEEE Transactions on Communications, vol. 56, no. 2, pp. 309 - 318, 2008.

[55] K. Lee, J. Chun, and L. Hanzo, "Optimal Lattice-Reduction Aided Successive Interference Cancellation for MIMO Systems," IEEE Transactions on Signal Processing, vol. 6, no. 7, pp. 2438 - 2443, 2007.

[56] T. Matsumoto, "Iterative (turbo) signal processing techniques for MIMO signal detection and equalization," in Smart Antennas in Europe - Stateof-the-Art, EURASIP Book Series, 2005.

[57] H. Pervaiz, L. Musavian, and Q. Ni, "Area energy and area spectrum efficiency trade-off in 5G heterogeneous networks," 2015 IEEE International Conference on Communication Workshop (ICCW), pp. 1178-1183, 2015.

[58] W. Roh and A. Paulraj, "MIMO channel capacity for the distributed antenna systems," IEEE Vehicular Technology Conference, vol. 3, pp. 1520 - 1524, September 2002.

[59] C. Loyez, M. Bocquet, C. Lethien, and N. Rolland, "A Distributed Antenna System for Indoor Accurate WiFi Localization," IEEE Antennas and Wireless Propagation Letters, vol. 14, pp. 1184-1187, 2015. 
[60] L. Dai, "An Uplink Capacity Analysis of the Distributed Antenna System (DAS): From Cellular DAS to DAS with Virtual Cells," IEEE Transactions on Wireless Communications, vol. 13, no. 5, pp. 2717-2731, May 2014.

[61] R. Heath, S. Peters, Y. Wang, and J. Zhang, "A current perspective on distributed antenna systems for the downlink of cellular systems," IEEE Communications Magazine, vol. 51, no. 4, pp. 161-167, 2013.

[62] J. Wang and L. Dai, "Asymptotic Rate Analysis of Downlink Multi-User Systems With Co-Located and Distributed Antennas," IEEE Transactions on Wireless Communications, vol. 14, no. 6, pp. 3046-3058, 2015.

[63] G. S. D. Gordon, M. J. Crisp, R. V. Penty, and I. H. White, "Experimental Comparison of Antenna Clustering Strategies in MIMO Distributed Antenna Systems," IEEE 80th Vehicular Technology Conference (VTC Fall), pp. 1-5, 2014.

[64] D. Wang, J. Wang, X. You, Y. Wang, M. Chen, and X. Hou, "Spectral Efficiency of Distributed MIMO Systems," IEEE Journal on Selected Areas in Communications, vol. 31, no. 10, pp. 2112 - 2127, October 2002.

[65] F. Römer, M. Fuchs, and M. Haardt, "Distributed MIMO systems with spatial reuse for highspeed indoor mobile radio access," 20th Meeting of the Wireless World Research Forum (WWRF), Ottawa, Canada, 2008.

[66] L. Arévalo, R. de Lamare, M. Haardt, and R. Sampaio-Neto, "Uplink Block Diagonalization for Massive MIMO-OFDM Systems with Distributed Antennas," 2015 IEEE International Workshop on Computational Advances in Multi-Sensor Adaptive Processing (CAMSAP), 2015.

[67] L. Arévalo, C. A. Medina, and R. Sampaio-Neto, "Playing with Blocks: A New Combination of Block Transmission and the CDMA Multiple Access Technique," IEEE 81st Vehicular Technology Conference (VTC Spring), pp. 1-4, May 2015.

[68] L. Arévalo, R. de Lamare, and R. Sampaio-Neto, "Iterative MultiBranch Lattice Reduction-Aided Successive Interference Cancellation for Multiuser MIMO Systems," 19th International ITG Workshop on Smart Antennas (WSA), pp. 1-5, 2015. 
[69] L. Arévalo, R. de Lamare, K. Zu, and R. Sampaio-Neto, "Multi-branch lattice reduction successive interference cancellation detection for multiuser MIMO systems," IEEE International Symposium on Wireless Communications Systems, pp. 219 - 223, August 2014.

[70] L. Arévalo, J. A. Apolinario, M. L. R. de Campos, and R. Sampaio-Neto, "Convex Combination of Three Affine Projections Adaptive Filters," IEEE International Symposium on Wireless Communications Systems (ISWCS), pp. 1-5, 2013.

[71] R. Knopp and P. Humblet, "Information capacity and power control in single-cell multiuser communications," in IEEE International Conference on Communications, vol. 11, Seatle, United States, 1995, pp. 331 - 335.

[72] M. Bengtsson, "From single link MIMO to multi-user MIMO," in IEEE International Conference on Acoustics, Speech, and Signal Processing, vol. 4, Montreal, Canada, May 2004, pp. iv-697 - iv-700.

[73] E. Karami, "Tracking Performance of Least Squares MIMO Channel Estimation Algorithm," IEEE Trans. Commun., vol. 55, no. 11, pp. 2201 - 2209, Nov. 2007.

[74] C. Pietsch, S. Sand, W. G. Teich, and J. Lindner, "Modeling and performance evaluation of multiuser MIMO systems using real-valued matrices," IEEE Journal on Selected Areas in Communications, vol. 21, no. 5, pp. $744-753,2003$.

[75] R. Fa and R. C. de Lamare, "Design of Adaptive Multi-Branch SIC Receivers for MIMO Spatial Multiplexing Systems," in International Symposium on Wireless Communication Systems (ISWCS), Siena-Tuscany, Italy, 2009, pp. $575-579$.

[76] D. Shiu, G. J. Foschini, M. J. Gans, and J. M. Kahn, "Fading correlation and its effect on the capacity of multielement antenna systems," IEEE Transactions on Communications, vol. 48, no. 3, pp. 502 - 513, 2000.

[77] M. Taherzadeh, A. Mobasher, and A. K. Khandani, "Lattice-basis reduction achieves the precoding diversity in MIMO broadcast systems," in Proc. 39th Conf. on Information Sciences and Systems, Johns Hopkins Univ., USA, March 15-18, 2005.

[78] D. Milford and M. Sandell, "Simplified Quantisation in a ReducedLattice MIMO Decoder," IEEE Communications Letters, vol. 15, no. 7, pp. 725 - 727, July 2011. 
[79] A. A. Pereira-Jr and R. Sampaio-Neto, "A Random-List Based LAS Algorithm for Near-Optimal Detection in Large-Scale Uplink Multiuser MIMO Systems," 19th International ITG Workshop on Smart Antennas WSA, 3-5 March 2015.

[80] J. Cal-Braz and R. Sampaio-Neto, "Projection-Based List Detection in Generalized Spatial Modulation MIMO Systems," IEEE Communications Letters, vol. 19, no. 7, pp. 1145 - 1148, July 2015.

[81] J. Kermoal, L. Schumacher, and K. P. et al., "A stochastic MIMO radio channel model with experimental validation," IEEE J. Sel. Areas Commun., vol. 20, no. 6, pp. 1211 - 1226, Aug. 2002.

[82] H. Vetter, V. Ponnampalam, M. Sandell, and P. A. Hoeher, "Fixed Complexity LLL Algorithm," IEEE Transactions on Signal Processing, vol. 57, no. 4, pp. $1634-1637$, April 2009.

[83] T. Minka, "The Lightspeed Matlab toolbox, efficient operations for Matlab programming, version 2.2," Microsoft Corp, 17 Dec. 2007.

[84] J. Hagenauer, E. Offer, and L. Papke, "Iterative decoding of binary block and convolutional codes," IEEE Transactions on Information Theory, vol. 42, no. 2, pp. 429 - 445, 1996.

[85] M. Honig, G. Woodward, and Y. Sun, "Adaptive iterative multiuser decision feedback detection," IEEE Transactions on Wireless Communications, vol. 3, no. 2, pp. 477 - 485, 2004.

[86] P. D. Alexander, M. C. Reed, J. Asenstorfer, and C. B. Schlegel, "Iterative multiuser interference reduction: turbo CDMA," IEEE Transactions on Communications, vol. 47, no. 7, pp. 1008 - 1014, 1999.

[87] X. Wang and H. Poor, "Iterative (Turbo) soft interference cancellation and decoding for coded CDMA," IEEE Transactions on Communications, vol. 47, no. 7, pp. 1046 - 1061, 1999.

[88] C. Studer, "Iterative MIMO decoding: algorithms and VLSI implementation aspects," PhD thesis Hartung-Gorre Publisher, 2009.

[89] H. Poor and S. Verdú, "Probability of error in MMSE multiuser detection," IEEE Transactions on Information Theory, May 1997.

[90] Q. H. Spencer, A. L. Swindlehurst, and M. Haardt, "Zero-forcing methods for downlink spatial multiplexing in multiuser MIMO channels," 
IEEE Transactions on Signal Processing, vol. 52, no. 2, pp. 461 - 471, February 2004.

[91] L. U. Choi and R. D. Murch, "A transmit preprocessing technique for multiuser MIMO systems using a decomposition approach," IEEE Transactions on Wireless Communications, vol. 3, no. 1, pp. $20-24$, January 2004.

[92] V. Stankovic and M. Haardt, "Generalized design of multi-user MIMO precoding matrices," IEEE Transactions on Wireless Communications, vol. 7 , no. 3, pp. 953 - 961, March 2008.

[93] C. B. Chae, S. Shim, and R. W. Heath, "Block diagonalized vector perturbation for multiuser MIMO systems," IEEE Transactions on Wireless Communications, vol. 7, no. 11, pp. 4051 - 4057, November 2008.

[94] V. Stankovic and M. Haardt, "Improved diversity on the uplink of multiuser MIMO systems," 2005 European Conference on Wireless Technology (EuWiT), pp. 113-116, 2005.

[95] K. Zu, R. C. de Lamare, and M. Haardt, "Generalized Design of Low-Complexity Block Diagonalization Type Precoding Algorithms for Multiuser MIMO Systems," IEEE Transactions on Communications, vol. 61, no. 10, pp. 4232-4242, 2013.

[96] H. Sung, S. Lee, and I. Lee, "Generalized Channel Inversion Methods for Multiuser MIMO Systems," IEEE Transactions on Communications, vol. 57, no. 11, pp. 3489-3499, 2009.

[97] D. N. C. Tse and P. Viswanath, "Fundamentals of wireless communications." Cambridge University Press, 2005.

[98] H. Q. Ngo, E. Larsson, and T. Marzetta, "Energy and Spectral Efficiency of Very Large Multiuser MIMO Systems," IEEE Transactions on Communications, vol. 61, no. 4, 2013.

[99] G. Golub and C. V. Loan, "Matrix computations," The Johns Hopkins University Press, 1996.

[100] J. Choi and F. Adachi, "User selection criteria for multiuser systems with optimal and suboptimal LR-based detectors," IEEE Transactions on Signal Processing, vol. 58, no. 10, pp. 5463 - 5468, October 2010. 


\section{A}

\section{Appendix A}

Let us consider the estimated symbol vector in the $n$-th LR-SIC layer given by (3-25) with $\sigma \mathbf{T}$ is replaced by a $N_{t} \times N_{t}$ matrix $\sigma_{n} \mathbf{M}$ (matrix $\mathbf{M}$ can be either $\mathbf{M}=\mathbf{C}=\sqrt{\mathbf{R}^{-1}}$ or $\left.\mathbf{M}=\mathbf{T} / \sigma_{s}\right)$. Then

$$
\tilde{\mathbf{z}}_{n}=\left[\begin{array}{c}
\tilde{\mathbf{H}}_{[n]} \\
\sigma_{n} \mathbf{M}_{[n]}
\end{array}\right]^{\dagger}\left[\begin{array}{c}
\mathbf{y}-\tilde{\mathbf{H}}_{[n r]} \hat{\mathbf{z}}_{[n r]} \\
-\sigma_{n} \mathbf{M}_{[n r]} \hat{\mathbf{z}}_{[n r]}
\end{array}\right],
$$

or

$$
\tilde{\mathbf{z}}_{[n]}=\mathbf{B}_{[n]}\left[\tilde{\mathbf{H}}_{[n]}^{\mathcal{H}}\left(\mathbf{y}-\tilde{\mathbf{H}}_{[n r]} \hat{\mathbf{z}}_{[n r]}\right)-\sigma_{n}^{2} \mathbf{M}_{[n]}^{\mathcal{H}} \mathbf{M}_{[n r]} \hat{\mathbf{z}}_{[n r]}\right],
$$

where

$$
\mathbf{B}_{[n]}=\left(\tilde{\mathbf{H}}_{[n]}^{\mathcal{H}} \tilde{\mathbf{H}}_{[n]}+\sigma_{n}^{2} \mathbf{M}_{[n]}^{\mathcal{H}} \mathbf{M}_{[n]}\right)^{-1} .
$$

Now let $\mathbf{D}_{n}$ and $\mathbf{D}_{n r}$ be matrices that remove the first $(n-1)$ and the last $\left(N_{t}-n+1\right)$ columns of $\mathbf{M}$, respectively, such that $\mathbf{M}_{[n]}=\mathbf{M D}_{n}$ and $\mathbf{M}_{[n r]}=\mathbf{M D}_{n r}$. Then the matrix products in (A-2) and (A-3) can be expressed

$$
\mathbf{M}_{[n]}^{\mathcal{H}} \mathbf{M}_{[n r]}=\mathbf{D}_{n}^{\mathcal{H}}\left(\mathbf{M}^{\mathcal{H}} \mathbf{M}\right) \mathbf{D}_{n r}
$$

and

$$
\mathbf{M}_{[n]}^{\mathcal{H}} \mathbf{M}_{[n]}=\mathbf{D}_{n}^{\mathcal{H}}\left(\mathbf{M}^{\mathcal{H}} \mathbf{M}\right) \mathbf{D}_{n} .
$$

Now, since $\mathbf{C}^{\mathcal{H}} \mathbf{C}=\mathbf{R}^{-1}=\mathbf{T}^{\mathcal{H}} \mathbf{T} / \sigma_{s}^{2}$, then from (A-4) and (A-5) we conclude that the use of $\mathbf{M}=\mathbf{T} / \sigma_{s}$ or $\mathbf{M}=\mathbf{C}$ in (A-1) will yield the same result in (A-2). 
B

\section{Appendix B}

Let $\mathbf{z}$ be the transmitted symbol vector in the $L R$ domain and $\mathbb{L}=$ $\left\{\mathbf{z}^{1}, \mathbf{z}^{2}, \ldots, \mathbf{z}^{L}\right\}$ a list of candidates generated by different detections in the LR domain (branch detectors). The final decision is made through the ML cost:

$$
\hat{\mathbf{z}}=\arg \min _{\mathbf{z}^{i} \in \mathbb{L}}\left\|\mathbf{y}-\tilde{\mathbf{H}} \mathbf{z}^{i}\right\|^{2} .
$$

Let $e$ be the error event. The error probability of the list-based detector is given by

$$
\begin{aligned}
P(e) & =P(e, \mathbf{z} \notin \mathbb{L})+P(e, \mathbf{z} \in \mathbb{L}) \\
& =P(e \mid \mathbf{z} \notin \mathbb{L}) P(\mathbf{z} \notin \mathbb{L})+P(e, \mathbf{z} \in \mathbb{L}) \\
& =P(\mathbf{z} \notin \mathbb{L})+P(e, \mathbf{z} \in \mathbb{L}) .
\end{aligned}
$$

Now, since the events $\{\mathbf{z} \notin \mathbb{L}\}$ and $\left\{\mathbf{z} \neq \mathbf{z}^{1}, \mathbf{z} \neq \mathbf{z}^{2}, \ldots, \mathbf{z} \neq \mathbf{z}^{L}\right\}$, are equal, then for all $i=1,2, \ldots, L$,

$$
P(\mathbf{z} \notin \mathbb{L}) \leqslant P\left(\mathbf{z} \neq \mathbf{z}^{i}\right)=P_{e}^{i},
$$

where $P_{e}^{i}$ is the detection error probability of branch $i$ detector. Furthermore, if $\mathbf{z} \in \mathbb{L}$ and since $\mathbb{L} \subset \tilde{\mathcal{A}}$, we have that

$$
P(e, \mathbf{z} \in \mathbb{L}) \leqslant P_{e}^{M L},
$$

where $P_{e}^{M L}$ denotes the error probability of the ML detector. It then results from (B-2), (B-3) and (B-4) that

$$
P(e) \leqslant P_{e}^{M L}+P_{e}^{i} .
$$

Now, since the ML detector reaches full diversity gain $N_{r}$, then if any of the branch detectors also reaches full diversity, so does the list-based detector. Furthermore, since LR-SIC detection was shown to have full diversity gain [100], any list-based detector that includes a LR-SIC detection reaches full diversity. An additional coding gain might be observed by obtained better candidates as verified with VLD. 
C

\section{Appendix C}

Let us consider that a linear detector according to (5-21) is applied to the equivalent received signal vector (5-18) to detect the transmitted symbol vector of the $n$-th user class as in (5-33). If we define the matrix $\overline{\mathbf{A}}_{n}=\mathbf{W}_{n} \check{\mathbf{H}}_{n}$ and the vector $\overline{\mathbf{n}}_{n}=\mathbf{W}_{n} \mathbf{n}_{n}$ we can rewrite (5-33) as

$$
\tilde{\mathbf{y}}_{n}=\overline{\mathbf{A}}_{n} \mathbf{s}_{n}+\overline{\mathbf{n}}_{n},
$$

then, in analogy with the analysis in Section IV, the sum rate for the $n$-th user class after the detection is given by

$$
R_{n}=\sum_{i=1}^{N_{t_{n}}} \log _{2}\left(1+\overline{\lambda_{i}}\right),
$$

where the values $\bar{\lambda}_{i}$ are the eigenvalues of the matrix $\overline{\mathbf{B}}_{n}^{\mathcal{H}} \overline{\mathbf{B}}_{n}$ with $\overline{\mathbf{B}}_{n}=$ $\mathbf{K}_{\overline{\mathbf{n}}_{n}}^{-1 / 2} \overline{\mathbf{A}}_{n} \mathbf{K}_{\mathbf{s}_{n}}^{1 / 2}$. Then

$$
\overline{\mathbf{B}}_{n}^{\mathcal{H}} \overline{\mathbf{B}}_{n}=\mathbf{K}_{\mathbf{s}_{n}}^{1 / 2} \overline{\mathbf{A}}_{n}^{\mathcal{H}} \mathbf{K}_{\overline{\mathbf{n}}_{n}}^{-1} \overline{\mathbf{A}}_{n} \mathbf{K}_{\mathbf{s}_{n}}^{1 / 2}
$$

where $\mathbf{K}_{\mathbf{s}_{n}}=\sigma_{s}^{2} \mathbf{I}$ and $\mathbf{K}_{\overline{\mathbf{n}}_{n}}=\mathbf{W}_{n} \mathbf{K}_{\mathbf{n}_{n}} \mathbf{W}_{n}^{\mathcal{H}}$. Assuming that $\mathbf{K}_{\overline{\mathbf{n}}_{n}}$ has an inverse, we finally obtain

$$
\begin{aligned}
\overline{\mathbf{B}}_{n}^{\mathcal{H}} \overline{\mathbf{B}}_{n} & =\sigma_{s}^{2} \overline{\mathbf{A}}_{n}^{\mathcal{H}}\left(\mathbf{W}_{n} \mathbf{K}_{\mathbf{n}_{n}} \mathbf{W}_{n}^{\mathcal{H}}\right)^{-1} \overline{\mathbf{A}}_{n} \\
& =\sigma_{s}^{2} \check{\mathbf{H}}_{n}^{\mathcal{H}} \mathbf{W}_{n}^{\mathcal{H}}\left(\mathbf{W}_{n} \mathbf{K}_{\mathbf{n}_{n}} \mathbf{W}_{n}^{\mathcal{H}}\right)^{-1} \mathbf{W}_{n} \check{\mathbf{H}}_{n} \\
& =\sigma_{s}^{2} \check{\mathbf{H}}_{n}^{\mathcal{H}} \mathbf{K}_{\mathbf{n}_{n}}^{-1} \check{\mathbf{H}}_{n} .
\end{aligned}
$$

Note that (C-4) and (5-31) will yield the same result which proves that the sum rate for the DSD algorithm is independent of the linear detection procedure. 